\title{
Exploring Effects in Tippers at Island Magnetic Observatories due to Realistic Depth- and Time- Varying Oceanic Electrical Conductivity
}

Rafael Rigaud ( $\nabla$ rafael.rigaud@hotmail.com )

Observatorio Nacional https://orcid.org/0000-0002-2299-6430

Mikhail Kruglyakov

Institute of Geophysics, ETH Zürich

Alexey Kuvshinov

Eidgenossische Technische Hochschule Departement Umweltsystemwissenschaften

Katia Pinheiro

Observatorio Nacional

Johannes Petereit

GFZ German Research Centre for Geosciences

Juergen Matzka

GFZ German Research Centre for Geosciences

Elena Marshalko

Eidgenossische Technische Hochschule Departement Umweltsystemwissenschaften

Full paper

Keywords: Ocean induction effect, tippers, 3-D electromagnetic modeling, island geomagnetic observatories, oceanic 32 electrical conductivity

Posted Date: May 8th, 2020

DOI: https://doi.org/10.21203/rs.3.rs-26496/v1

License: (a) (1) This work is licensed under a Creative Commons Attribution 4.0 International License. Read Full License 
${ }_{1}$ Exploring Effects in Tippers at Island Magnetic Observatories 2 due to Realistic Depth- and Time-Varying Oceanic Electrical

\section{${ }_{3}$ Conductivity}

${ }_{4}$ Rafael Rigaud $^{1 *}$, Mikhail Kruglyakov ${ }^{2,3}$, Alexey Kuvshinov², Katia Pinheiro ${ }^{1}$, Johannes Petereit $^{4}$, Juergen Matzka ${ }^{4}$ and Elena Marshalko ${ }^{2,5,6}$

*Correspondence: rafael.rigaud@hotmail.com 


\section{Abstract}

7 Geomagnetic observatories around the world continuously measure time variations of the geomagnetic

8 field. Long-period ( $>3$ hours) variations are traditionally used to constrain the electrical conductivity

9 of the Earth's mantle either in terms of one-dimensional (1-D) or three-dimensional (3-D) conductivity

distributions. Recent studies have also shown that vertical transfer functions (tippers) estimated from

short-period ( $<3$ hours) variations at island observatories can constrain the 1-D conductivity

distribution of the oceanic lithosphere and upper mantle. This is feasible due to the

bathymetry-dependent ocean induction effect (OIE), which originates from lateral conductivity

contrasts between ocean and land and leads to non-zero tippers even for 1-D conductivity distributions

below the ocean. Thus, proper analysis of island tippers requires accurate 3 -D modeling of the OIE, for

which so far was performed assuming constant sea water electric conductivity with depth. However,

significant changes of electric conductivity with depth in the top hundreds of meters of the water

column do occur. In this study we explore - using rigorous 3-D electromagnetic (EM) modeling - to

what extent realistic, depth-dependent, oceanic conductivity affects island tippers. The modeling is performed for 10 island observatories around the world in the period range $10^{-1}$ to $10^{4}$ seconds, for

which a perceptible OIE on tippers is expected. We then compare the predicted tippers with tippers estimated from the observatory data. We also investigate, again using 3-D EM modeling, the effect of seasonal variations of the oceanic conductivity and to which extent this could explain the observed systematic seasonal variation of tippers. We find good agreement between predicted and estimated tippers for all 10 island observatories. This confirms that tippers at island observatories are mainly influenced by the bathymetry-dependent OIE. Our model studies suggest that for most of the considered island observatories, the effect from depth-varying oceanic conductivity is tangible and exceeds the error floor of 0.025 , which usually is assigned to tippers during their inversion. Contrarily, the effects

from seasonally varying oceanic conductivity were found to be too small to be worth consideration.

\section{Keywords}

Ocean induction effect, tippers, 3-D electromagnetic modeling, island geomagnetic observatories, oceanic electrical conductivity 
${ }_{34}$ One of the geophysical methods to probe the physical parameters of the Earth's mantle is Geomagnetic 35 Depth Sounding (GDS; Banks, 1969; Weidelt, 1972). GDS exploits magnetic field variations of magneto${ }_{36}$ spheric and/or ionospheric origin and allows to constrain electrical conductivity at depth. The main data ${ }_{37}$ source for GDS are magnetic field measurements performed at the global net of geomagnetic observato${ }_{38}$ ries. Long-period ( $>3$ hours) variations are routinely used in GDS to constrain electrical conductivity 39 of the Earth's mantle either in terms of local one-dimensional (i.e. vertical, 1-D; Olsen, 1998; Utada 40 et al, 2003; Munch et al, 2018, among others) or three-dimensional (3-D; Kelbert et al, 2009; Semenov ${ }_{41}$ and Kuvshinov, 2012; Koyama et al, 2014; Sun et al, 2015, among others) conductivity distributions. 42 Recent studies (Samrock and Kuvshinov, 2013; Morschhauser et al, 2019) have also shown that vertical 43 transfer functions (tippers) estimated from short-period ( $<3$ hours) variations at island observatories 44 can be used to constrain 1-D conductivity distributions of the oceanic lithosphere and upper mantle.

${ }_{45}$ This becomes feasible due to the ocean induction effect (OIE; cf. Parkinson and Jones, 1979), which 46 originates from the lateral conductivity contrast between ocean and land. The OIE leads to non-zero ${ }_{47}$ tippers even for 1-D conductivity distribution beneath the ocean (cf. Samrock and Kuvshinov, 2013).

${ }_{48}$ The interpretation of island tippers requires accurate 3-D electromagnetic (EM) modeling of the OIE 49 that takes into account the bathymetry around the observatory. So far, these models assumed constant 50 oceanic electric conductivity with depth (cf. Samrock and Kuvshinov, 2013; Morschhauser et al, 2019). ${ }_{51}$ In many regions of the world, however, the oceanic conductivity varies significantly within the uppermost ${ }_{52}$ few hundred meters of the water column (cf. Tyler et al, 2017). In this study, we explore the extent to ${ }_{53}$ which realistic depth-varying oceanic conductivity affects island tippers. Our analysis is performed for ${ }_{54} 10$ island observatories located in the Pacific, Atlantic and Indian Ocean (cf. Figure 1). In addition, we ${ }_{55}$ investigate the effect of seasonal variations of oceanic conductivity and to which extent this could explain 56 the systematic seasonal variations in tippers showed by Araya Vargas and Ritter (2016) and attributed 57 to the variability of external magnetic source fields.

\section{${ }_{58}$ Methods}

\section{Tippers}

${ }_{60}$ In non-polar regions, the source of the magnetic field variations with periods shorter than 3 hours is ${ }_{61}$ well approximated by a vertically incidenting plane wave. The plane wave assumption allows one to 
62

relate the vertical component $B_{z}$ with the horizontal component $\mathbf{B}_{H}=\left(B_{x} B_{y}\right)$ via the so-called tipper $\mathbf{T}=\left(T_{z x} T_{z y}\right)($ Berdichevsky and Dmitriev, 2008)

$$
B_{z}(\omega, \mathbf{r})=T_{z x}(\omega, \mathbf{r}) B_{x}(\omega, \mathbf{r})+T_{z y}(\omega, \mathbf{r}) B_{y}(\omega, \mathbf{r})
$$

where $\omega=2 \pi / P$ is the angular frequency of magnetic field variations with period $P$. The $x-$ and $y$-directions are defined in this paper as the directions to geographic North and East, respectively, and $z$ is vertically downwards. As a consequence of the plane-wave excitation, $B_{z}$ (and thus $\mathbf{T}$ ) are nonzero only above non-1-D conductivity structures. In fact, one can interpret $\mathbf{T}$ as a measure of the tipping of the magnetic field out of the horizontal plane above 2-D or/and 3-D conductivity structures.

\section{Global oceanic conductivity model}

The global oceanic electric conductivity model used here is that by Petereit et al (2019), which is based on the Coriolis Oceanographic data set for Re-Analysis (CORA5.0, Cabanes et al, 2013) provided by the Copernicus Marine Environment Monitoring Service. The model consists of monthly 3-D oceanic electrical conductivity distributions for 1990 to 2016. The conductivity is computed on a lateral grid of $0.5^{\circ} \times 0.5^{\circ}$ resolution and at 152 vertical levels between the sea surface and $2000 \mathrm{~m}$. More details on the oceanographic data set and the calculation of the global oceanic conductivity model can be found in Petereit et al (2019). From $2000 \mathrm{~m}$ to the ocean bottom the sea water conductivity was set to 3.2 S/m (cf. Tyler et al, 2017). As an example, Figure 2 presents global maps of the depth-averaged oceanic conductivity for a number of depth intervals for December 2015, illustrating the fact that the oceanic conductivity indeed varies with depths and laterally.

\section{Constructing 3-D conductivity models around island observatories}

The (Cartesian) 3-D conductivity models were constructed separately for each observatory. The models include a nonuniform oceanic bathymetry and a landmass with a uniform conductivity of $0.01 \mathrm{~S} / \mathrm{m}$. The latter is defined here as the upper crust from the surface down to the maximum depth of the ocean in the considered region. The landmass and the ocean comprise the 3-D part of the model, which is underlain by a crust and mantle with the 1-D conductivity distribution (cf. Figure 3) by Grayver et al (2017). Their 1-D model was obtained by joint inversion of satellite-detected tidal and magnetospheric signals and is believed to represent the globally averaged 1-D conductivity profile beneath the oceans. More details on our models are as follows. First, we note that as far as we exploit integral equation (IE) approach to calculate the EM fields (to be discussed in the next section), the modeling domain is confined to the 3-D 
part of the model. The vertical size of the domain corresponds to the maximum depth of the ocean in the region of interest, elevations above sea level are ignored. The lateral cross-section of the 3-D volume is a square of $356 \times 356 \mathrm{~km}^{2}$ with the island observatory in its center. The 3-D part of the models is constructed using bathymetry data from the National Oceanic and Atmospheric Administration (NOAA) ETOPO1 Global Relief Model (Amante and Eakins, 2009), which is provided in spherical coordinates with a lateral resolution of $1 \mathrm{arc} \min$, corresponding to $1.86 \mathrm{~km}$ at the equator. Note that in the vicinity of the GAN observatory the NOAA bathymetry model appeared to be not accurate enough, and we used data from the General Bathymetry Chart of the Oceans (GEBCO, 2019), which has a 15 arc sec horizontal resolution $(0.46 \mathrm{~km}$ at the equator). Bathymetry was converted to Cartesian coordinates by the use of the Transverse Mercator map projection and then linearly interpolated to a regular grid with $1 \mathrm{~km}$ horizontal resolution.

The uppermost left panel in Figures 5-14 shows the bathymetry in the vicinity of the considered island observatory. Vertically, the 3-D modeling domain was discretized by ten $100 \mathrm{~m}$ thick layers from the surface down to $1000 \mathrm{~m}$ depth; from $1000 \mathrm{~m}$ down to the maximum bathymetry in the region, the domain was discretized by $500 \mathrm{~m}$ thick layers (see Figure 4). Oceanic conductivity within each vertical layer is assumed to be constant and is set to the 3-D average value of the 2015 December (or June) oceanic conductivity model for the layer's volume. The models from 2015 year are chosen since most of experimental tippers are derived from the data for this year (or adjacent years).

Outside the 3-D volume, at depths between the surface and maximum depth of the ocean, conductivity is assumed to be laterally-uniform (1-D) and is set to the same oceanic conductivity profile as inside the volume, underlain by the 1-D model for crust and mantle. The uppermost right panel in Figures 5-14 depicts the vertical profile of oceanic conductivity (in purple), calculated by horizontally averaging oceanic conductivity (Figure 2) in a $5^{o} \times 5^{\circ}$ region centered at the considered island observatory. It shows that the oceanic conductivity varies significantly with depth down to approximately $1 \mathrm{~km}$ depth. Below this depth, the values are close to $3.2 \mathrm{~S} / \mathrm{m}$. This is our model for the depth-varying ocean conductivity. Furthermore, for each observatory we construct a 3-D model with a constant oceanic conductivity of $3.2 \mathrm{~S} / \mathrm{m}$ in all vertical layers. Note that such a model was used in previous studies (cf. Samrock and Kuvshinov, 2013; Morschhauser et al, 2019) to analyze island tippers. Outside the 3-D volume, the oceanic conductivity is set to $3.2 \mathrm{~S} / \mathrm{m}$, underlain by the 1 -D model for crust and mantle. This is our model for the depth-constant ocean conductivity. 


\section{3-D EM modeling}

To compute magnetic fields in 3-D conductivity models, we solve Maxwell's equations numerically, which, in the frequency domain, are written as

$$
\frac{1}{\mu_{0}} \boldsymbol{\nabla} \times \mathbf{B}(\omega, \mathbf{r})=\sigma(\mathbf{r}) \mathbf{E}(\omega, \mathbf{r})+\mathbf{j}^{e x t}(\omega, \mathbf{r})
$$

$$
\nabla \times \mathbf{E}(\omega, \mathbf{r})=-i \omega \mathbf{B}(\omega, \mathbf{r})
$$

where $\mathbf{E}(\omega, \mathbf{r}), \mathbf{B}(\omega, \mathbf{r})$ and $\mathbf{j}^{\text {ext }}(\omega, \mathbf{r})$ are the position- and frequency-dependent electric and magnetic fields and extraneous current density, respectively, $\mathbf{r}=(x, y, z), \mu_{0}$ the magnetic permeability of free space, and $\sigma$ the conductivity of the medium where the EM field propagates. Displacement currents are ignored in the considered period range.

We compute magnetic fields using the 3-D EM forward modelling solver PGIEM2G (Kruglyakov and Kuvshinov, 2018), which is based on a volume integral equation method with contracting kernel (Pankratov et al, 1995; Singer, 1995). PGIEM2G supports massive parallelization and the use of a high-order polynomial basis. However, in this study we exploit a piece-wise representation of the fields for the sake of computational efficiency. We compute magnetic fields and then tippers at 25 periods spanning the range from 0.1 to $10^{4}$ seconds.

\section{Estimating observed responses}

We estimated tippers at eight INTERMAGNET island geomagnetic observatories using definite minutemean data (time series of three components of magnetic field). In addition, we estimated tippers at two non-INTERMAGNET island observatories: St. Helena (SHE) and Santa-Maria/Azores (SMA), also using minute-mean data. Prior to the tipper estimation, obvious spikes were removed from the corresponding time series. Table 1 summarizes information about the observatories, including the time interval used for the tipper estimation. This interval varies from observatory to observatory and was selected - based on a trial and error approach - to obtain smooth (with respect to period) tippers with low uncertainties. For each period, data were split into overlapping tapered windows of two periods length. Data in these windows were Fourier transformed, giving the spectrum of the corresponding component from 300 to 9600 seconds. Tippers and their uncertainties were then estimated using a robust, section-averaging (Olsen, 1998) linear regression scheme based on the Huber norm (e.g. Aster et al, 2005; Püthe and Kuvshinov, 2014). 


\section{Results}

\section{Effect of depth-varying oceanic conductivity}

Modeling results for all 10 island geomagnetic observatories are presented in Figures 5 to 14 . In all figures, panel (a) shows the bathymetry map with the corresponding observatory located in the centre. The dashed line indicates the west-east running bathymetry profile shown in panel (b). Panel (c) shows the regional depth-varying oceanic conductivity down to $2000 \mathrm{~m}$ (purple solid line) and the constant reference oceanic conductivity $(3.2 \mathrm{~S} / \mathrm{m}$, dashed orange line). Panels (d) to (g) present the $\operatorname{Re} T_{z x}, \operatorname{Re} T_{z y}, \operatorname{Im} T_{z x}$ and $\operatorname{Im} T_{z y}$ tipper components, respectively. Red and orange curves correspond to tippers calculated in the models with depth-varying and depth-constant oceanic electrical conductivity, respectively. The blue curves represent observed tippers and their uncertainties estimated from the data. One can see that both observed and modeled tippers fulfill the property (Marcuello et al, 2005) that is often used as a plausibility check for estimated (observed) and predicted (modeled) tippers, namely, at periods where the real part of the tipper components $T_{z x}$ or $T_{z y}$ reaches a maximum (or a minimum) value, the corresponding imaginary part changes sign.

Three observations can be made immediately from panels $(\mathrm{d})-(\mathrm{g})$ that are independent of modelling being done with depth-varying or depth-constant oceanic conductivity:

(1) The manifestation of the OIE in the modeled tippers varies from observatory to observatory. This variability of the OIE is expected to be due to the different bathymetry distributions around the islands.

(2) Modeled and experimental tippers agree well for all observatories, for the full spectrum from 300 to 9600 seconds, for both components and for both the real and imaginary part. This, in particular, means that the OIE is the main player making island tippers non-zero. Any remaining discrepancy can most probably be attributed to regional deviations of the crustal and mantle conductivity structure from the global 1-D conductivity structure used for modeling.

(3) The modeled OIE in tippers can be traced to periods as short as 0.2 seconds. Here, we consider the OIE as significant if it exceeds a value of 0.025 , which is traditionally used as an error floor in tipper inversions. (e.g. Morschhauser et al, 2019; Yang et al, 2015; Tietze and Ritter, 2013; Rao et al, 2014; Bedrosian and Feucht, 2014). For HON and SMA we find $\operatorname{Im} T_{z x}$ to exceed 0.025 at 0.2 seconds. The maximum amplitude of the OIE is observed at HON observatory (cf. Figure 9), where it reaches the value of 1.1 in the real part of $T_{z x}$ at a period of $20 \mathrm{sec}$.

Figures 5-14, panels (h) and (i) show the effect of depth-varying oceanic conductivity on island tippers. 
This effect is assessed by the following difference

$$
\Delta T_{z i}=\sqrt{\left(\operatorname{Re} T_{z i}^{v}-\operatorname{Re} T_{z i}^{c}\right)^{2}+\left(\operatorname{Im} T_{z i}^{v}-\operatorname{Im} T_{z i}^{c}\right)^{2}}
$$

where $i \in[x, y]$, and superscripts " $v$ " and " $c$ " correspond to the tippers calculated in the models with depth-varying and depth-constant oceanic conductivity, respectively. The horizontal dashed line depicts the threshold value of 0.025 discussed above. As seen from Figures 5-14, the effect is notable and exceeds the threshold of 0.025 for all observatories (albeit very small in ASC, IPM and SHE). At GAN and HON, the effect is considerable, reaching values close to 0.1. On the whole, the effect becomes less pronounced at the longest considered periods.

The manifestation of the depth-varying oceanic conductivity in the tipper components and the period range varies from observatory to observatory. This variability, like the variability of OIE itself, is expected from the different bathymetry distributions around the islands.

\section{Effects of time-varying oceanic conductivity}

Figure 15 presents global maps of differences between December and June (depth-averaged) oceanic conductivity in the same depth intervals as in Figure 2. As expected, the difference varies laterally, it is the largest at shallower depths (reaching 20 percent of the mean value of oceanic conductivity) and decreases with depth.

Finally, we modeled the effect of time-varying oceanic conductivity on island tippers. The effect is assessed by analyzing the difference

$$
\widehat{\Delta T_{z i}}=\sqrt{\left(\operatorname{Re} T_{z i}^{D}-\operatorname{Re} T_{z i}^{J}\right)^{2}+\left(\operatorname{Im} T_{z i}^{D}-\operatorname{Im} T_{z i}^{J}\right)^{2}}
$$

where $i \in[x, y]$ and superscripts " $D$ " and " $J$ " correspond to December and June results. For experimental tippers, December and June results stand for tippers, estimated from observatory data of corresponding 2015 months. As for modeled tippers, these results mean tippers calculated in 3-D models with depthvarying oceanic conductivity models for 2015 December and June months.

Figures 16 and 17 demonstrate $\Delta T_{z x}$ and $\Delta T_{z y}$ at ten considered observatories as filled circles on a global map. "Experimental" and "modeled" differences are colored by light and dark blue, respectively. It is clearly seen that the effect due to time-varying oceanic conductivity is negligible. 
It is interesting to note that the temporal variability of the experimental tippers increases with period, and overall it is larger in $T_{z y}$.

\section{Conclusions}

In this study we performed the first ever analysis of the effects of realistic depth- and time-varying oceanic electrical conductivity on island tippers. The analysis is based on 3-D EM modeling, which was carried out for ten island observatories located in the Pacific, Atlantic and Indian Oceans. The conductivity models specific for each observatory were constructed using bathymetry data (ETOPO or GEBCO) and a 3-D, time-dependent global model of oceanic conductivity (Petereit et al, 2019). The Cartesian EM forward solver by Kruglyakov and Kuvshinov (2018) was used for EM modeling. Modeling was performed in period range $\left(10^{-1}-10^{4} \mathrm{sec}\right)$, where tippers are expected to be perceptible due to the ocean induction effect.

The effect due to depth-varying oceanic conductivity was assessed by comparing the tippers obtained from the depth-varying and depth-constant oceanic conductivity models. Our model studies show that in most considered observatories (except ASC, IPM and SHE) the effect is tangible. It exceeds the error floor of 0.025 which is usually assigned to tippers during their 2-D or 3-D inversion and reaches large values of around 0.1 for the HON and GAN observatories. The appearance of the effect with respect to period and its strength varies from observatory to observatory. Such lateral variability of the effect is most probably due to different bathymetry distributions around the islands. Based on our modeling results, we recommend to use depth-varying oceanic conductivity when modeling island tippers, provided these data are available and trustworthy. On the contrary, the modeled effects from time-varying oceanic conductivity appeared to be too small to explain the observed seasonal variations in tippers.

\footnotetext{
List of abbreviations

EM: Electromagnetic;

1-D: one-dimensional;

2-D: two-dimensional;

3-D: three-dimensional;

OIE: Ocean Induction Effect;

NOAA: National Oceanic and Atmospheric Administration;
} 


\section{Availability of data and materials}

${ }_{250}$ The results presented in this paper rely on $1 \mathrm{~Hz}$ data collected at geomagnetic observatories. These

251 data were digitally filtered to produce one minute means that are available from the INTERMAGNET

252 data repository. 
The authors declare that they have no competing interests.

\section{Funding}

RR was supported by CNPq, Process 133345/2018-1. MK was supported by grant 20-05-00001 from the Russian Foundation for Basic Research. AK was partially supported by the European Space Agency through the Swarm DISC project. KP was supported by FAPERJ (Jovem Cientista do Nosso Estado, Process 202.748/2019). EM was supported by grant 16-17-00121 from the Russian Science Foundation. JP was supported by the German Research Foundation's priority program 1788 Dynamic Earth.

\section{References}

Amante C., Eakins B. W. (2009) Etopo1 1 arc-minute global relief model: Procedures, data sources and analysis. noaa technical memorandum nesdis ngdc-24. national geophysical data center, noaa. doi:107289/V5C8276M

Araya Vargas J., Ritter O. (2016) Source effects in mid-latitude geomagnetic transfer functions. Geophysics Journal International 204(1):606-630

Aster R., Borchers B., Thurber C. (2005) Parameter estimation and inverse problems. Elsevier Academic Press, Waltham(MA)

Banks R. J. (1969) Geomagnetic variations and the electrical conductivity of the upper mantle. Geophysics Journal International 17(5):457-487

Bedrosian P. A., Feucht D. W. (2014) Structure and tectonics of the northwestern united states from earthscope usarray magnetotelluric data. Earth Planet Sci Lett 402:275-289

Berdichevsky M. N., Dmitriev V. (2008) Models and methods of magnetotellurics. Springer, Berlin

Cabanes C., Grouazel A., von Schuckmann K., Hamon M., Turpin V., Coatanoan C. (2013) The cora dataset: Validation and diagnostics of in-situ ocean temperature and salinity measurements. Ocean Science 9(1):1-18

GEBCO (2019) Gebco compilation group (2019) gebco 2019 grid. doi:105285/836f016a-33be-6ddc-e053-6c86abc0788e

Grayver A. V., Munch F. D., Kuvshinov A. V., Khan A., Sabaka T. J., Tøffner-Clausen L. (2017) Joint inversion of satellite-detected tidal and magnetospheric signals constrains electrical conductivity and 
water content of the upper mantle and transition zone. Geophysical Research Letters 44(12):6074-6081

Kelbert A., Schultz A., Egbert G. (2009) Global electromagnetic induction constraints on transition-zone water content variations. Nature 460:1003-1007

Koyama T., Khan A., Kuvshinov A. (2014) Three-dimensional electrical conductivity structure beneath Australia from inversion of geomagnetic observatory data: evidence for lateral variations in transition-zone temperature, water content and melt. Geophys J Int 196:1330-1350, DOI 10.1093/gji/ggt455

Kruglyakov M., Kuvshinov A. (2018) Using high-order polynomial basis in 3-d em forward modeling based on volume integral equation method. Geophys J Int 213:1387-1401

Marcuello A., Queralt P., Ledo J. (2005) Applications of dispersion relations to the geomagnetic transfer function. Physics of the Earth and Planetary Interiors 150:85-91

Morschhauser A., Grayver A. V., Kuvshinov A. V., Samrock F., Matzka J. (2019) Tippers at island geomagnetic observatories constrain electrical conductivity of oceanic lithosphere and upper mantle. Earth, Planets and Space 71(1):17

Munch F. D., Grayver A. V., Kuvshinov A., Khan A. (2018) Stochastic inversion of geomagnetic observatory data including rigorous treatment of the ocean induction effects with implications for transition zone water content and thermal structure. Journal of Geophysical Research 123:31-51

Olsen N. (1998) The electrical conductivity of the mantle beneath europe derived from c-responses from 3 to 720 hr. Geophys J Int 133(2):298-308

Pankratov O., Avdeev D., Kuvshinov A. (1995) Electromagnetic field scattering in a heterogeneous earth: A solution to the forward problem. Izvestiya, Physics of the Solid Earth 31(3):201-209

Parkinson W., Jones F. W. (1979) The geomagnetic coast effect. Reviews of Geophysics and Space Physics 17(8):1999-2017

Petereit J., Saynisch-Wagner J., Irrgang C., Thomas M. (2019) Analysis of ocean-tide induced magnetic fields derived from oceanic in situ observations: Climate trends and the remarkable sensitivity of shelf regions. Journal of Geophysical Research: Oceans 124:8257-8270

Püthe C., Kuvshinov A. (2014) Mapping 3-d mantle electrical conductivity from space: a new 3-d inversion scheme based on analysis of matrix q-responses. Geophysical Journal International 197(2):768-784 
Rao C. K., Jones A. G., Moorkamp M., Weckmann U. (2014) Implications for the lithospheric geometry of the iapetus suture beneath ireland based on electrical resistivity models from deep-probing magnetotellurics. Geophys J Int 198:737759

Samrock F., Kuvshinov A. (2013) Tipper at island observatories: Can we use them to probe electrical conductivity of the earth's crust and upper mantle? Geophysical Research Letters 40:824-828

Semenov A., Kuvshinov A. (2012) Global 3-D imaging of mantle electrical conductivity based on inversion of observatory C-responses - II. Data analysis and results. 191:965-992

Singer B. (1995) Method for solution of maxwell's equations in non-uniform media. Geophysical Journal International 120:590-598

Sun J., Kelbert A., Egbert G. D. (2015) Ionospheric current source modeling and global geomagnetic induction using ground geomagnetic observatory data. J Geophys Res Solid Earth 120:67716796, DOI 10.1002/2015JB012063

Tietze K., Ritter O. (2013) Three-dimensional magnetotelluric inversion in practicethe electrical conductivity structure of the san andreas fault in central california. Geophys J Int 195:130147

Tyler R. H., Boyer T. P., Minami T., Zweng M. M., Reagan J. R. (2017) Electrical conductivity of the global ocean. Earth, Planets and Space 69:156-166

Utada H., Koyama T., Shimizu H., Chave A. D. (2003) A semi-global reference model for electrical conductivity in the mid-mantle beneath the north pacific region. Geophysical Research Letters 30(4):1194-1198

Weidelt P. (1972) The inverse problem of geomagnetic induction, z. Geophysics 38:257-289

Yang B., Egbert G. D., Kelbert A., N. M. (2015) Three-dimensional electrical resistivity of the north-central usa from earthscope long period magnetotelluric data. Earth Planet Sci Lett 422:87-93 
Table 1. : Information on the geomagnetic observatories used in this study. From left to right: IAGA code, observatory name, geographic and geomagnetic latitude and longitude, starting date of INTERMAGNET participation, and time interval used to estimate tippers. Observatories with an asterisk $(*)$ are, as of date, not participating in INTERMAGNET and data is available from GFZ Potsdam. Geomagnetic coordinates were calculated using the IGRF-12 model, epoch 2015

\begin{tabular}{|c|c|c|c|c|c|c|c|}
\hline Code & Name & $l a t^{G G}$ & $\operatorname{lon}{ }^{G G}$ & $l a t^{G M}$ & $\operatorname{lon}{ }^{G M}$ & $\begin{array}{c}\text { Starting date } \\
\text { (INTERMAGNET) }\end{array}$ & $\begin{array}{c}\text { Time interval } \\
\text { used for tippers' estimation }\end{array}$ \\
\hline ASC & Ascension Island & 7.95 & -14.38 & -2.77 & 57.48 & 2003 & $01 / 01 / 2014-31 / 12 / 2014$ \\
\hline CKI & Cocos-Keeling Islands & 12.10 & -96.84 & -21.56 & 168.92 & 2013 & $01 / 01 / 2015-30 / 06 / 2015$ \\
\hline GAN & Gan & 0.69 & 73.15 & -8.64 & 145.33 & 2013 & $01 / 01 / 2017-31 / 12 / 2017$ \\
\hline GUA & Guam & 13.59 & 144.87 & 5.8 & 216.51 & 1991 & $01 / 01 / 2016-31 / 12 / 2016$ \\
\hline $\mathrm{HON}$ & Honolulu & 20.32 & -158 & 21.65 & 270.85 & 1991 & $01 / 01 / 2015-30 / 09 / 2015$ \\
\hline IPM & Easter Island & -27.2 & -109.42 & -19.17 & 325.61 & 2010 & $01 / 01 / 2013-31 / 12 / 2013$ \\
\hline PPT & Pamatai & -17.57 & -149.58 & -15.05 & 285.79 & 1991 & $01 / 01 / 2015-31 / 12 / 2015$ \\
\hline SHE & St. Helena & -15.96 & -5.75 & 11.78 & 64.24 & $2009 *$ & $01 / 01 / 2013-30 / 06 / 2013$ \\
\hline SMA & Santa Maria/Azores & 36.99 & -25.13 & 43.21 & 53.57 & $2018 *$ & $01 / 07 / 2018-31 / 10 / 2018$ \\
\hline TDC & Trista da Cunha & -37.07 & -12.31 & -31.70 & 54.76 & 2010 & $01 / 01 / 2015-30 / 09 / 2015$ \\
\hline
\end{tabular}

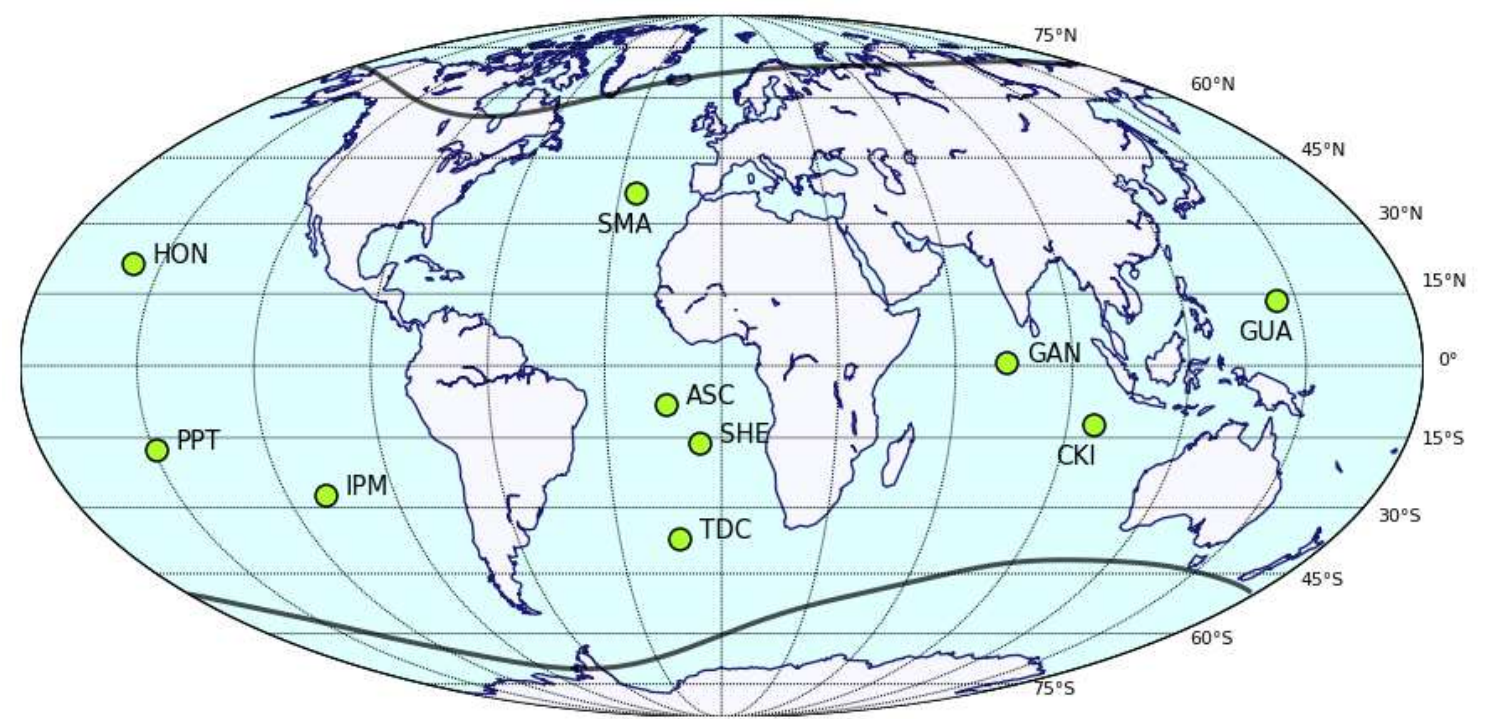

Figure 1. : Location of geomagnetic observatories used in this study (green dots). Black lines depict $\pm 55^{\circ}$ quasi dipole latitudes. Relevant information about these observatories is summarized in Table 1. 

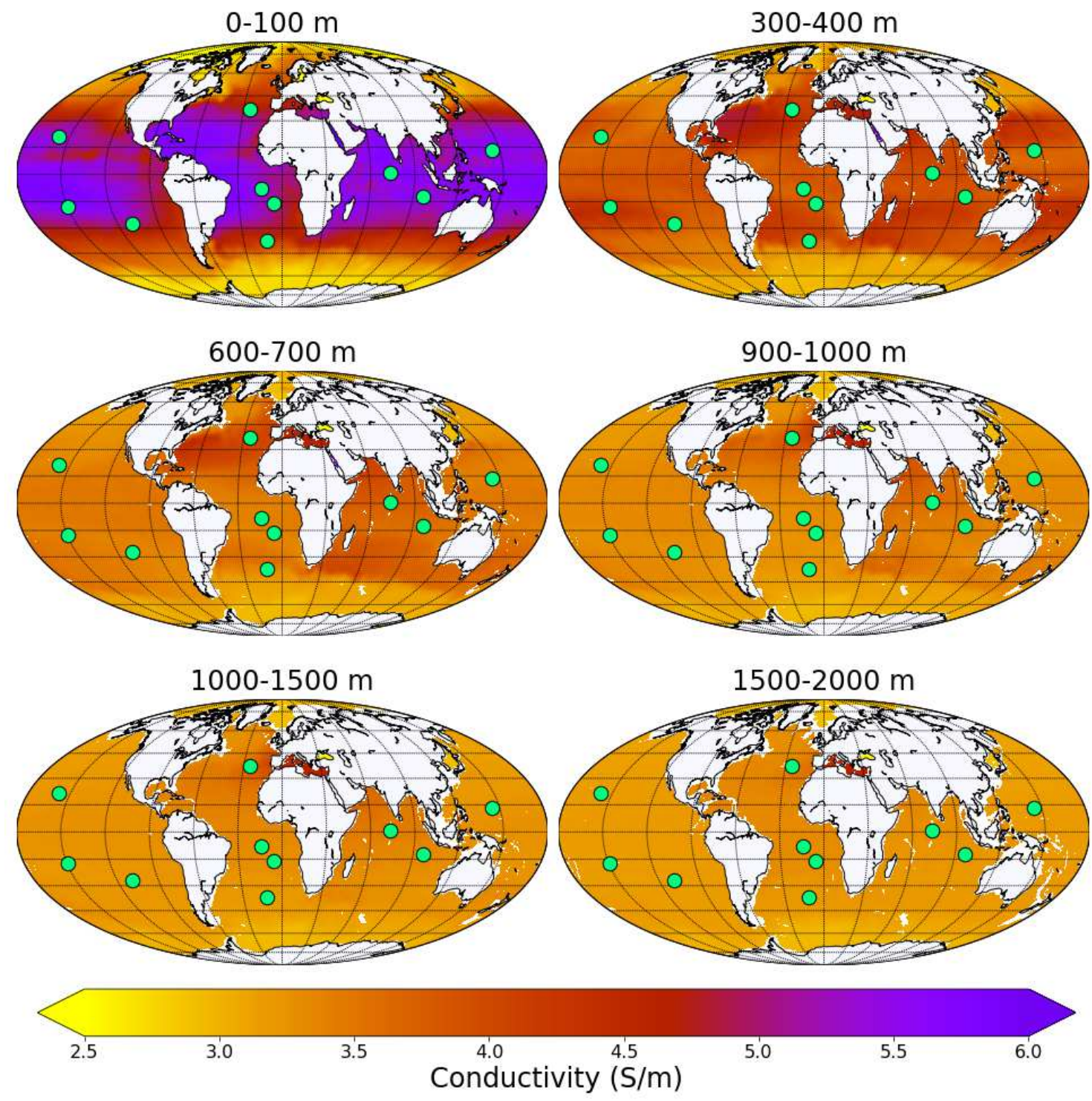

Figure 2. : Global maps of oceanic electric conductivity for six selected depth intervals for December 2015 model. Green dots denote the locations of the geomagnetic observatories used in this study. 


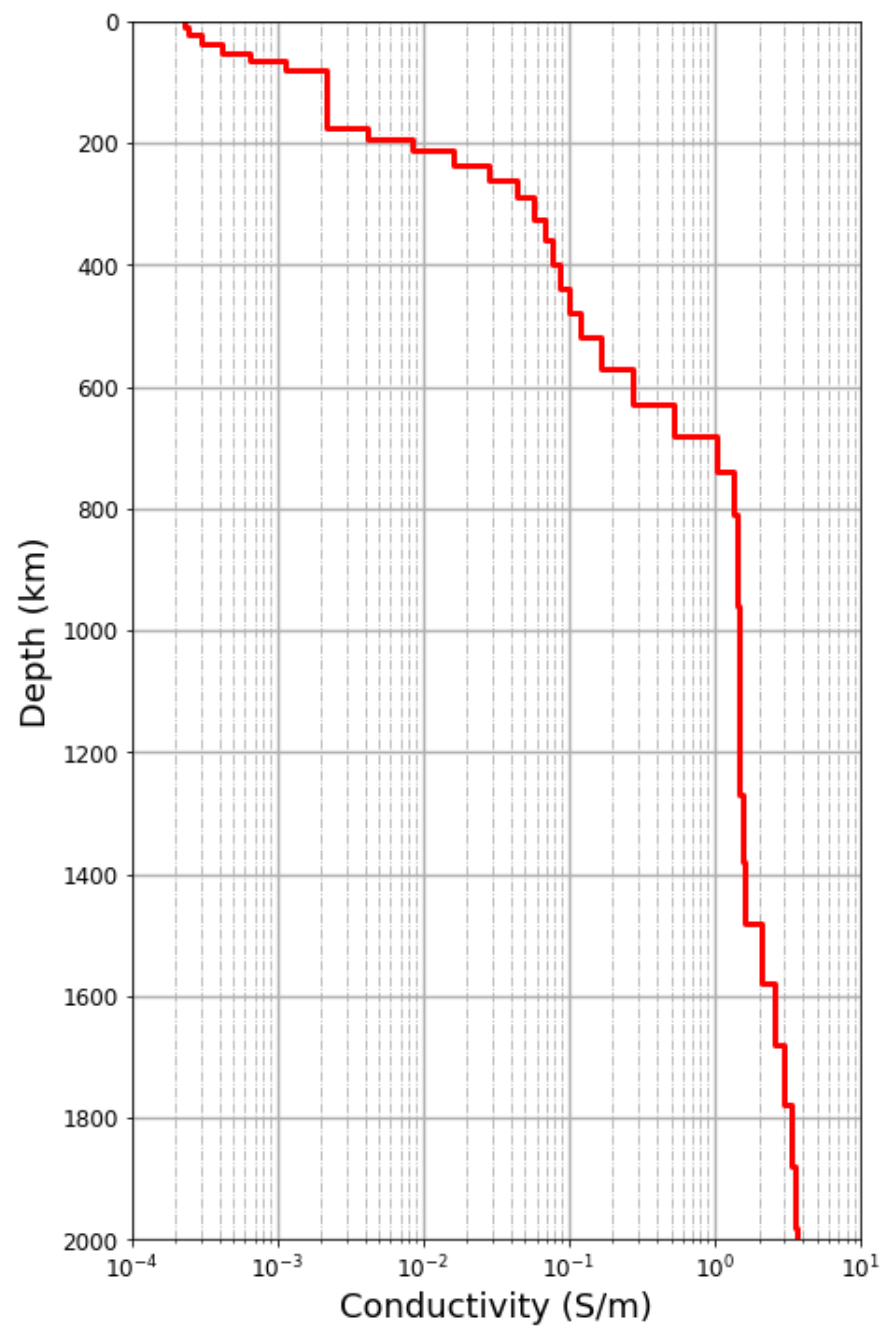

Figure 3. : 1-D global conductivity profile (from Grayver et al, 2017) used in this study for the crust and mantle beneath the 3-D (oceanic) modeling domain. 


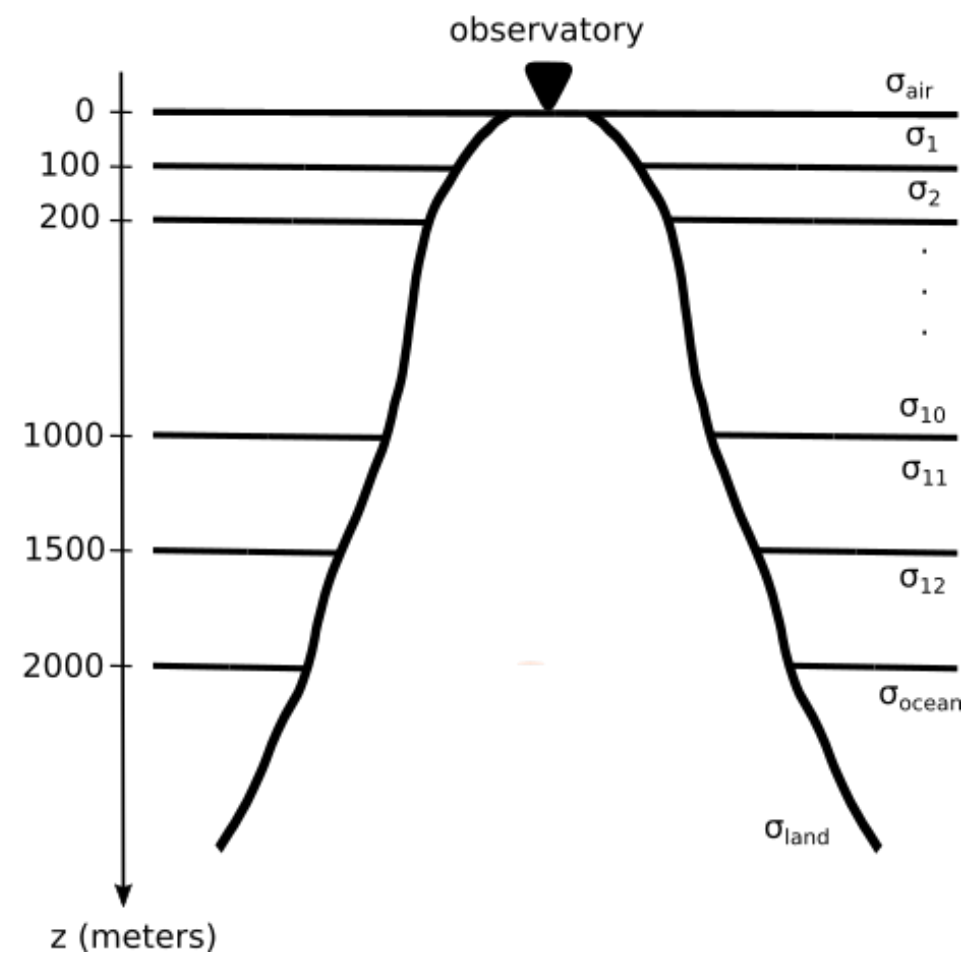

Figure 4. : Vertical parametrization of the conductivity distribution in the 3-D modelling domain. $\sigma_{1}$ to $\sigma_{12}$ is the respective water layer's conductivity, set to the layers's average conductivity calculated from the global ocean conductivity model. $\sigma_{\text {ocean }}$ is the deep (below $2000 \mathrm{~m}$ ) ocean conductivity set to $3.2 \mathrm{~S} / \mathrm{m}$. $\sigma_{\text {land }}$ is the landmass conductivity set to $0.01 \mathrm{~S} / \mathrm{m}$. 

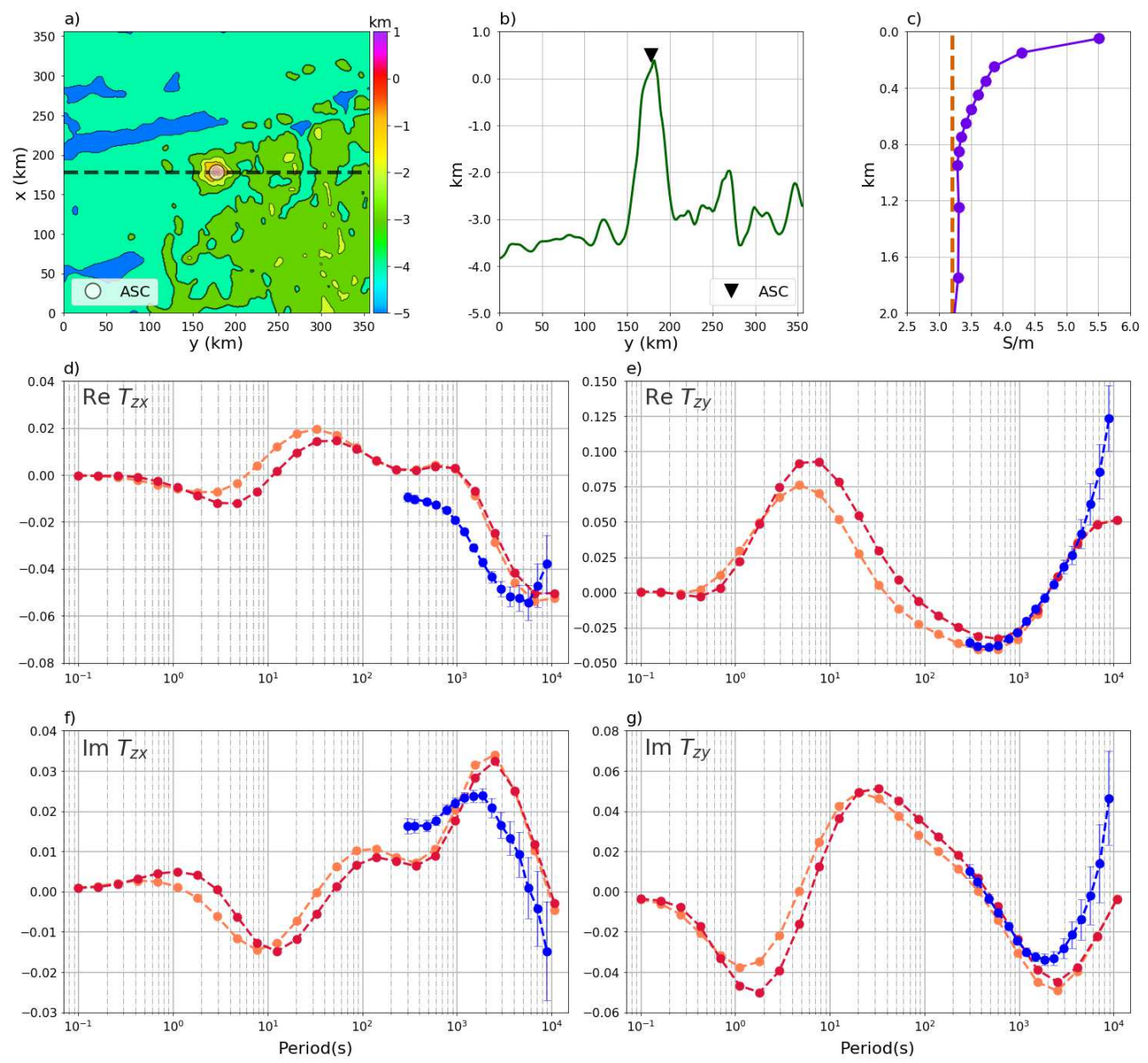

- - Modelled with constant oceanic conductivity - - - Modelled with depth-varying oceanic conductivity $\quad$ - - Observed
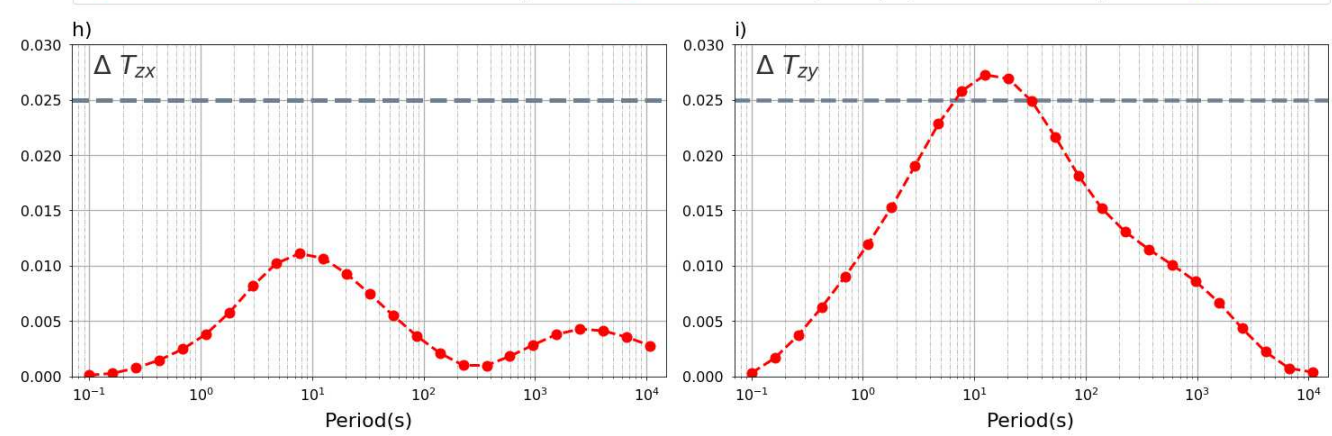

Figure 5. : Results for Ascension Island observatory (ASC). (a) Map of bathymetry; dashed line indicates location of profile shown in panel (b). (b) West-East oriented bathymetry profile. (c) Regional depth-varying (purple) oceanic conductivity and constant reference oceanic conductivity $(3.2 \mathrm{~S} / \mathrm{m}$, red dashed line). (d) to (g) Real and imaginary parts for $x$ and $y$ components of tippers computed in the model with depth-varying (orange dots and dashed line) and depth-constant (red dots and dashed line) oceanic conductivity. (h) Difference for the computed tipper $x$ component between depth-varying and depth-constant ocean conductivity, see text for details. Dashed grey line indicates the threshold of 0.025. (i) Same as (h), but for the $y$ component. 

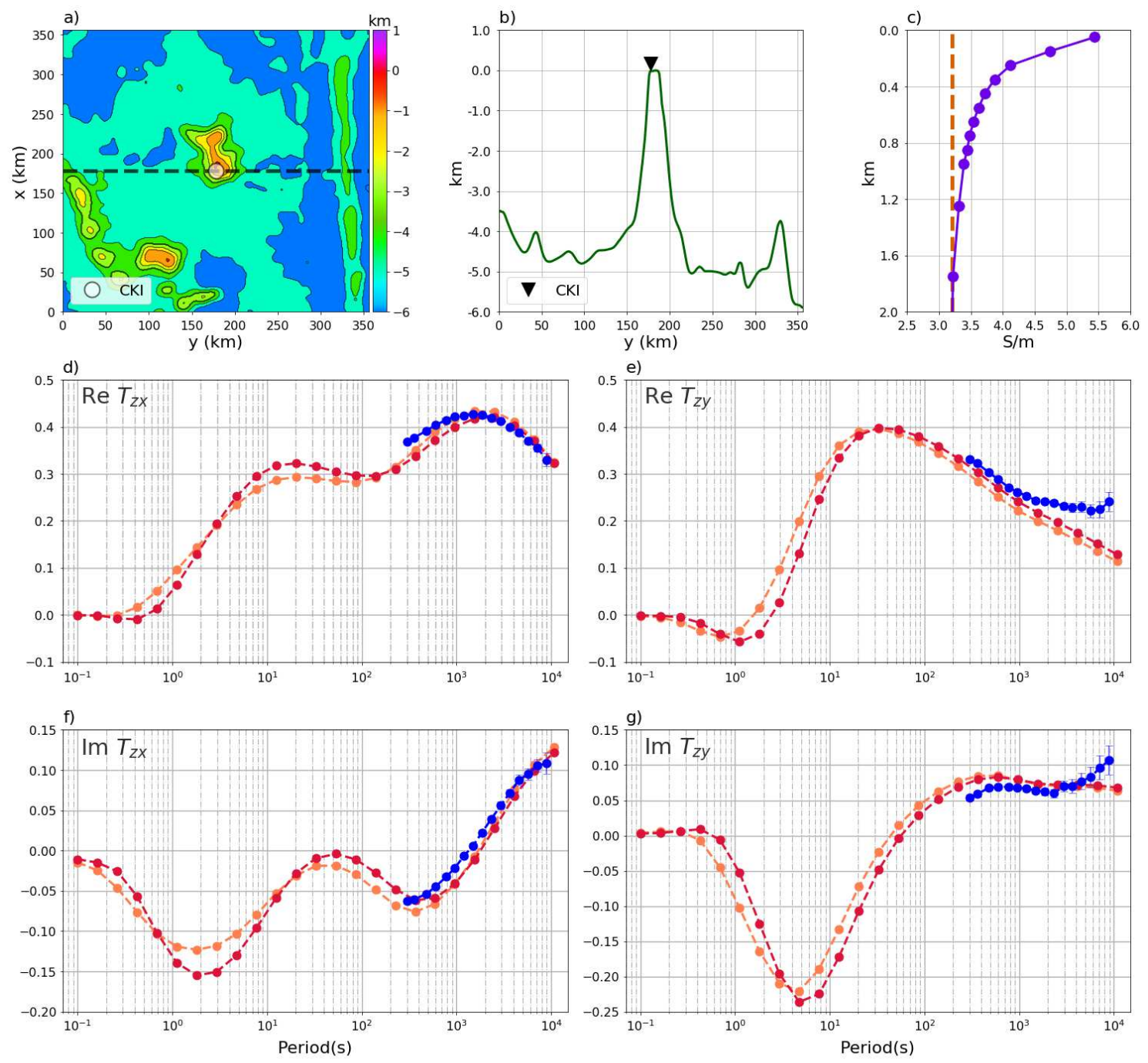

- - Modelled with constant oceanic conductivity _- - Modelled with depth-varying oceanic conductivity

- - Observed
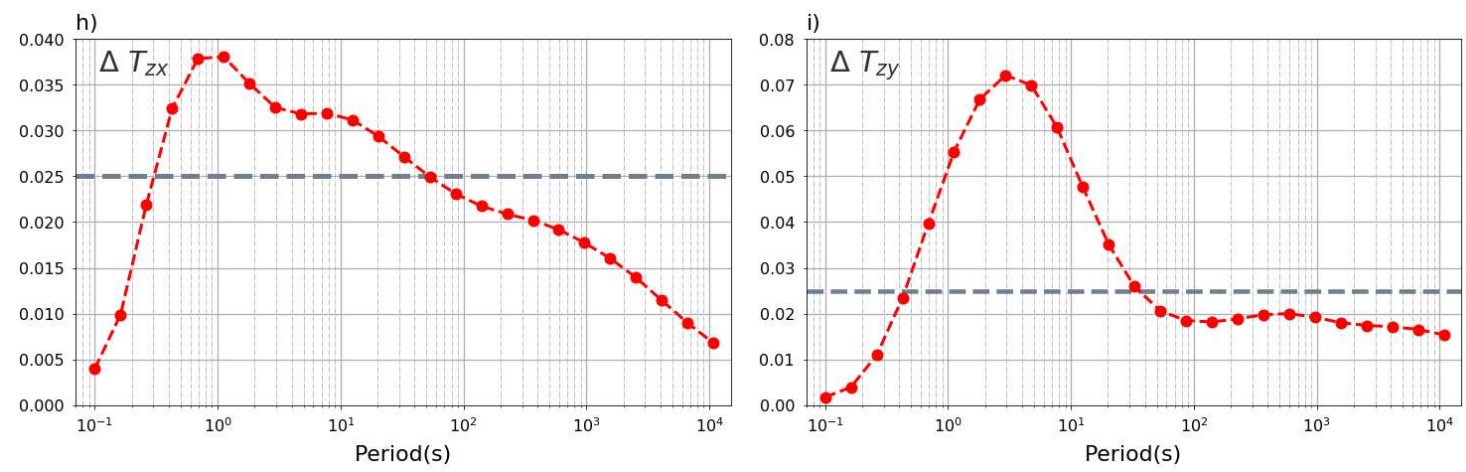

Figure 6. : Same as Figure 5, but for Cocos-Keeling Islands observatory (CKI). 

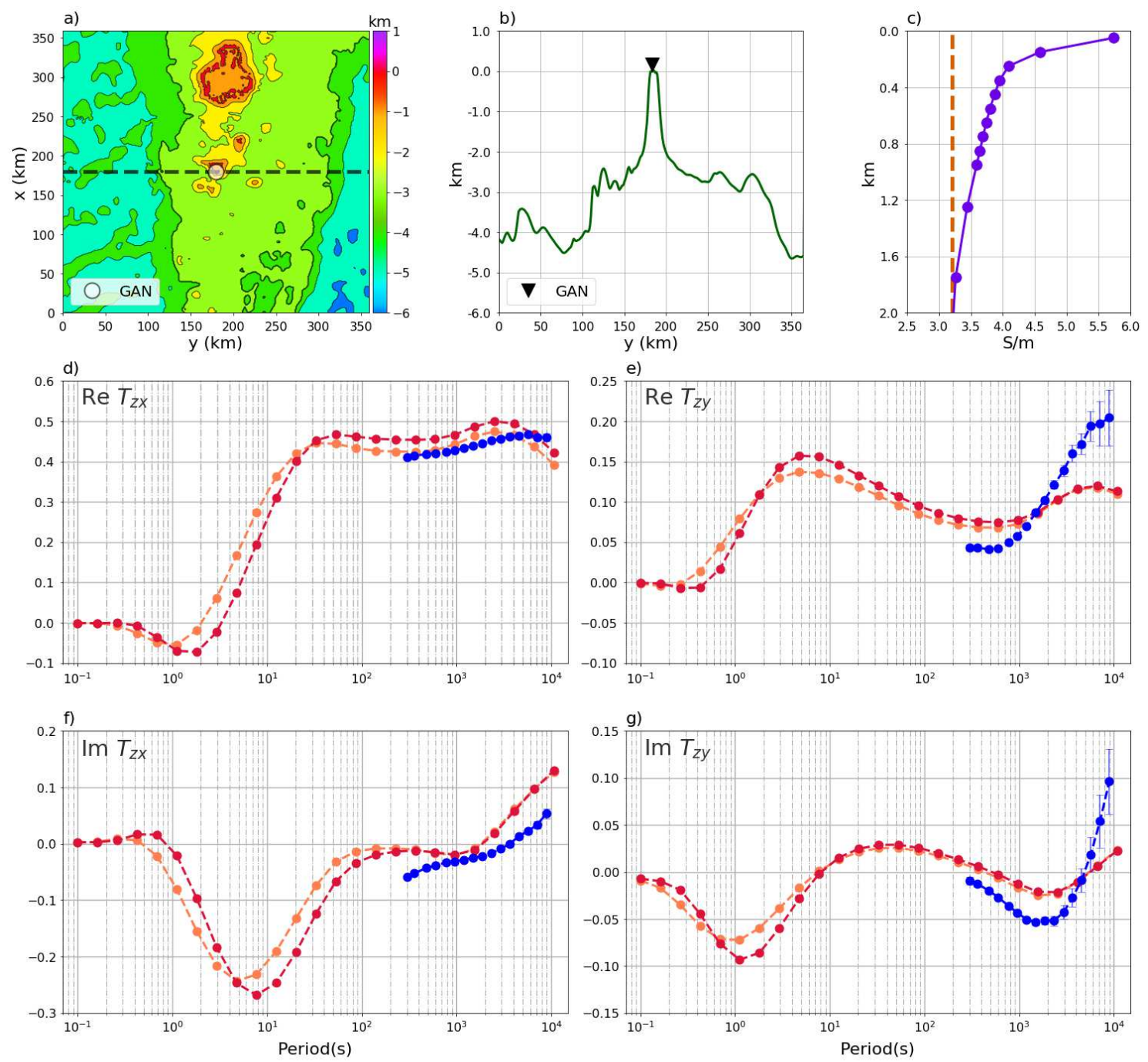

-๑ - Modelled with constant oceanic conductivity $\quad$ - - Modelled with depth-varying oceanic conductivity - - Observed
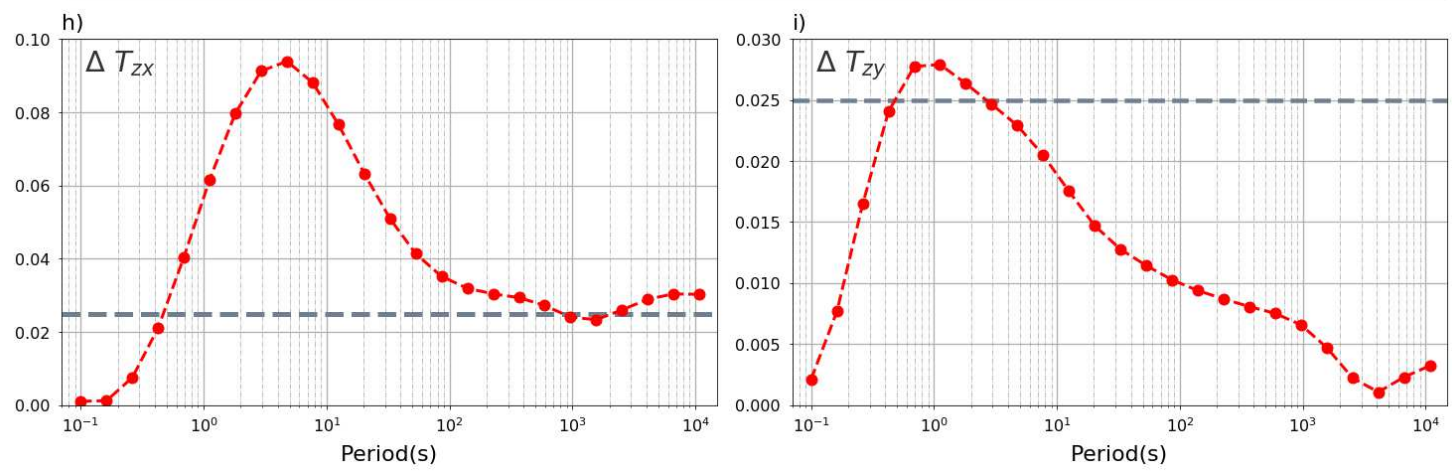

Figure 7. : Same as Figure 5, but for Gan observatory (GAN). 

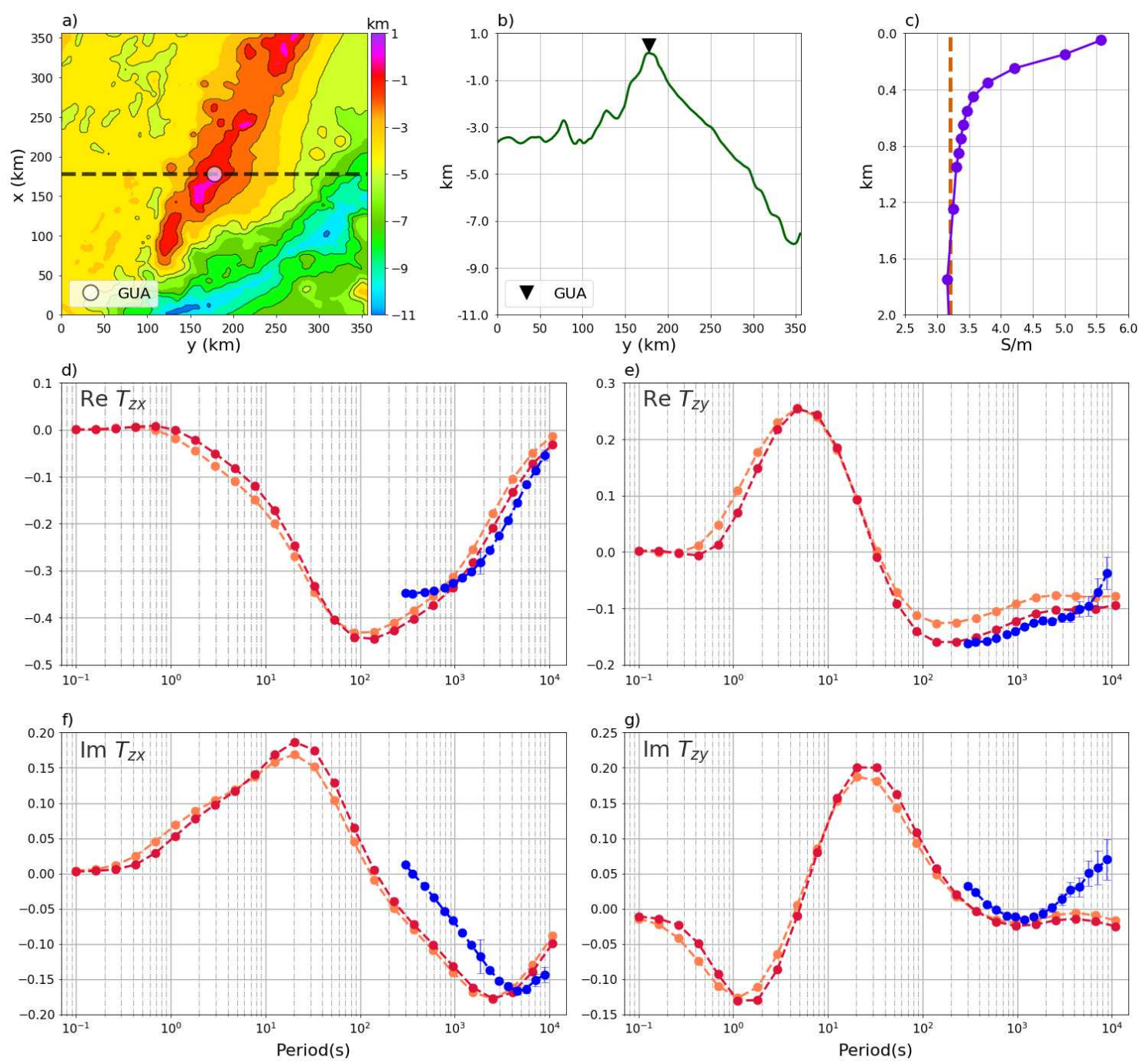

- - Modelled with constant oceanic conductivity - - - Modelled with depth-varying oceanic conductivity

- - Observed
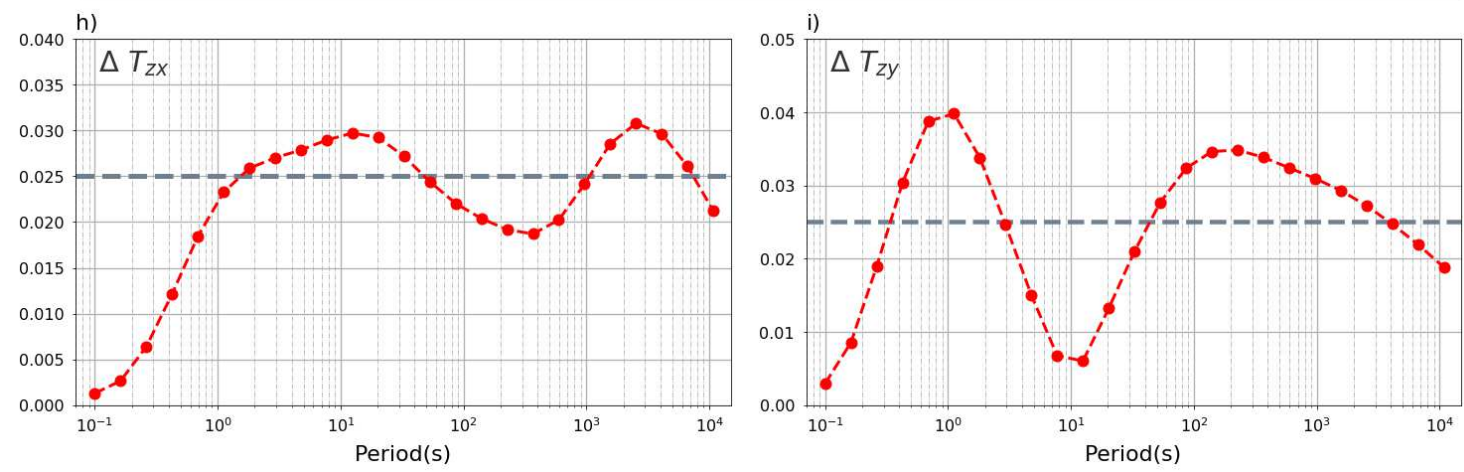

Figure 8. : Same as Figure 5, but for Guam observatory (GUA). 

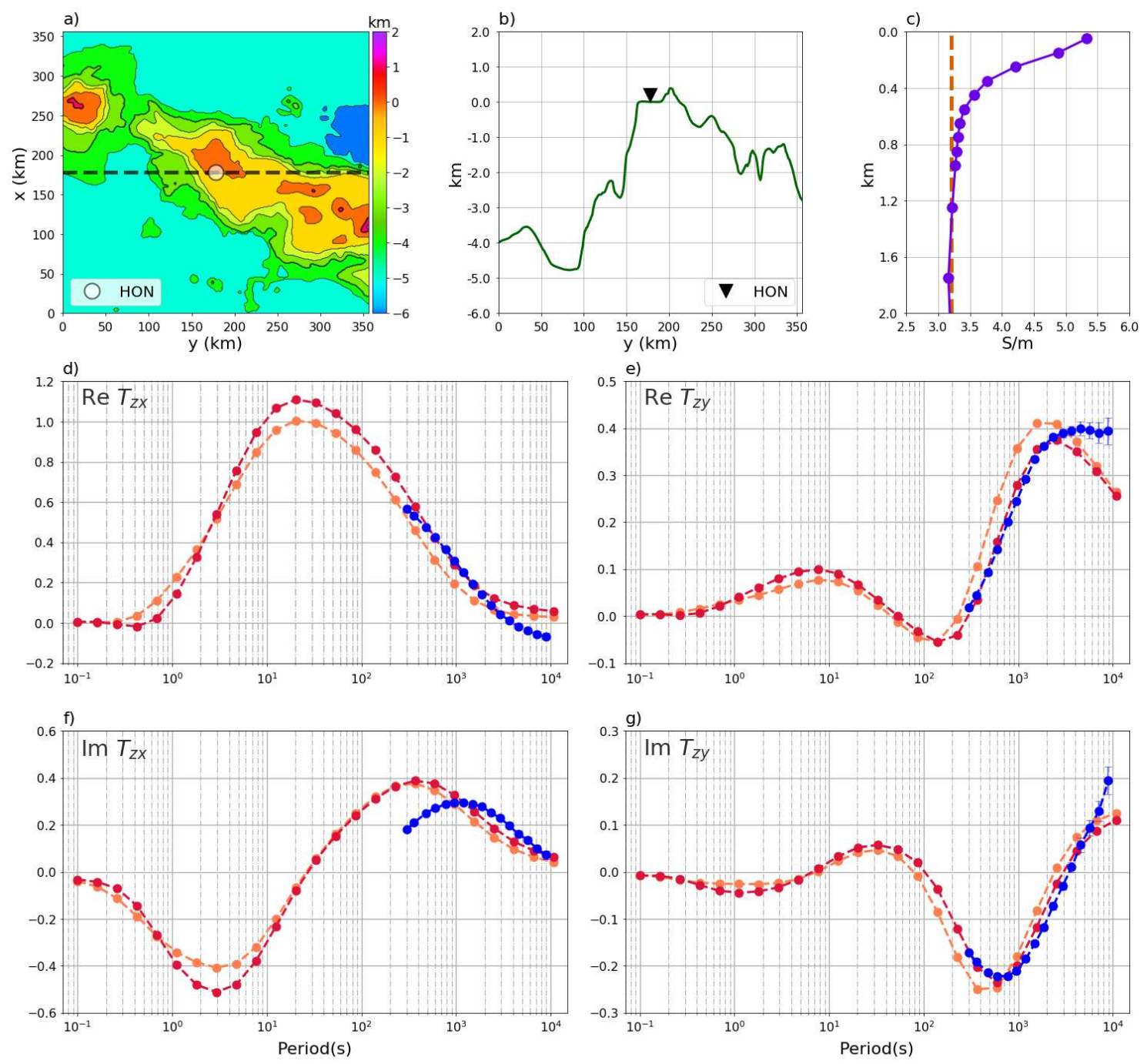

- - Modelled with constant oceanic conductivity $\quad$ - - Modelled with depth-varying oceanic conductivity

- - Observed
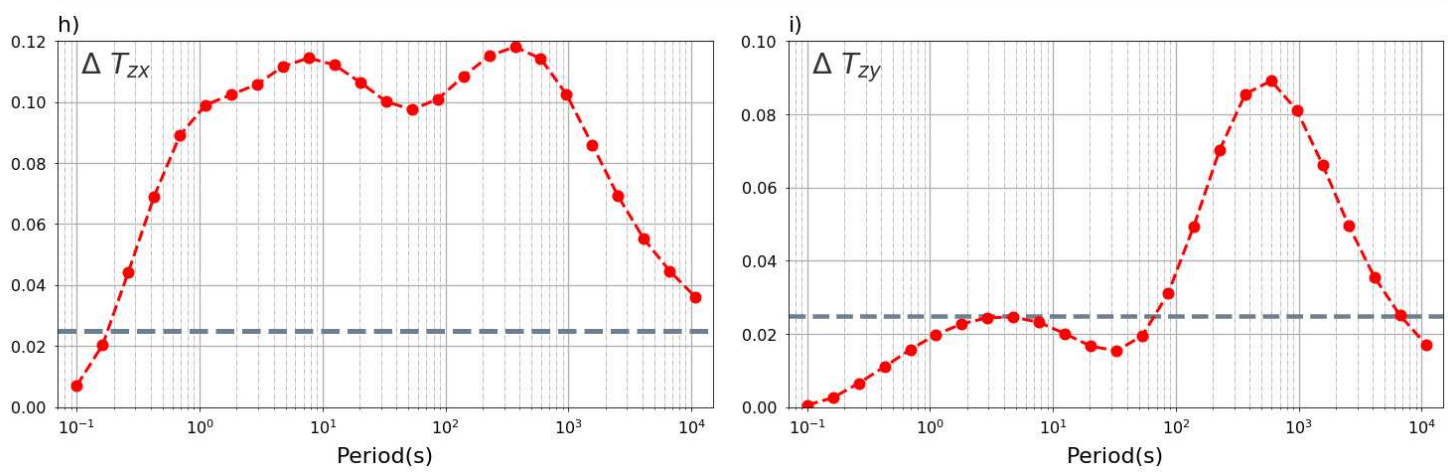

Figure 9. : Same as Figure 5, but for Honolulu observatory (HON). 

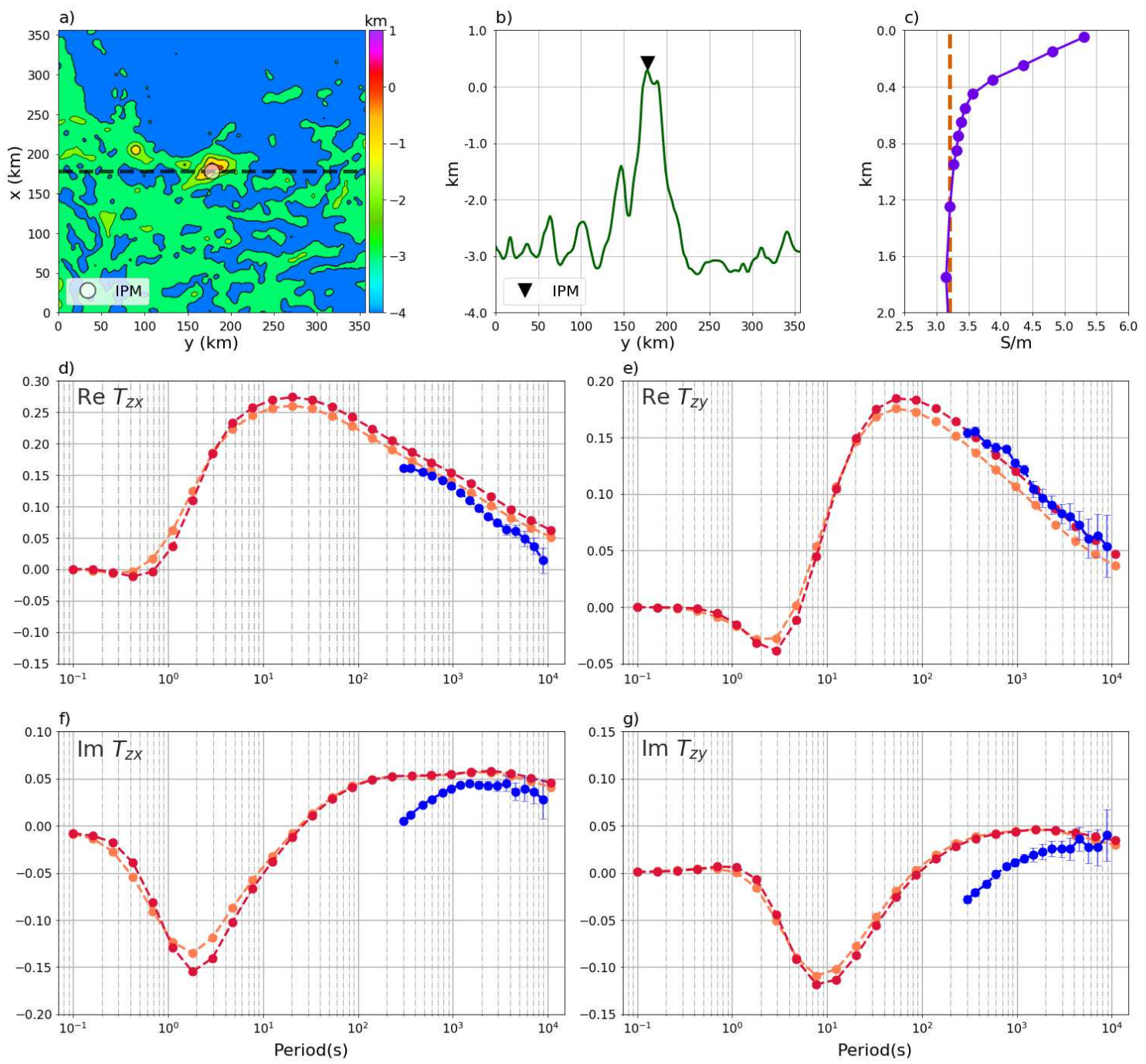

-๑ - Modelled with constant oceanic conductivity $\quad-\bullet-$ Modelled with depth-varying oceanic conductivity

- - Observed
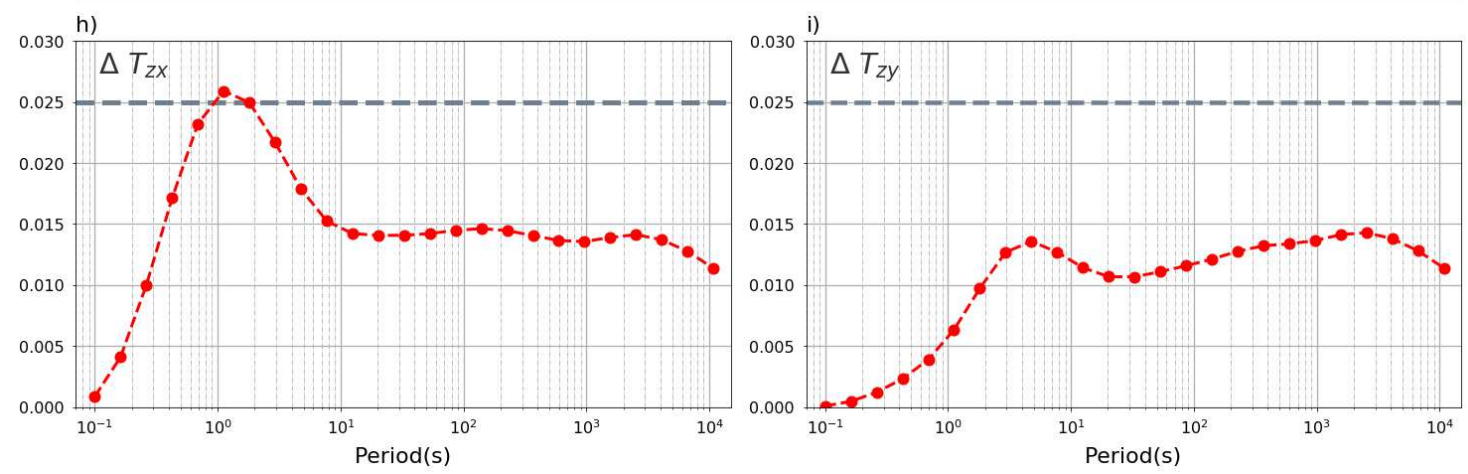

Figure 10. : Same as Figure 5, but for Easter Island observatory (IPM). 

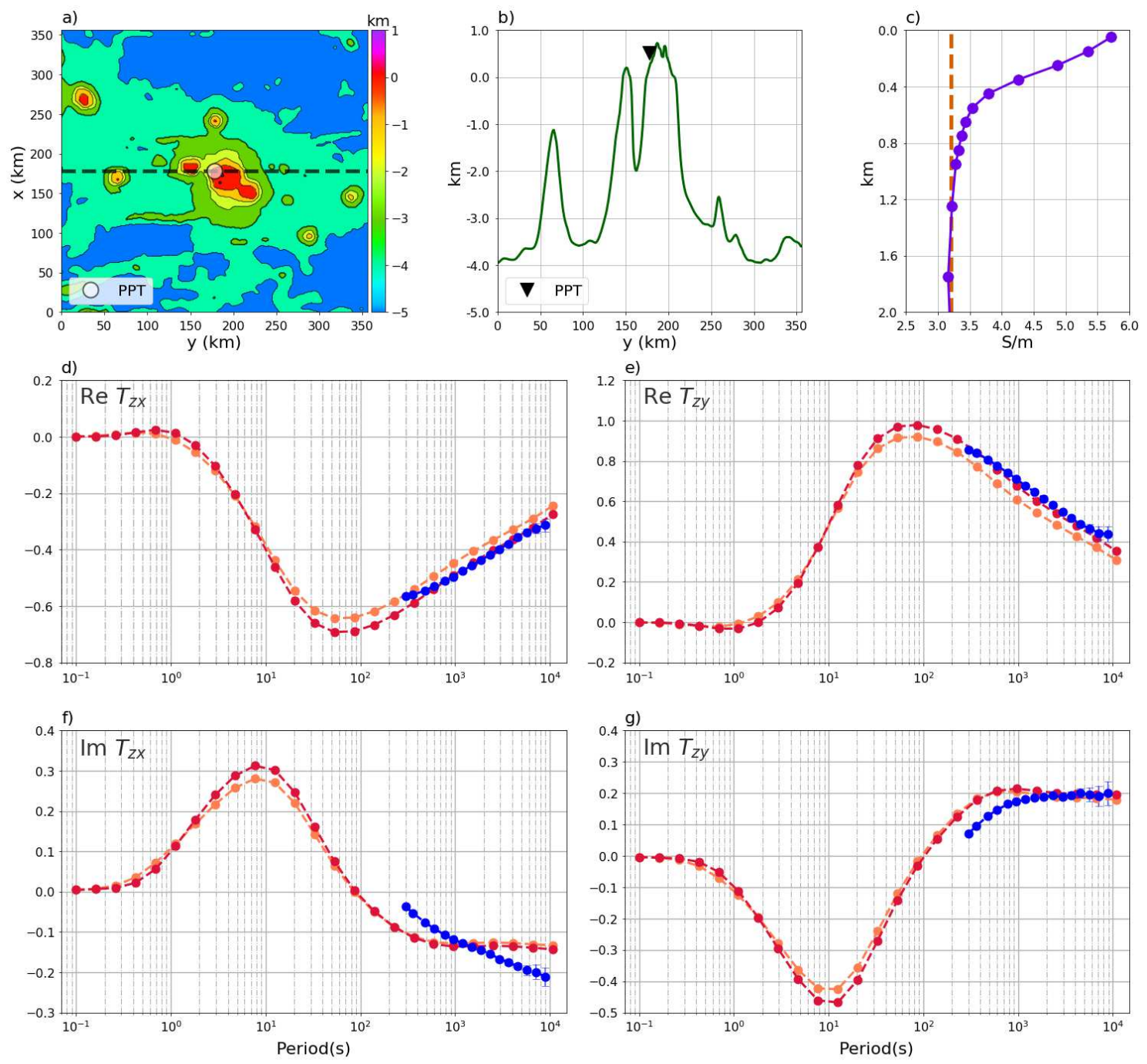

- - Modelled with constant oceanic conductivity $\quad-\bullet-$ Modelled with depth-varying oceanic conductivity - - Observed
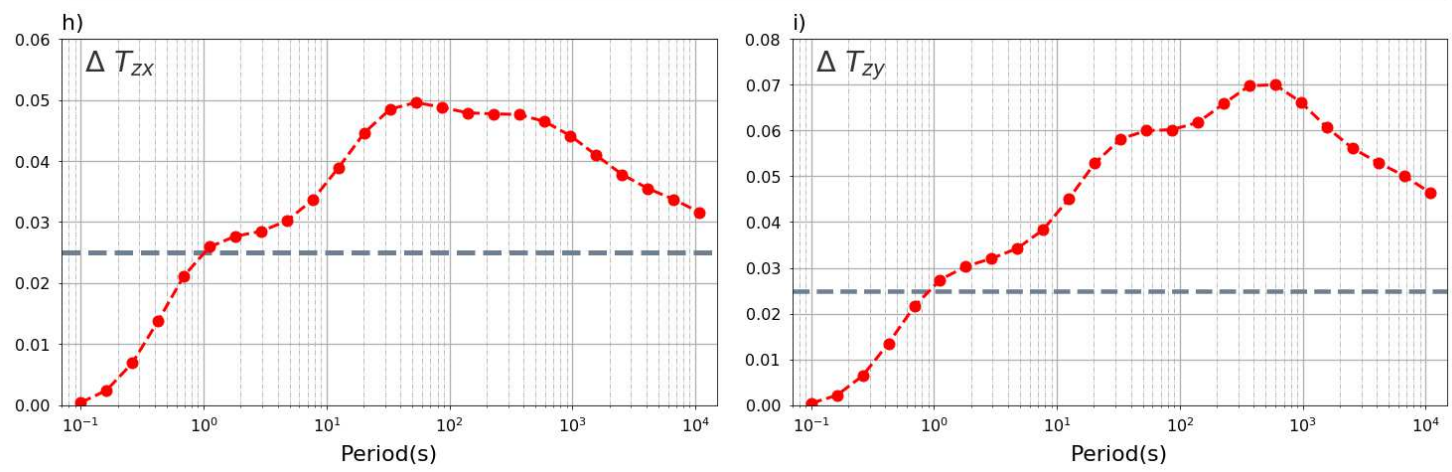

Figure 11. : Same as Figure 5, but for Pamatai observatory (PPT). 

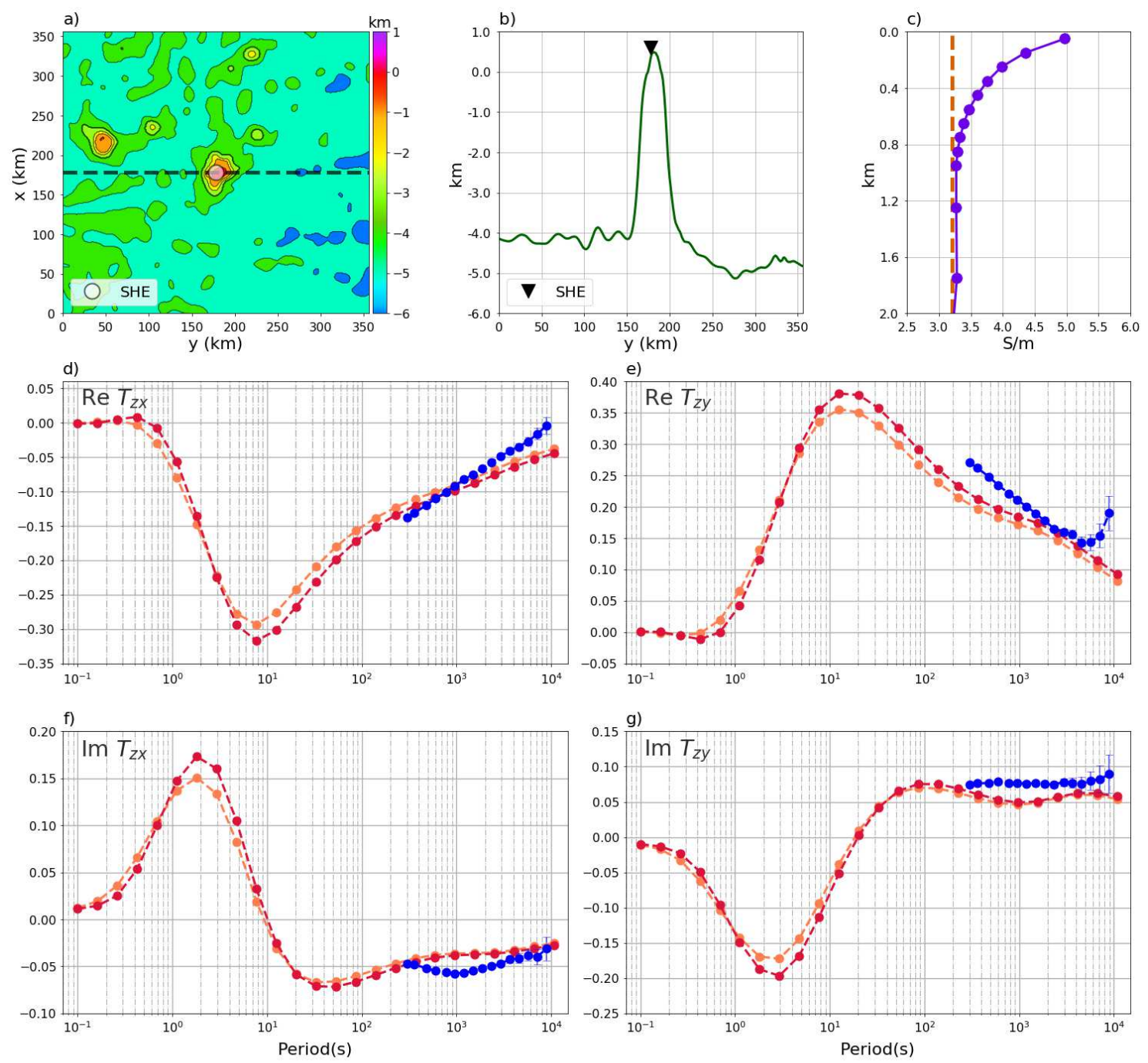

- - Modelled with constant oceanic conductivity - - - Modelled with depth-varying oceanic conductivity

- - Observed
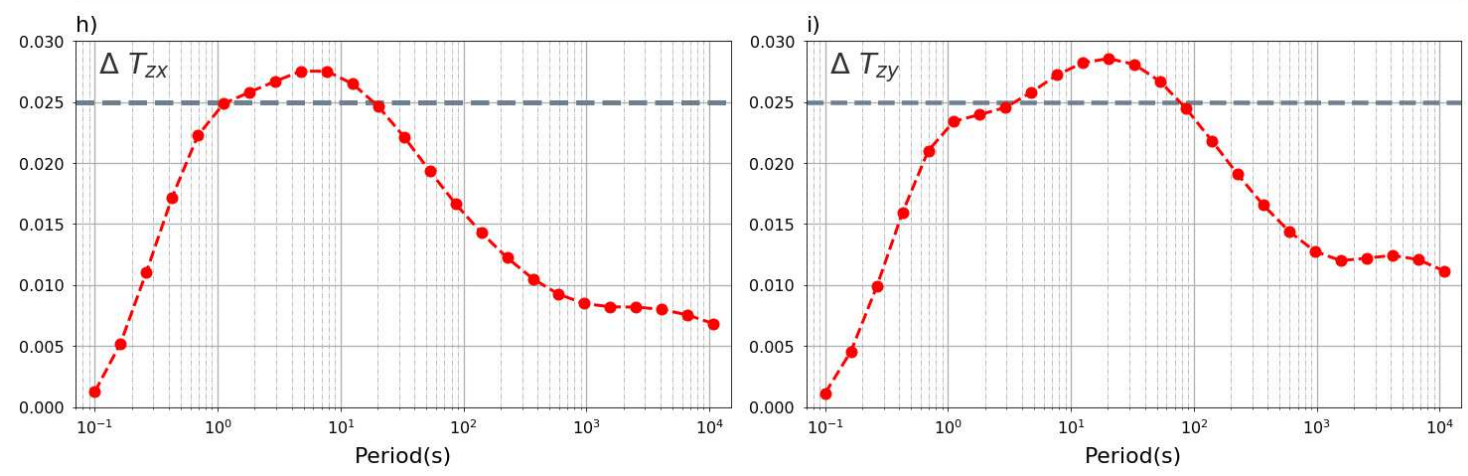

Figure 12. : Same as Figure 5, but for St. Helena observatory (SHE). 

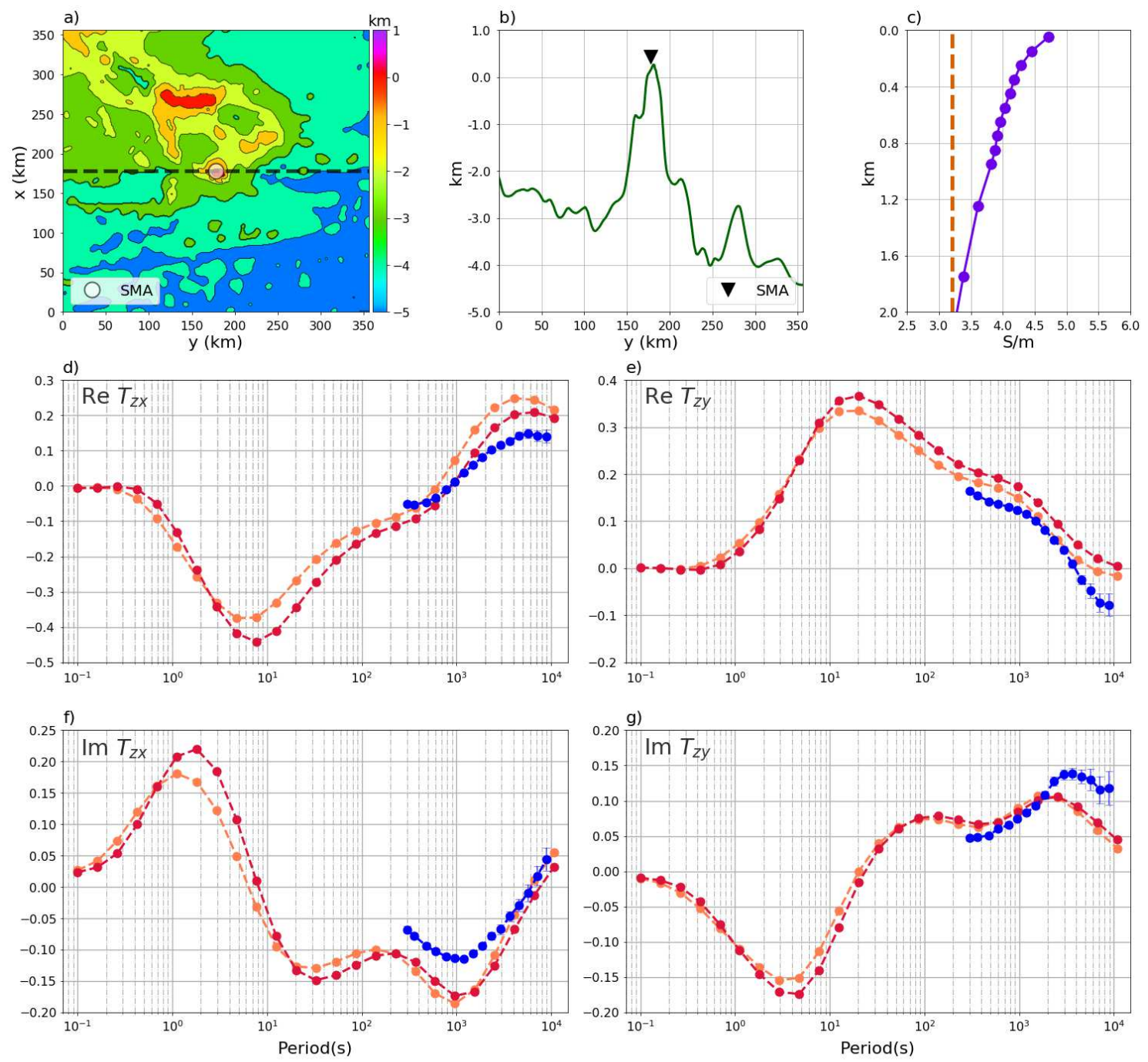

-๑ - Modelled with constant oceanic conductivity $\quad-\bullet-$ Modelled with depth-varying oceanic conductivity

- - Observed
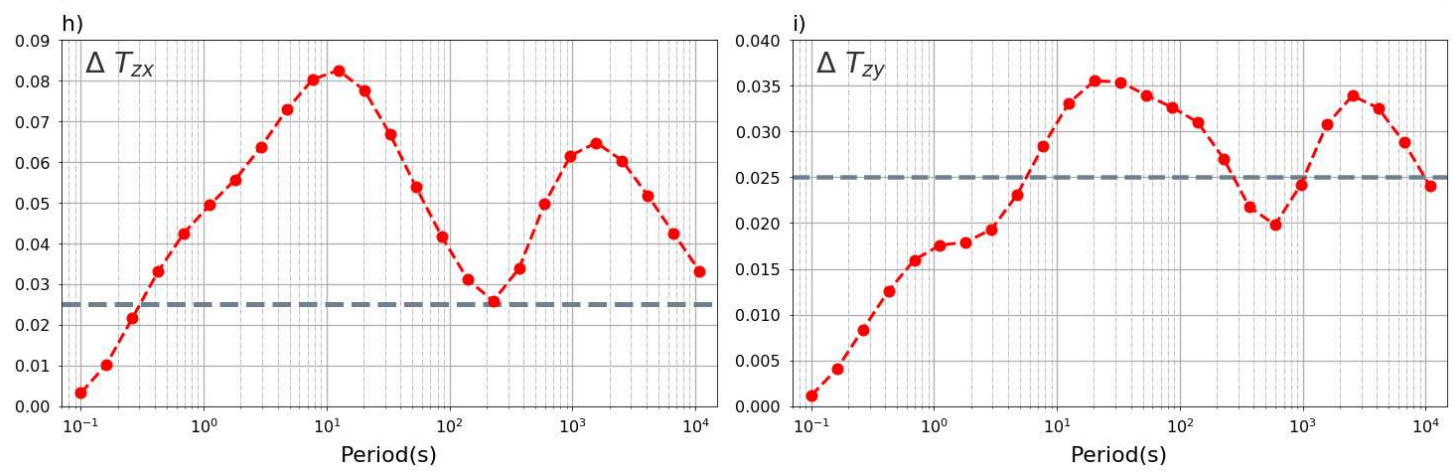

Figure 13. : Same as Figure 5, but for Santa-Maria/Azores observatory (SMA). 

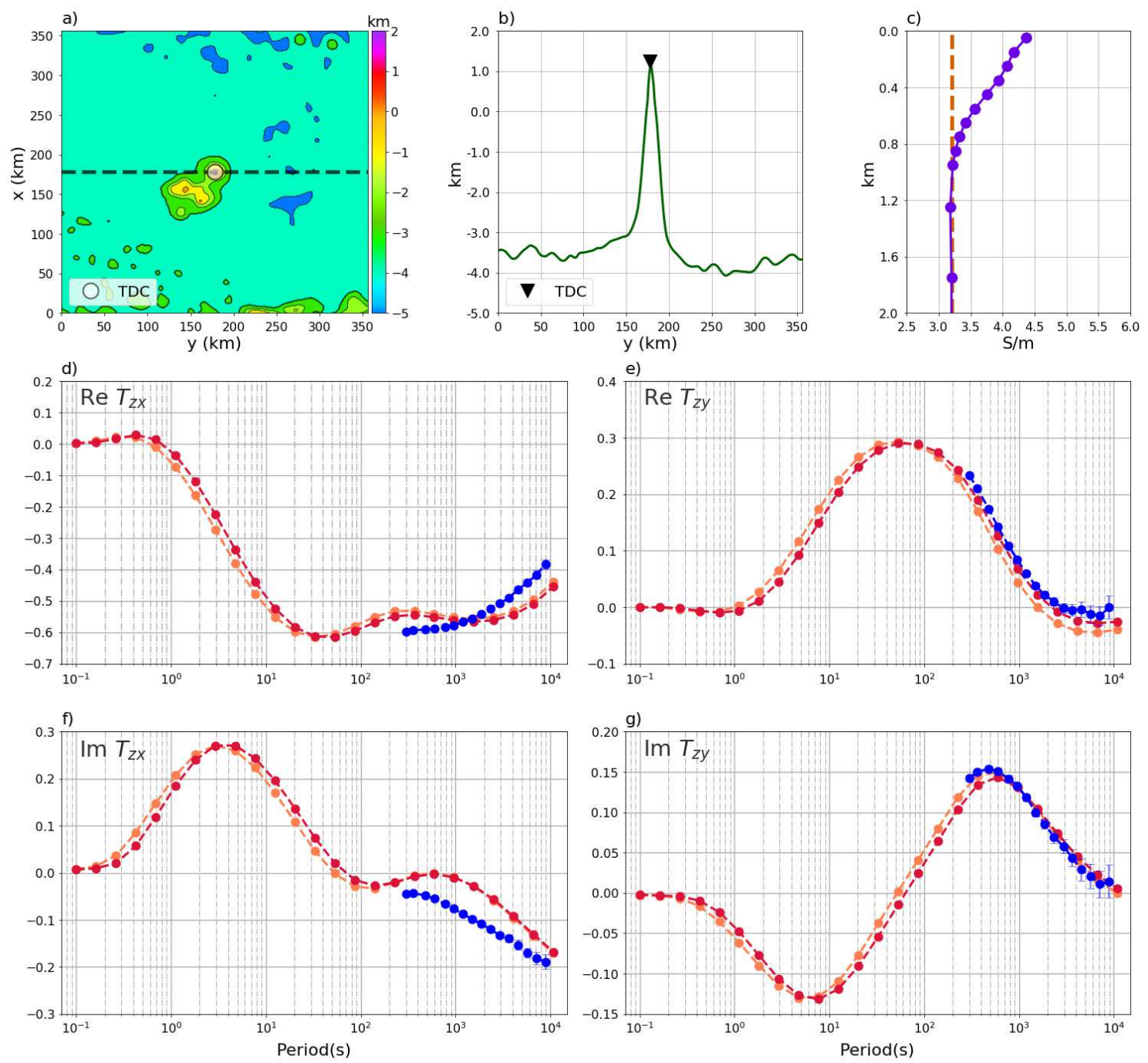

-๑ - Modelled with constant oceanic conductivity $\quad-\bullet-$ Modelled with depth-varying oceanic conductivity - - Observed
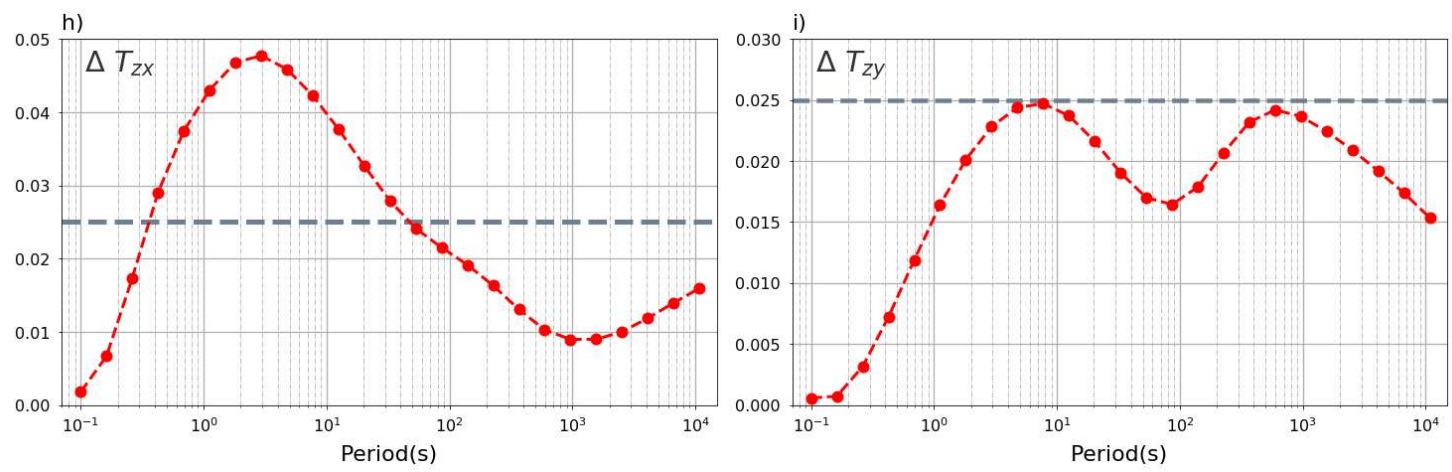

Figure 14. : Same as Figure 5, but for Tristan da Cunha observatory (TDC). 

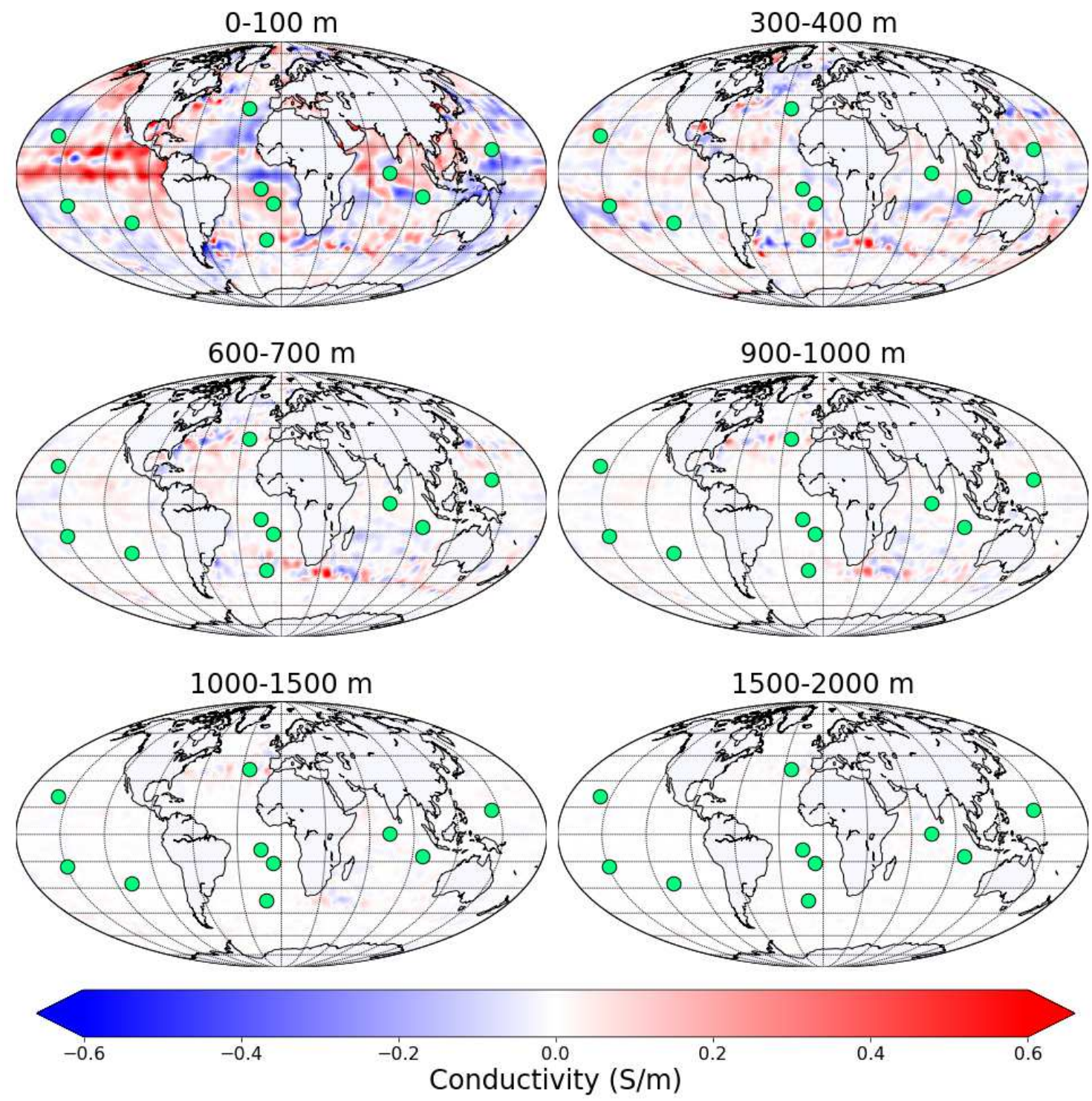

Figure 15. : Global maps of difference between 2015 December and June oceanic conductivity models at six depth intervals. Green circles denote locations of geomagnetic observatories used in this study. 

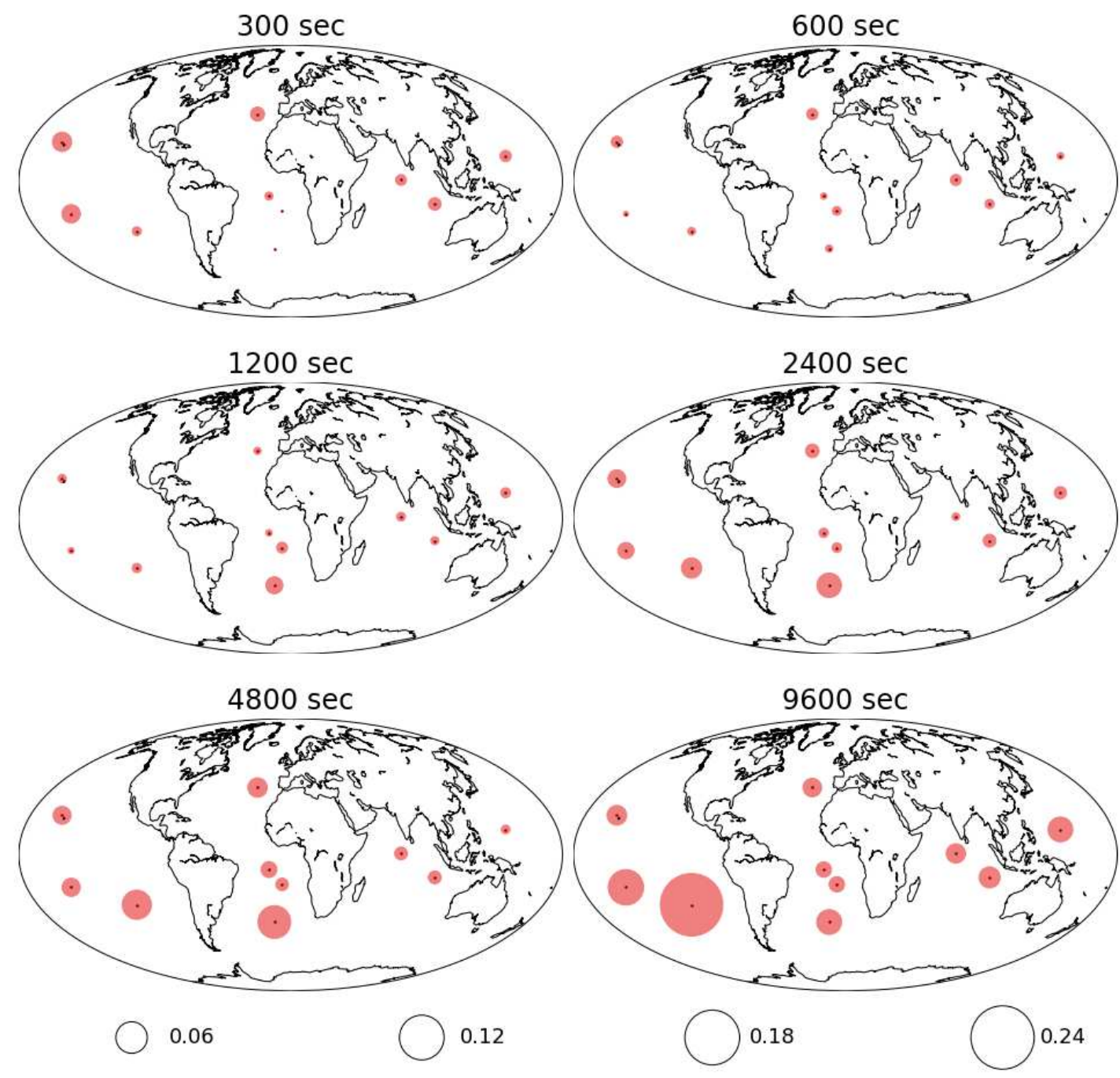

Figure 16. : Difference between "December" and "June" $T_{z x}$ at island observatories, shown as filled circles. "Experimental" and "modeled" differences are colored by light and dark red, respectively. The size of circles below the plots indicates four ranges of differences. 

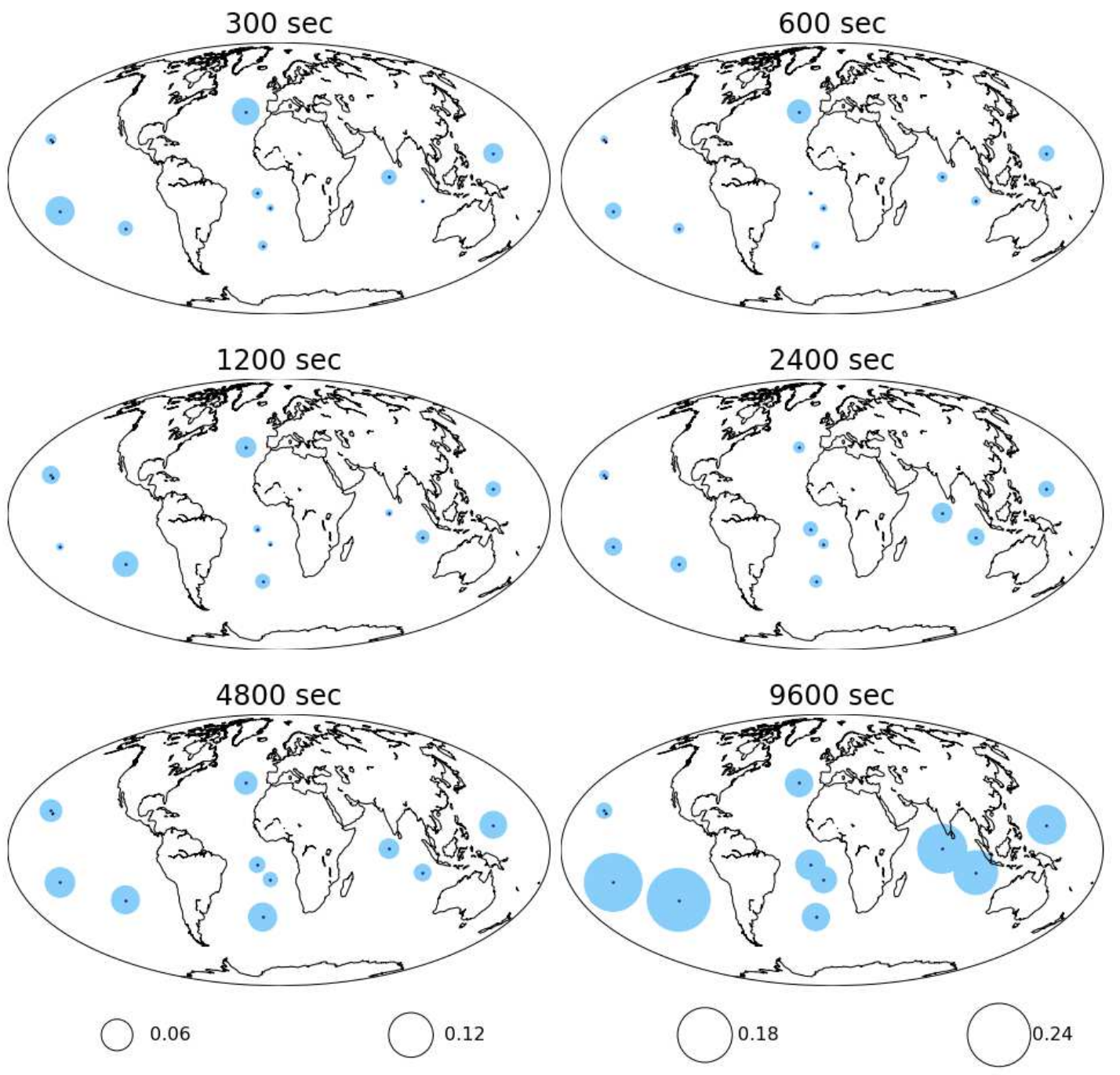

Figure 17. : Same as Figure 16, but for $T_{z y}$. 
Figures

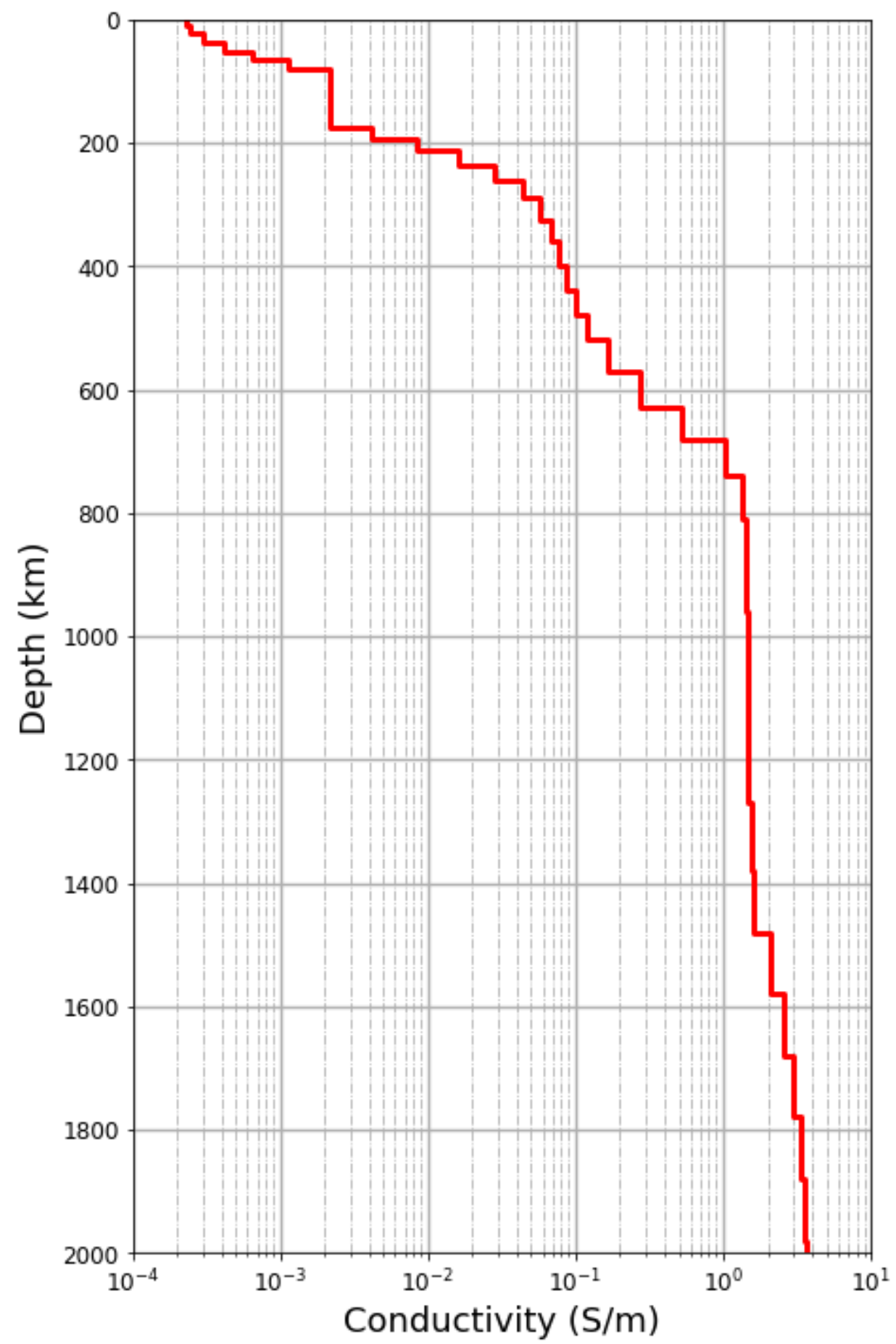

\section{Figure 1}

Location of geomagnetic observatories used in this study (green dots). Black lines depict $\pm 55 \square$ quasi dipole latitudes. Relevant information about these observatories is summarized in Table 1. 

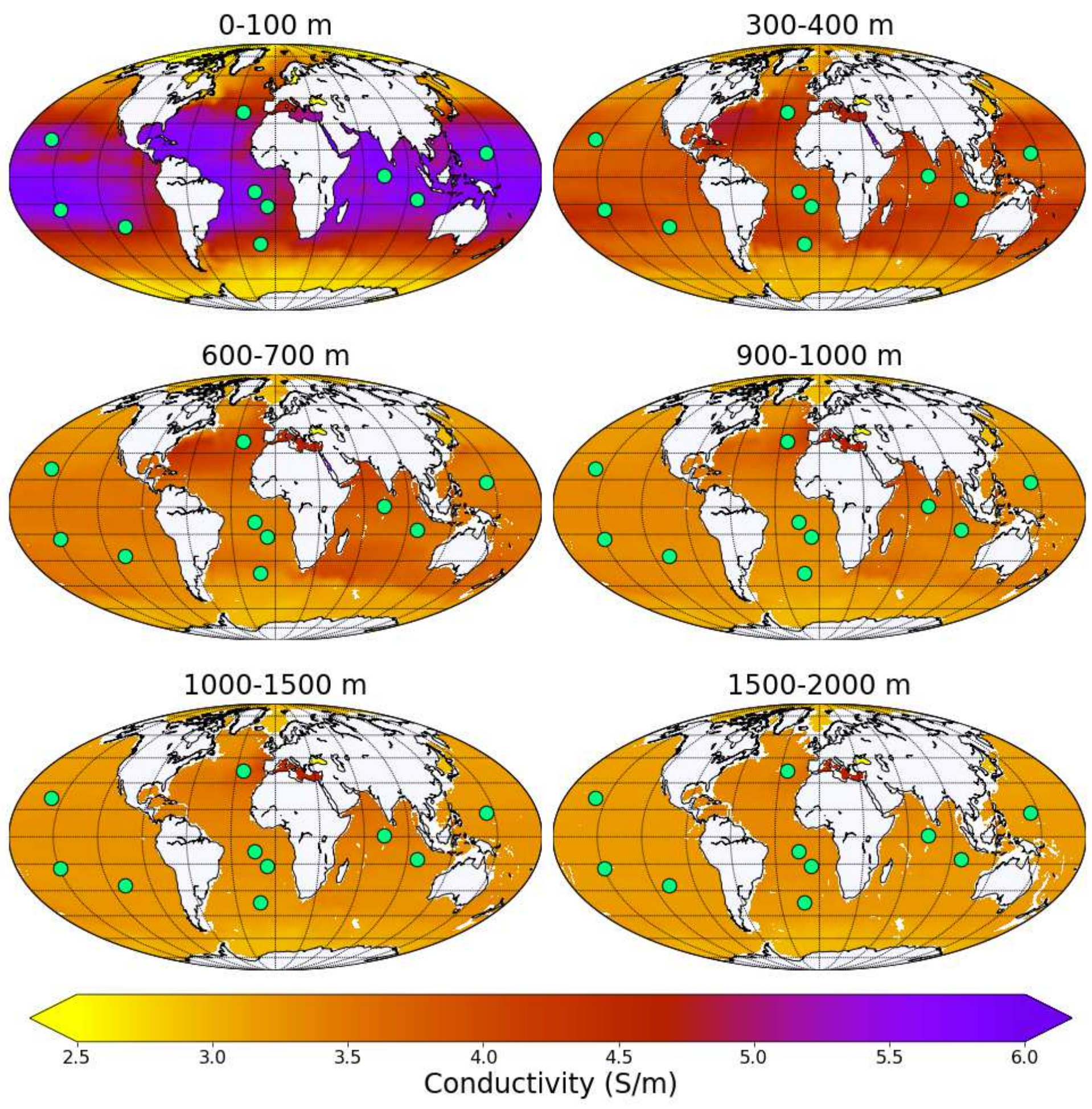

Figure 2

Global maps of oceanic electric conductivity for six selected depth intervals for December 2015 model. Green dots denote the locations of the geomagnetic observatories used in this study 


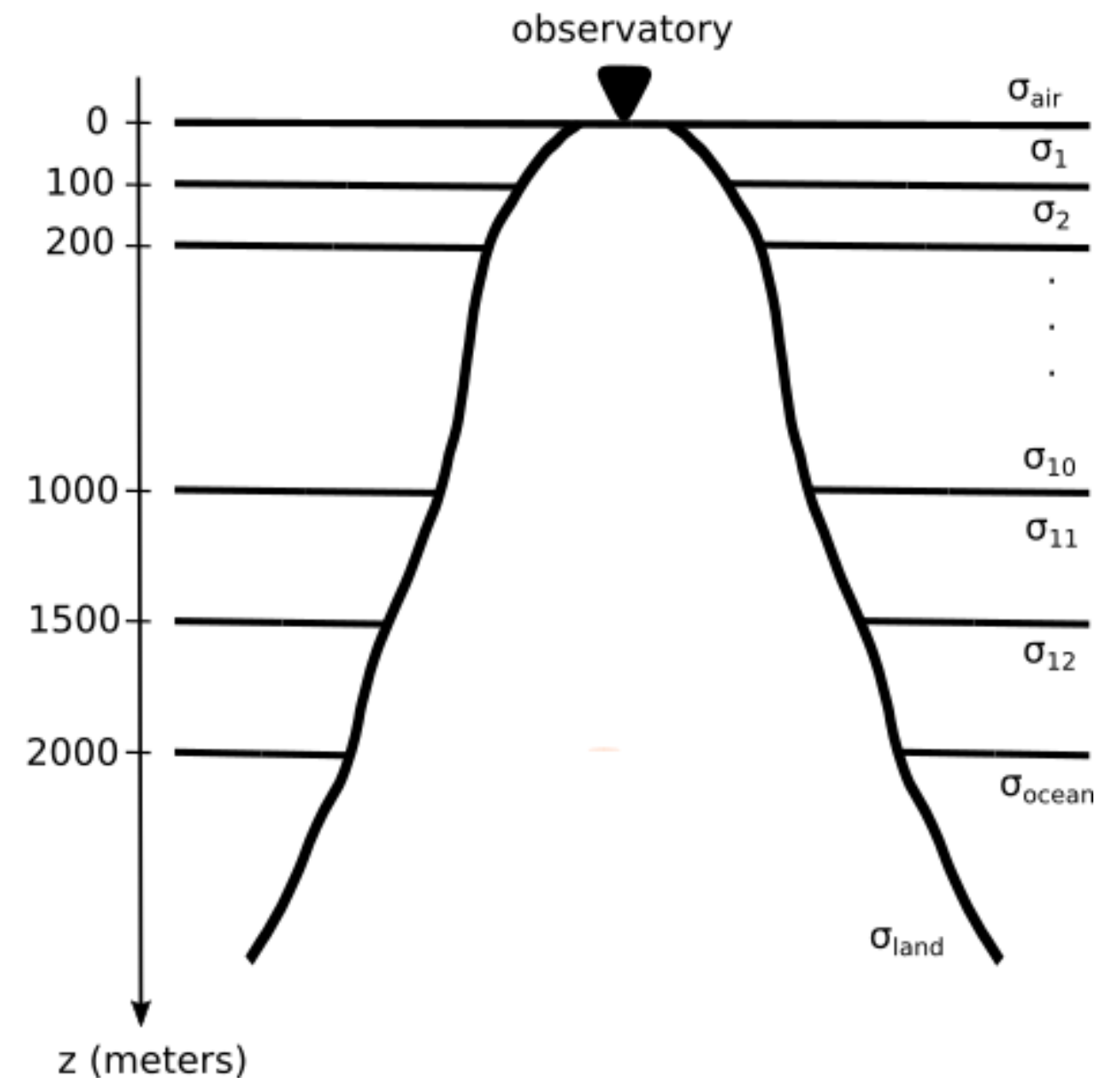

Figure 3

1-D global conductivity profile (from Grayver et al, 2017) used in this study for the crust and mantle beneath the 3-D (oceanic) modeling domain 


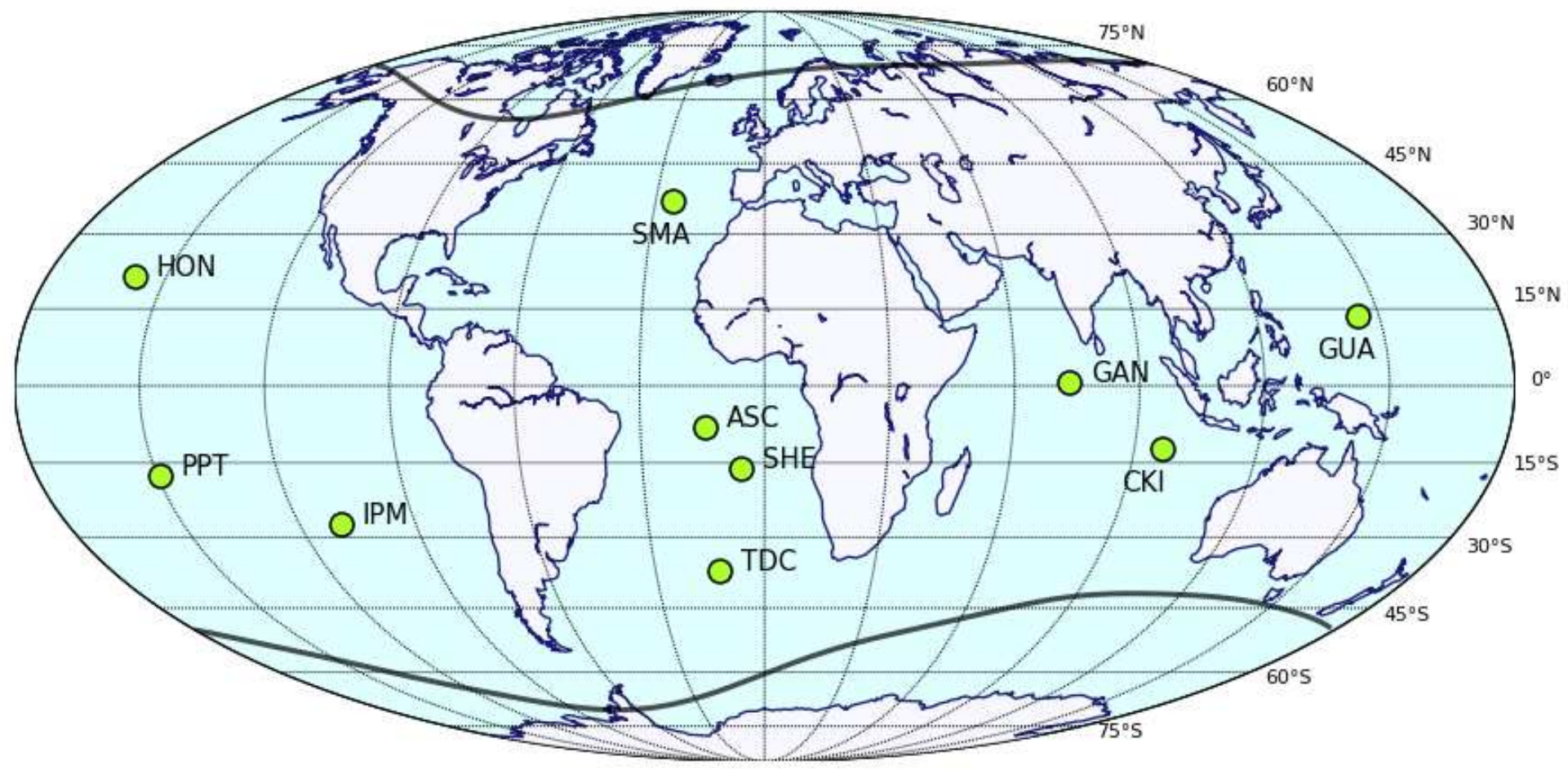

Figure 4

Vertical parametrization of the conductivity distribution in the 3-D modelling domain. $\sigma 1$ to $\sigma 12$ is the respective water layer's conductivity, set to the layers's average conductivity calculated from the global ocean conductivity model. бocean is the deep (below $2000 \mathrm{~m}$ ) ocean conductivity set to $3.2 \mathrm{~S} / \mathrm{m}$. oland is the landmass conductivity set to $0.01 \mathrm{~S} / \mathrm{m}$. 

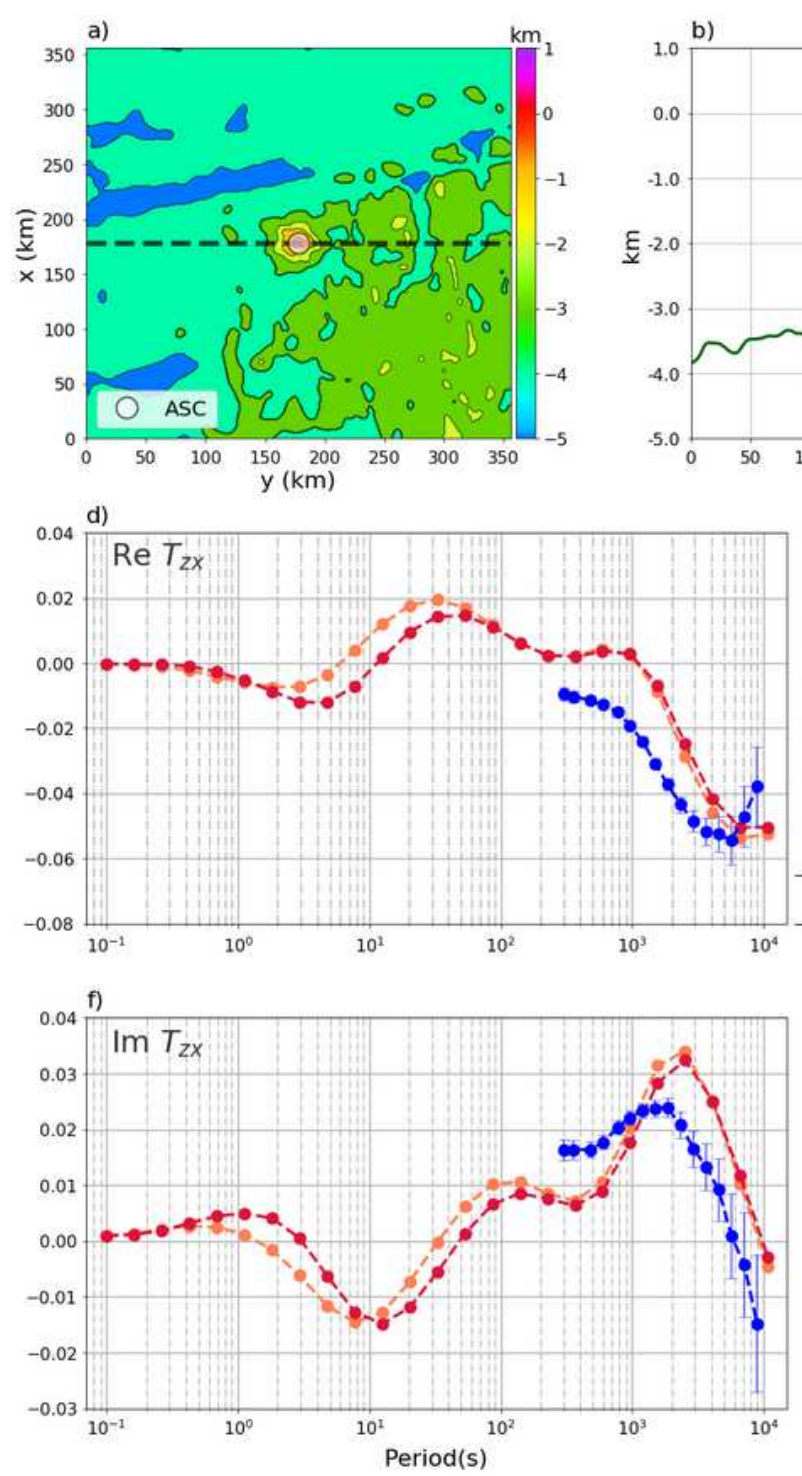

- - Modelled with constant oceanic conductivity

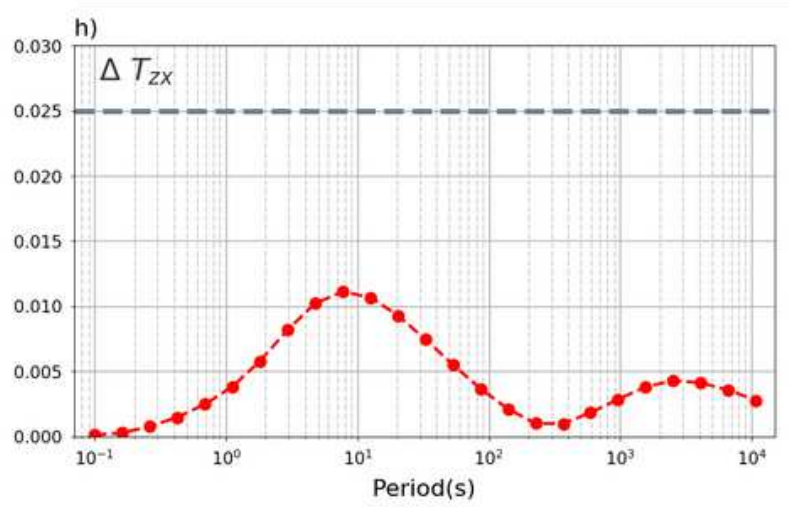

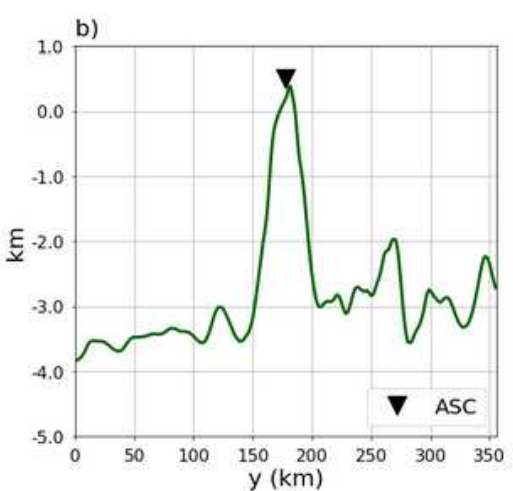
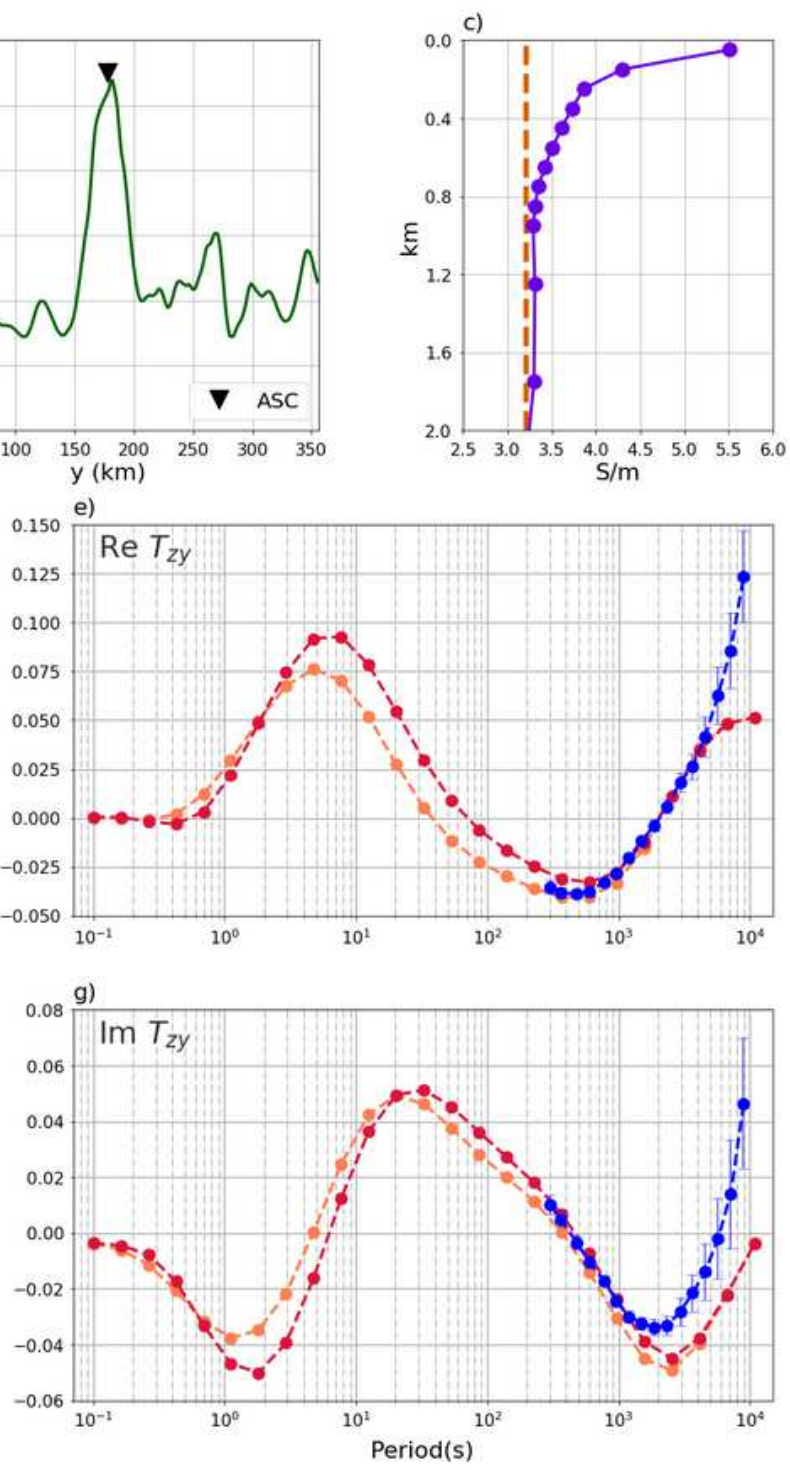

- Modelled with depth-varying oceanic conductivity

- - Observed

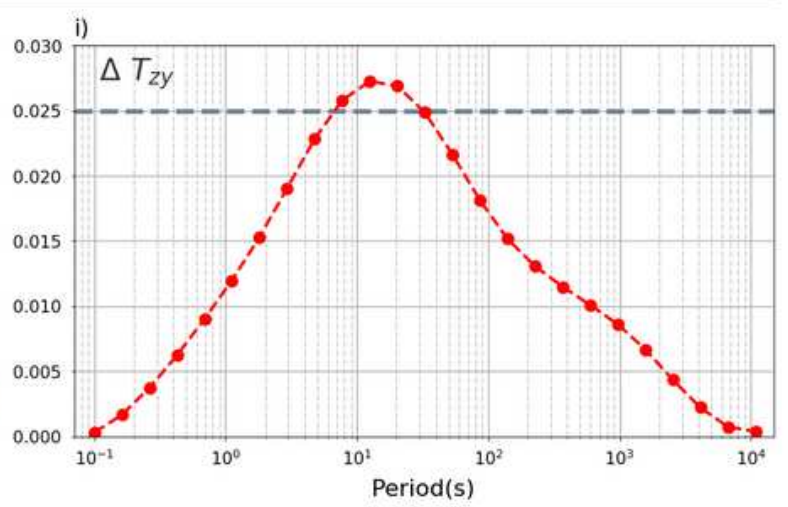

\section{Figure 5}

Results for Ascension Island observatory (ASC). (a) Map of bathymetry; dashed line indicates location of profile shown in panel (b). (b) West-East oriented bathymetry profile. (c) Regional depth-varying (purple) oceanic conductivity and constant reference oceanic conductivity $(3.2 \mathrm{~S} / \mathrm{m}$, red dashed line). (d) to (g) Real and imaginary parts for $\mathrm{x}$ and $\mathrm{y}$ components of tippers computed in the model with depth-varying (orange dots and dashed line) and depth-constant (red dots and dashed line) oceanic conductivity. (h) 
Difference for the computed tipper $x$ component between depth-varying and depth-constant ocean conductivity, see text for details. Dashed grey line indicates the threshold of 0.025. (i) Same as (h), but for the y component.
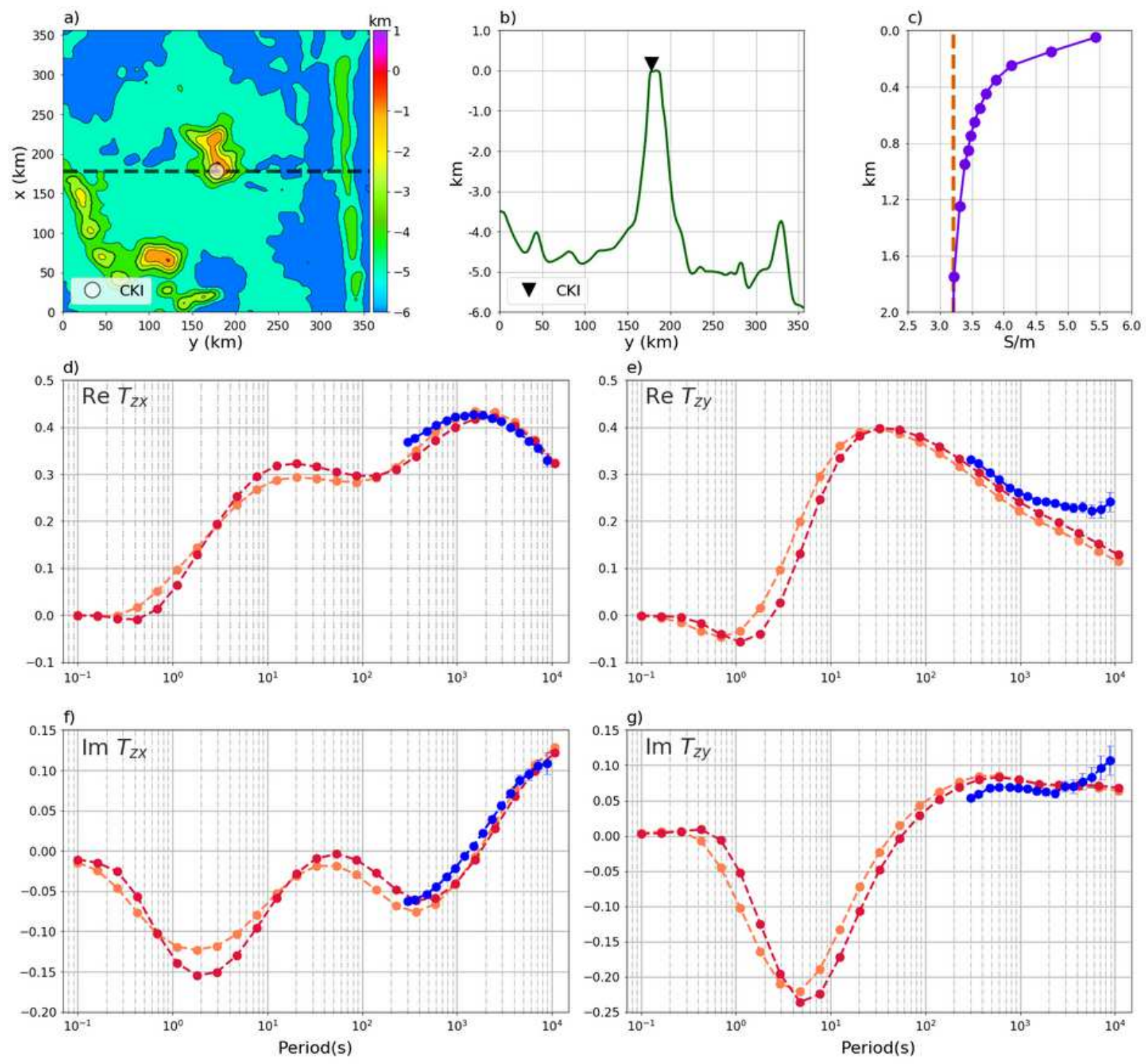

- - Modelled with constant oceanic conductivity

- - Modelled with depth-varying oceanic conductivity

-๑- Observed
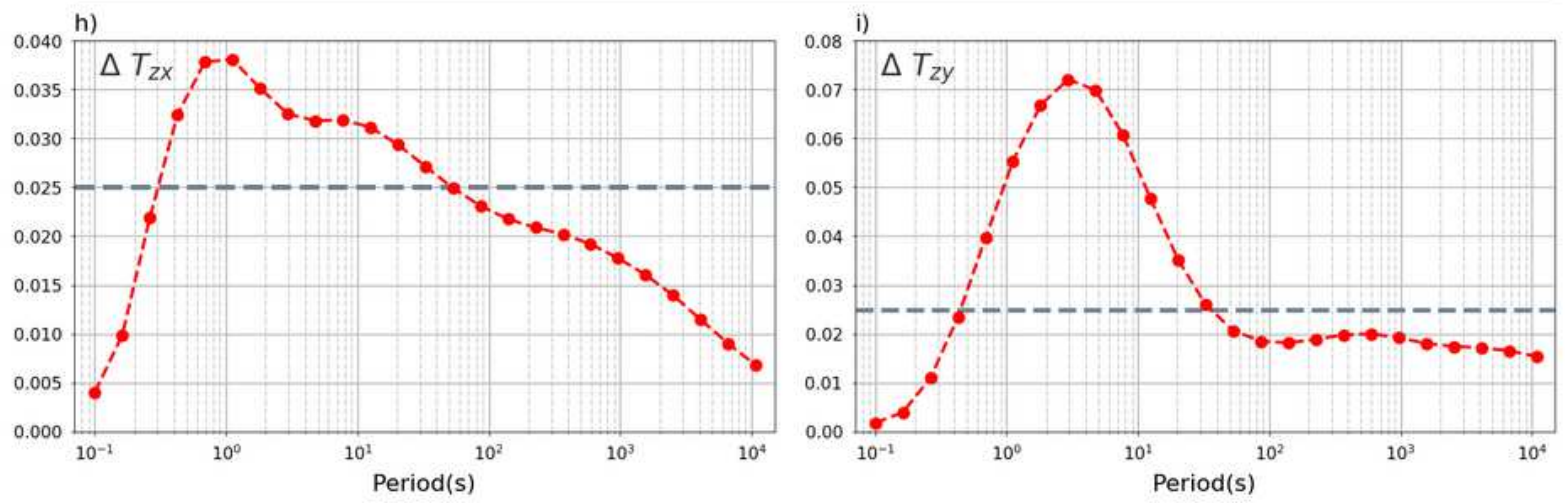

Figure 6

Same as Figure 5, but for Cocos-Keeling Islands observatory (CKI). 

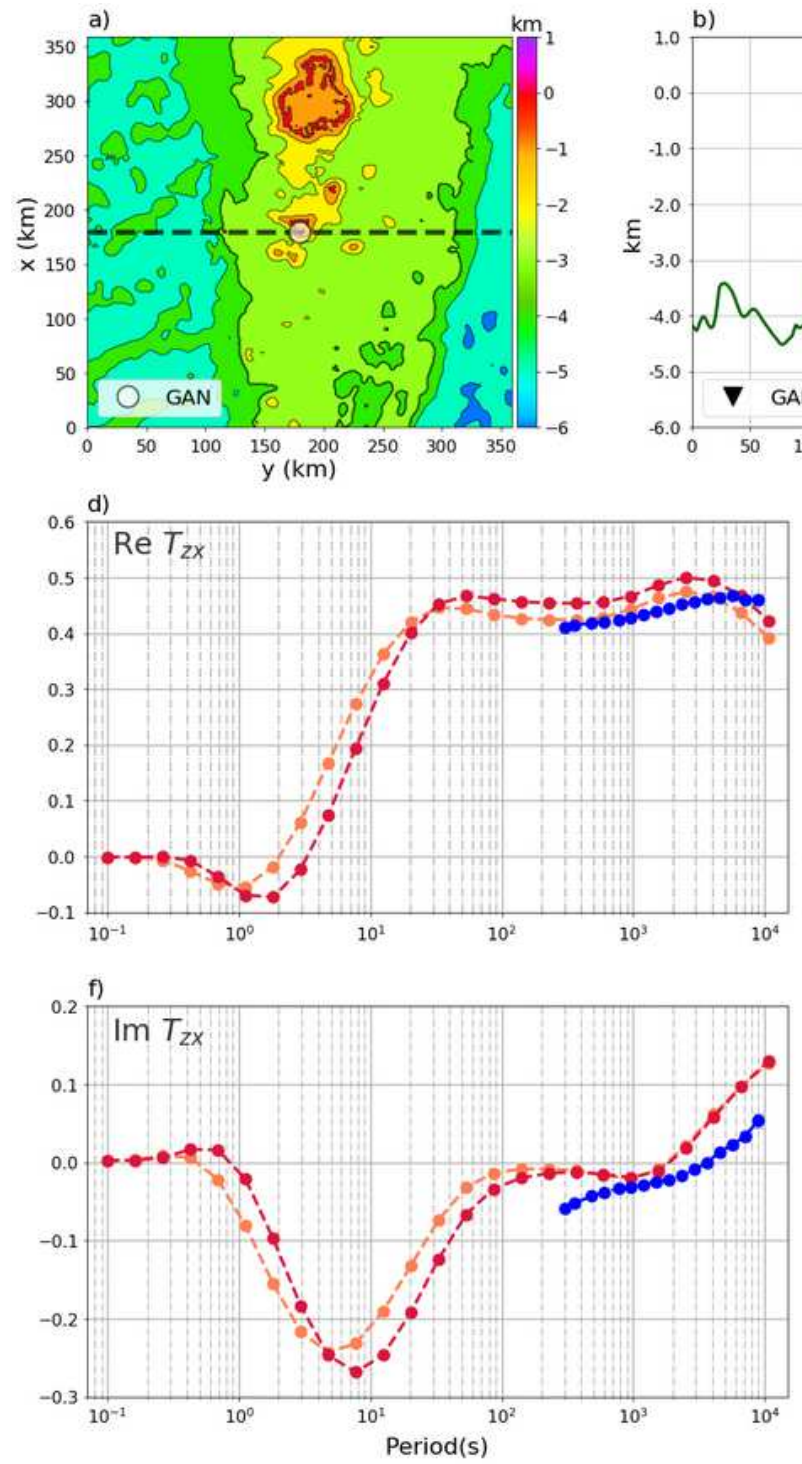

- - Modelled with constant oceanic conductivity

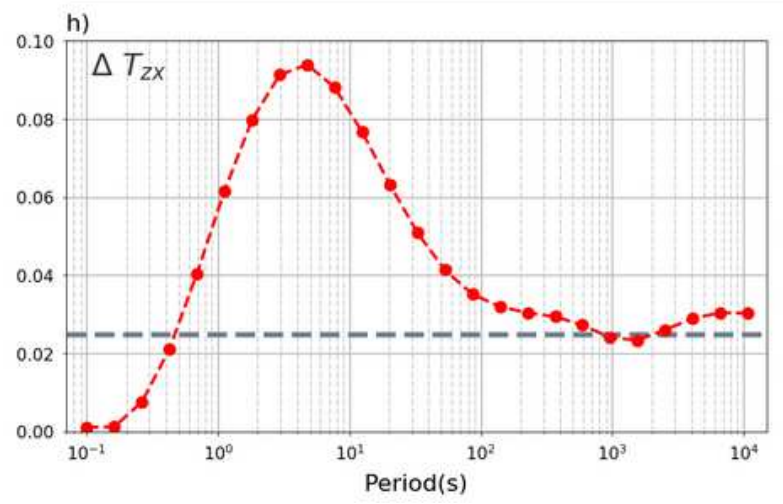

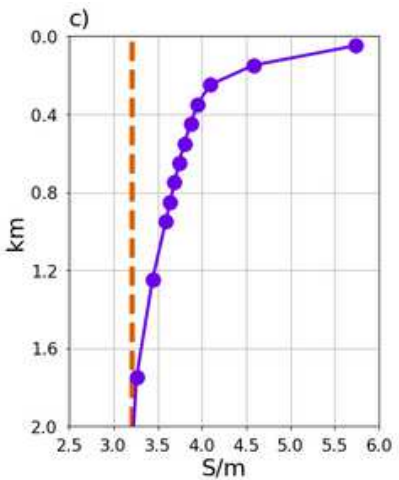
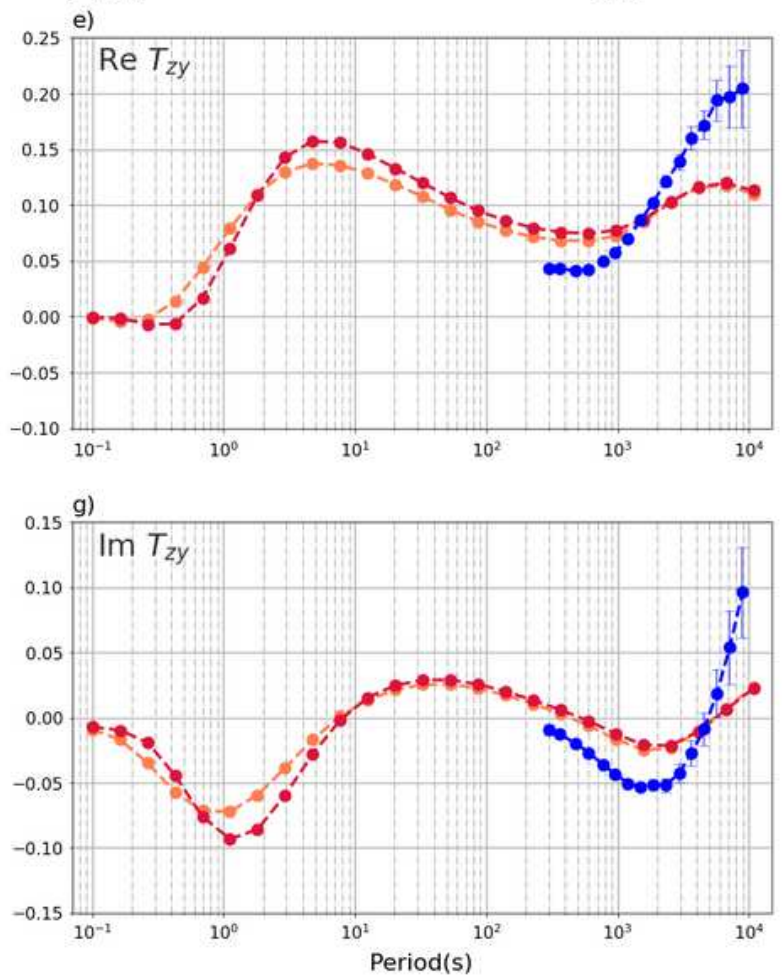

Period(s)

odelled with depth-varying oceanic conductivity

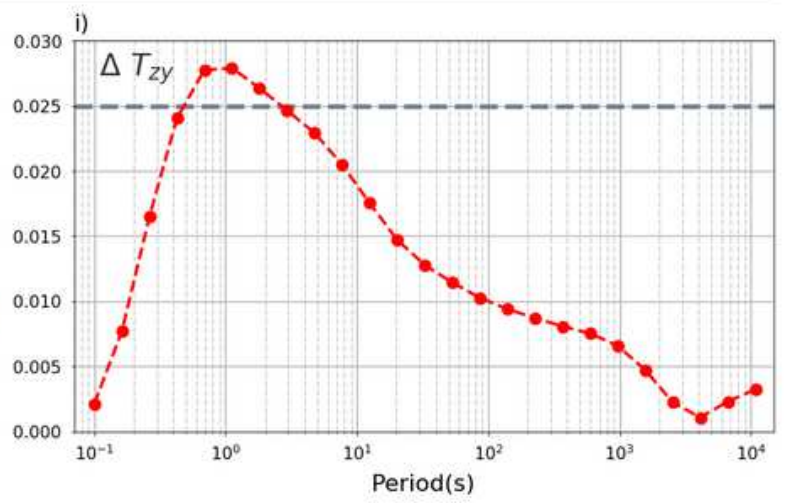

\section{Figure 7}

Same as Figure 5, but for Gan observatory (GAN). 

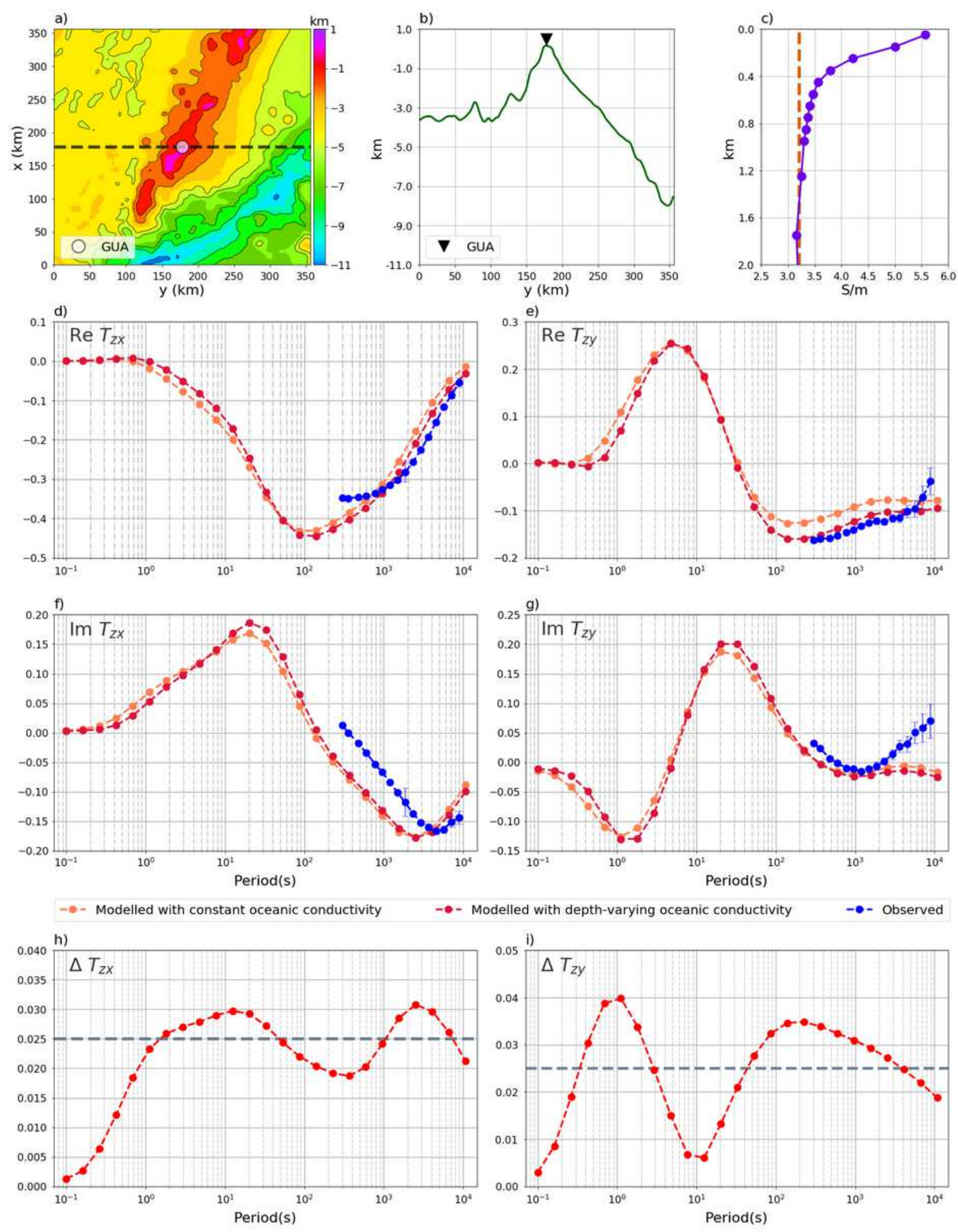

Figure 8

Same as Figure 5, but for Guam observatory (GUA). 

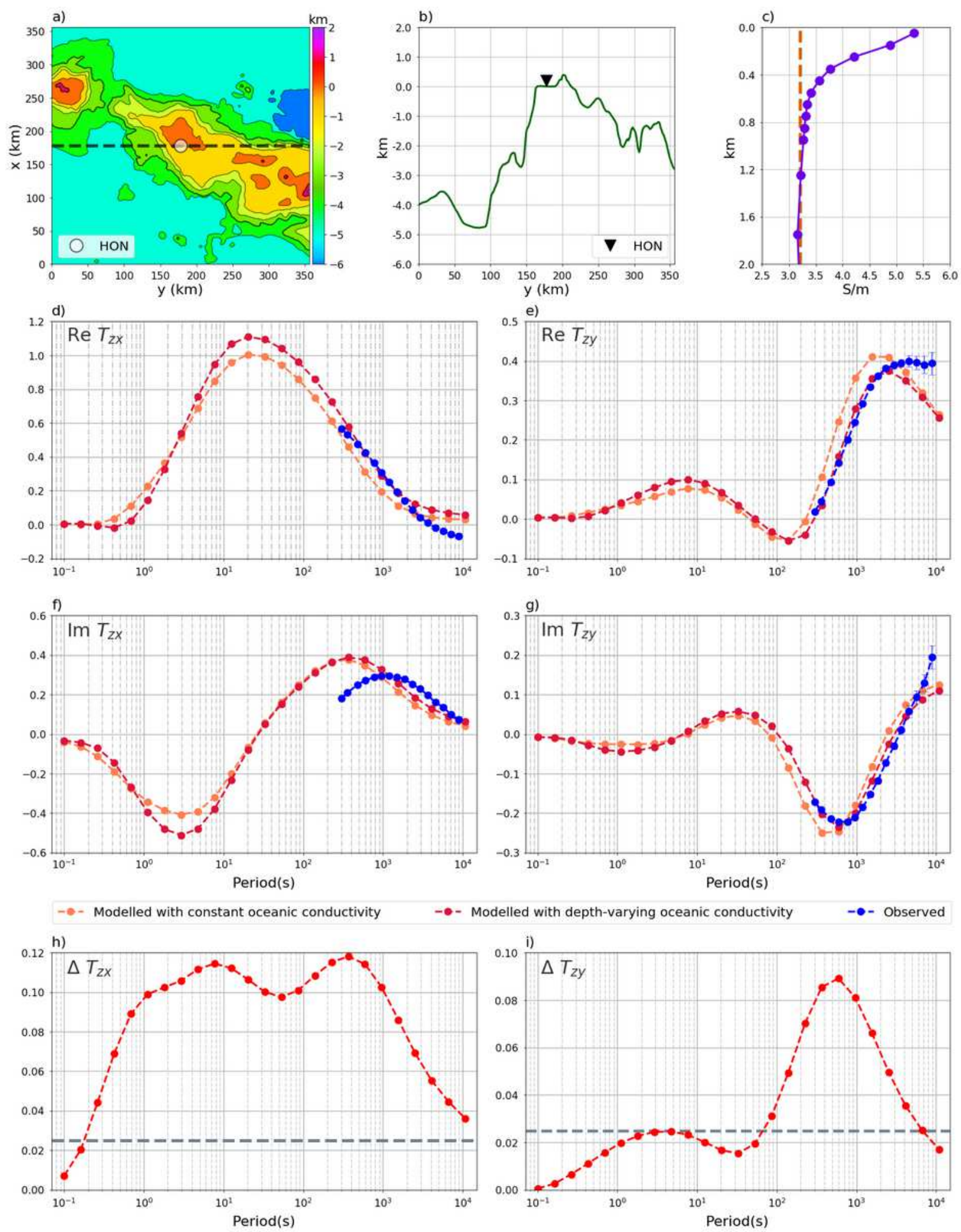

Figure 9

Same as Figure 5, but for Honolulu observatory (HON). 

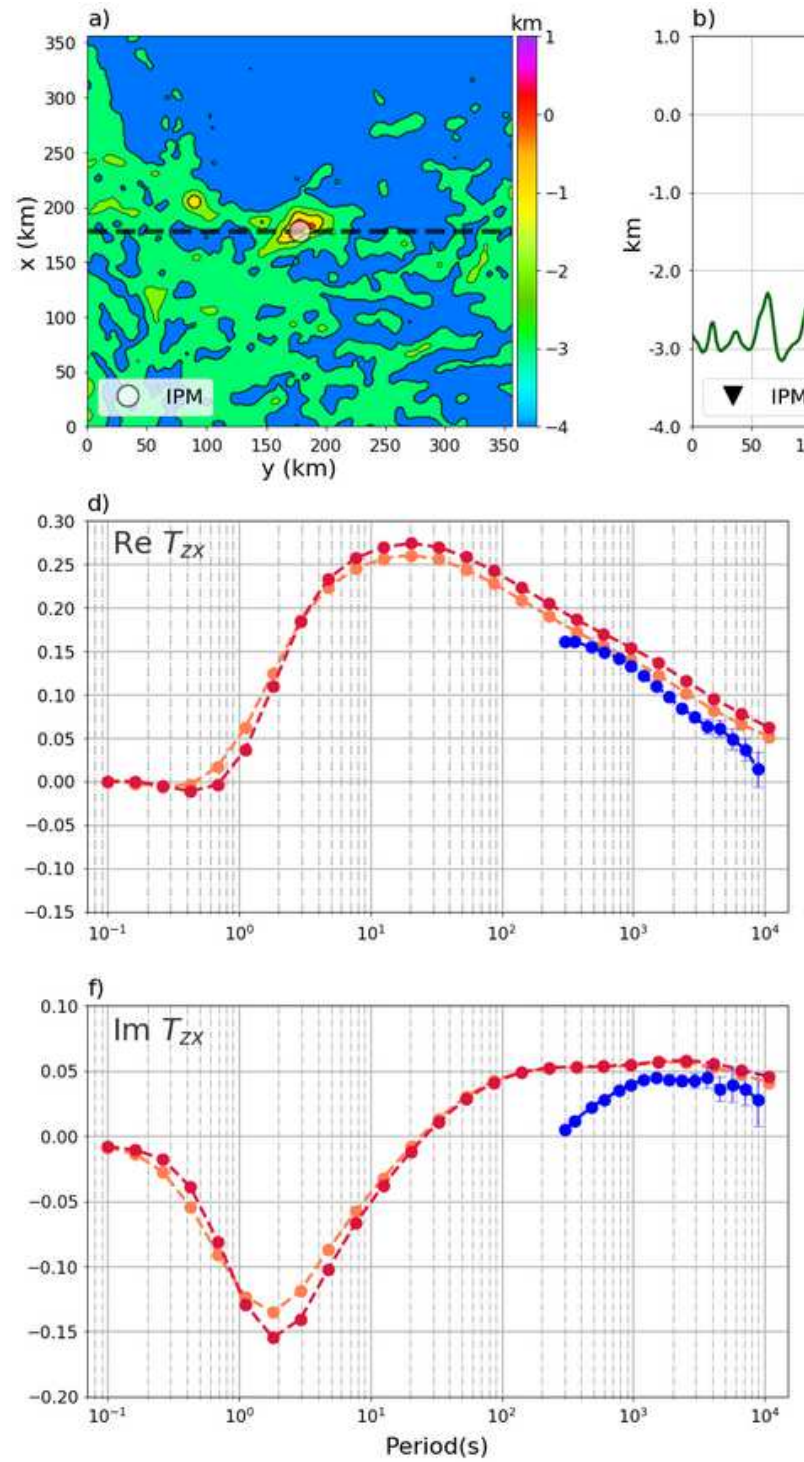

- - Modelled with constant oceanic conductivity

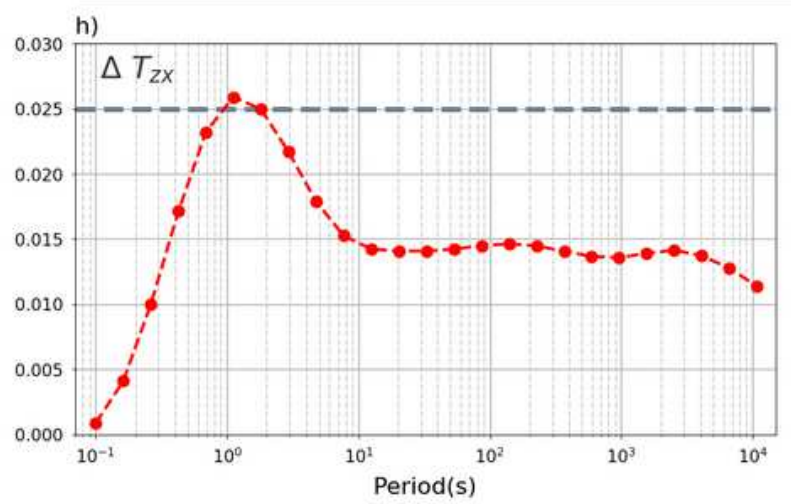

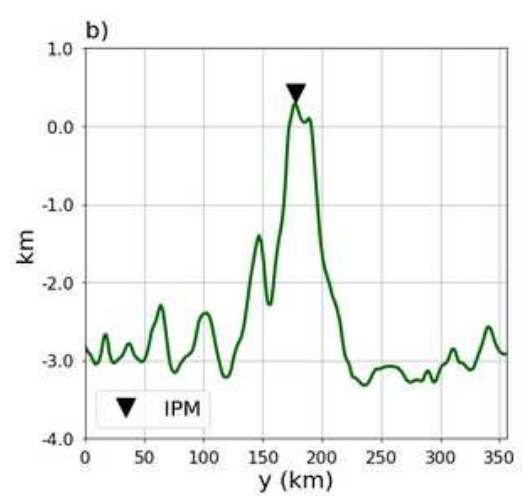
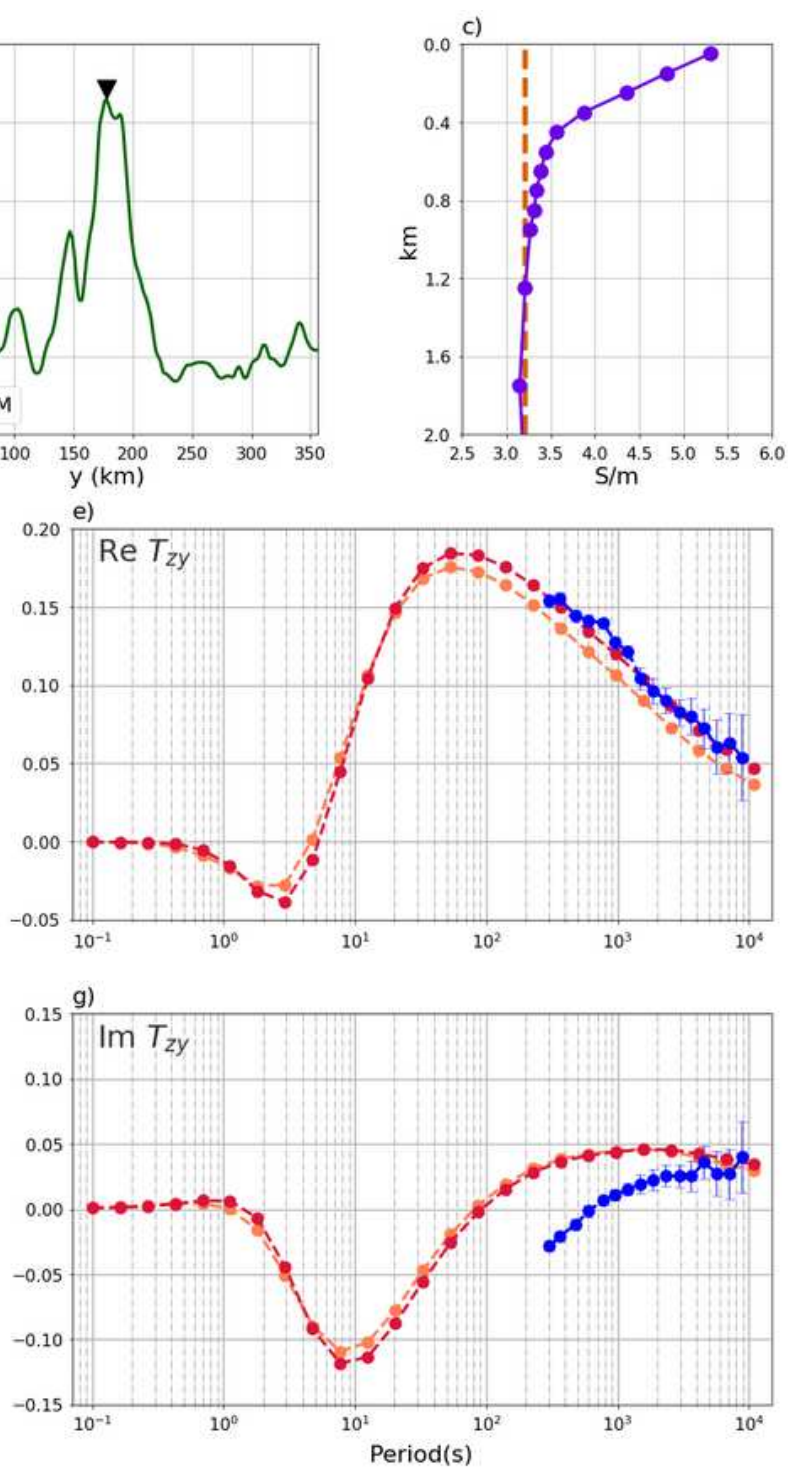

Period(s) 

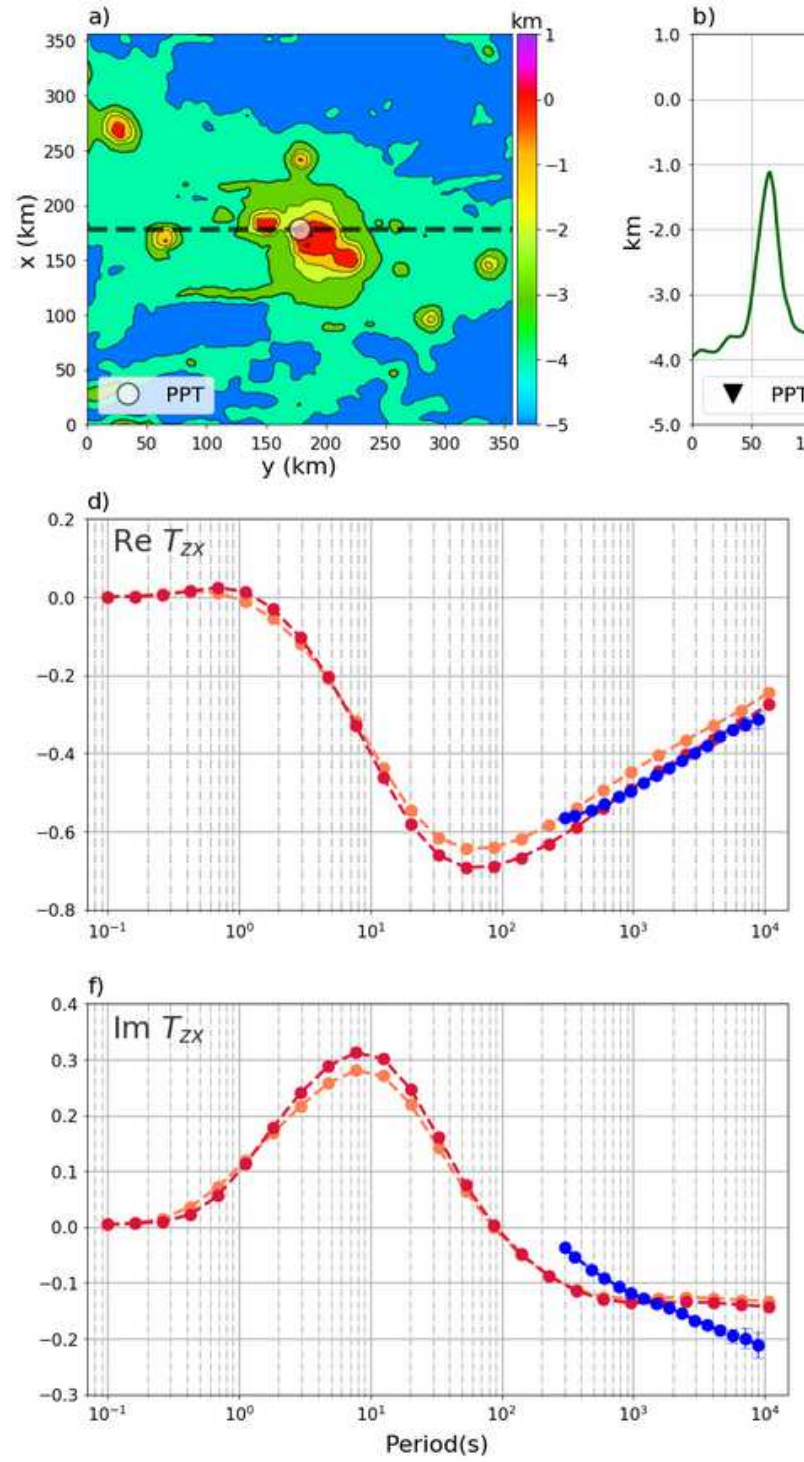

- - Modelled with constant oceanic conductivity

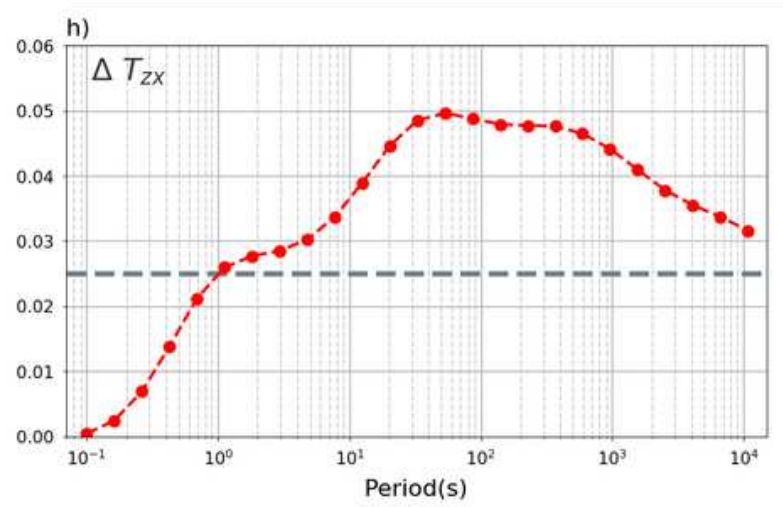

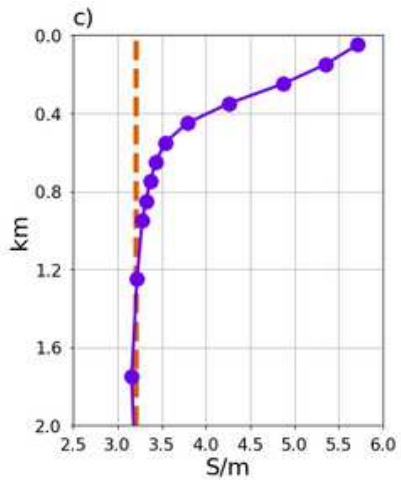
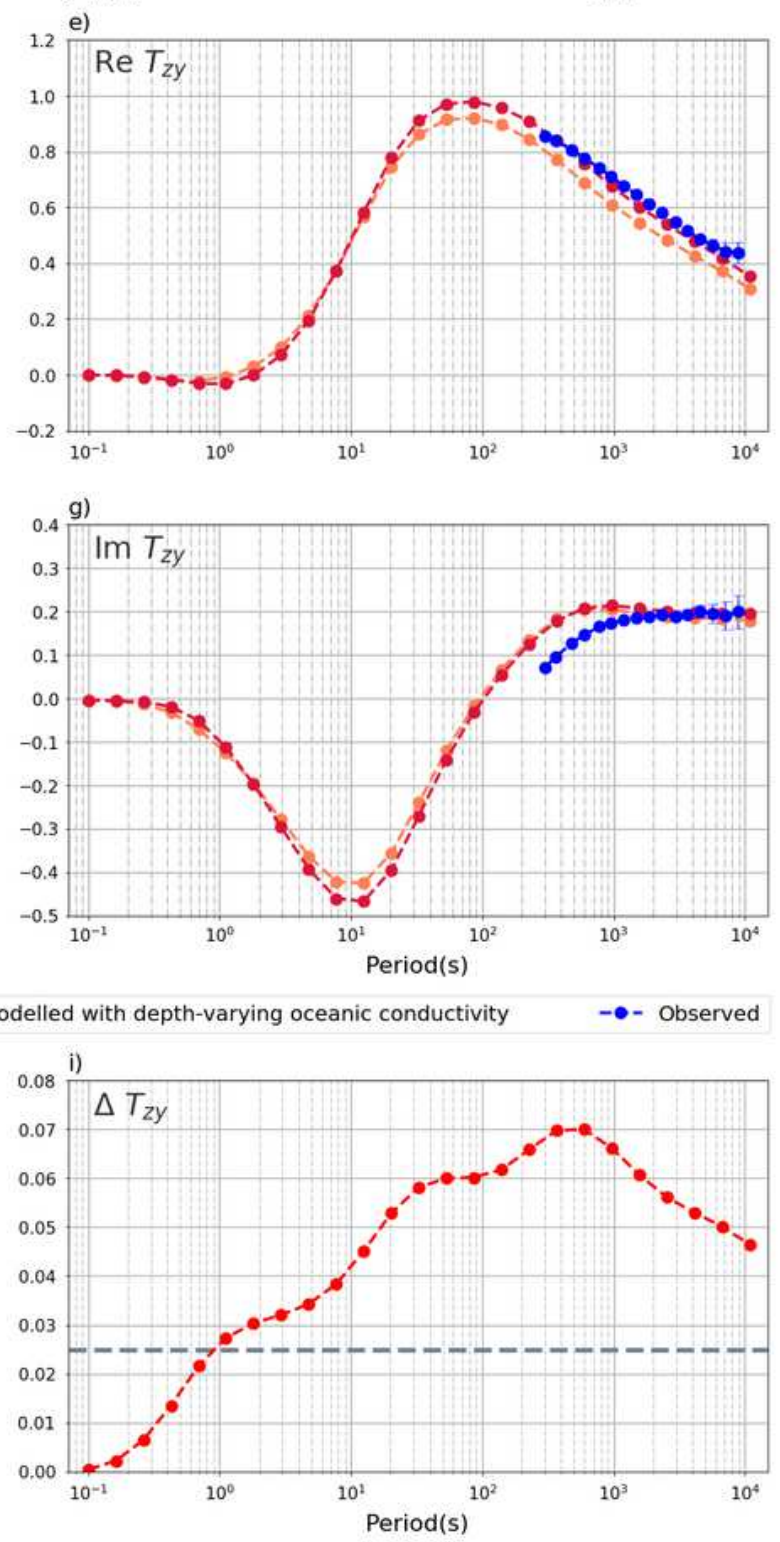

\section{Figure 11}

Same as Figure 5, but for Pamatai observatory (PPT). 

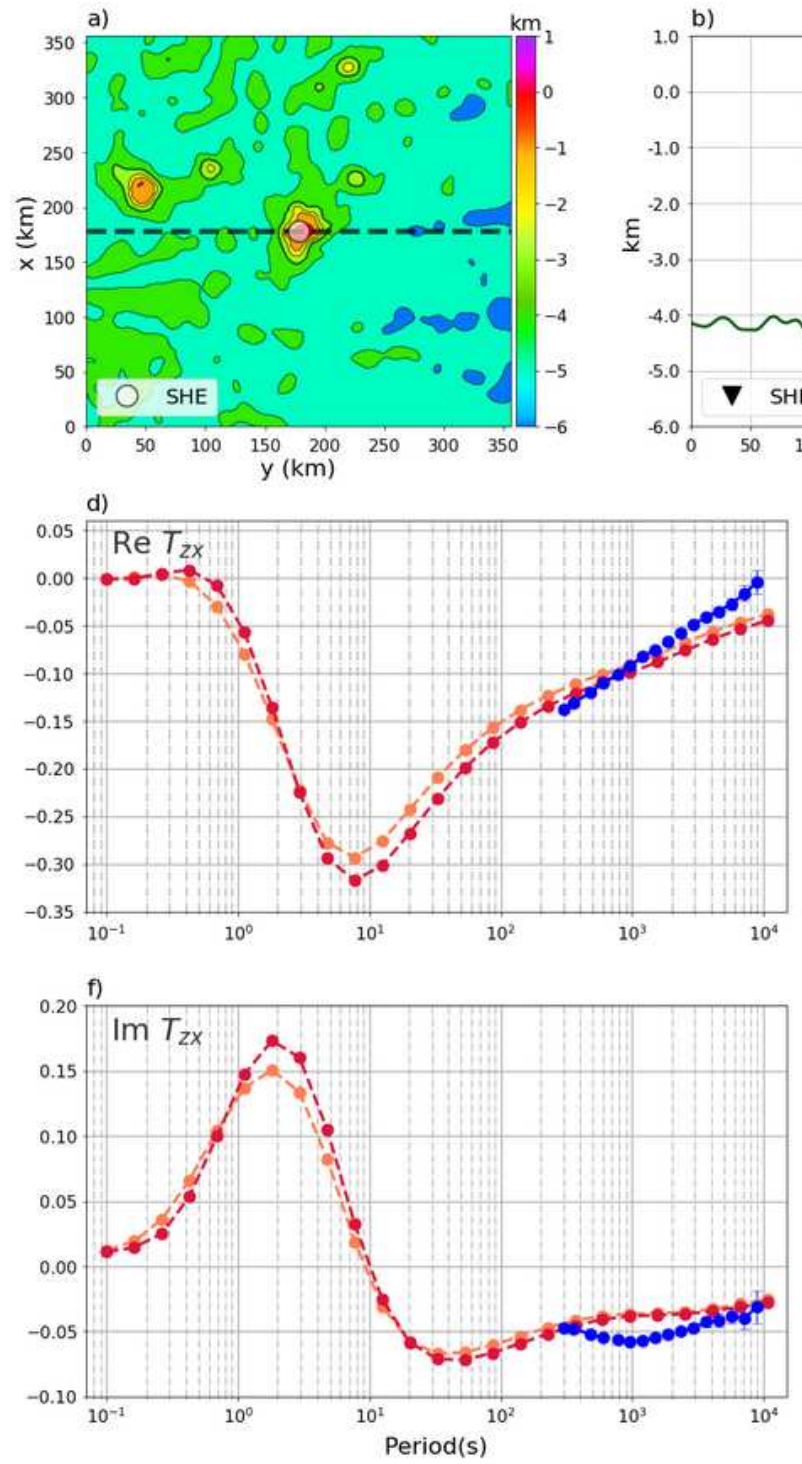

Period(s)
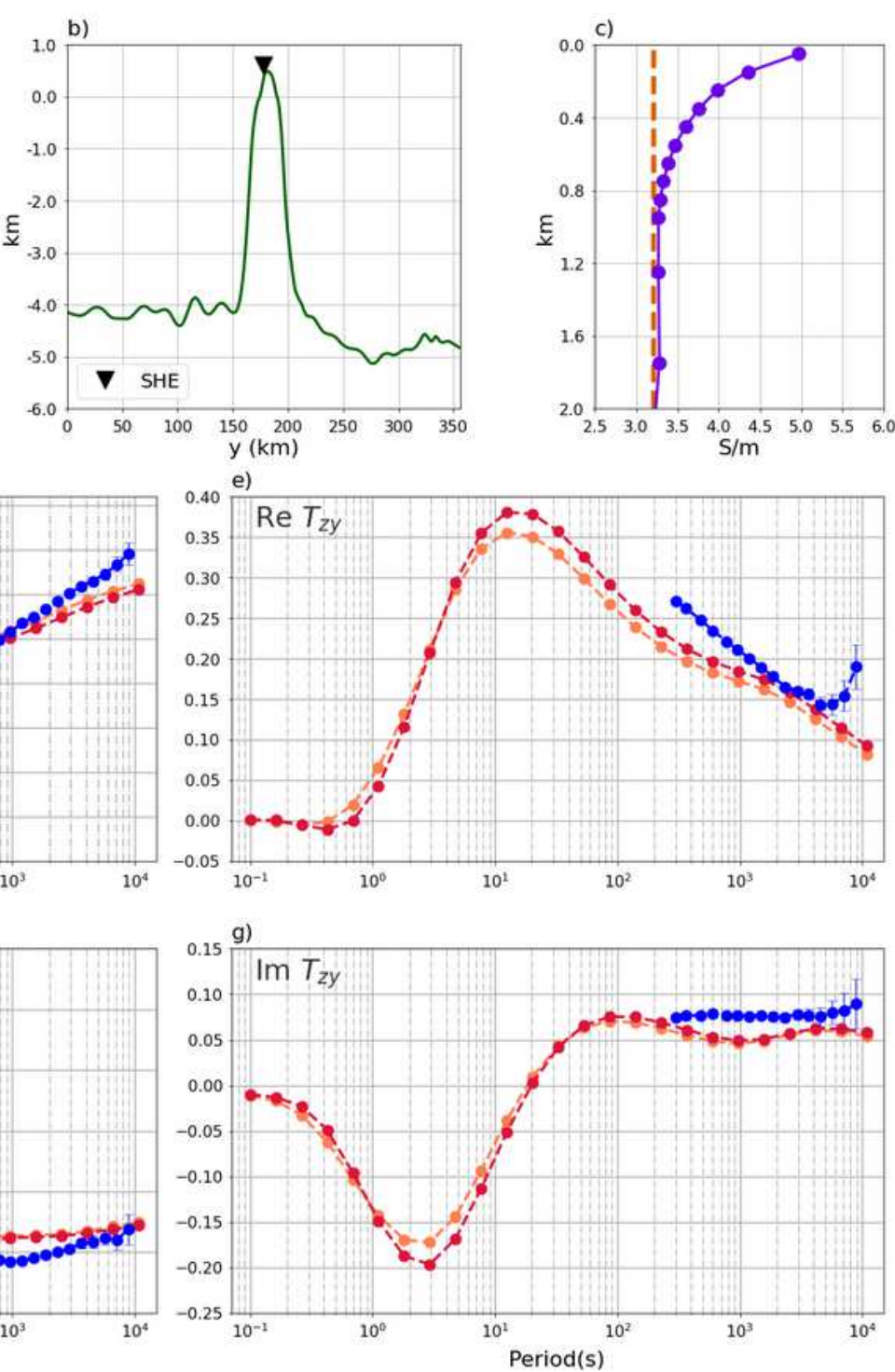

Period(s)

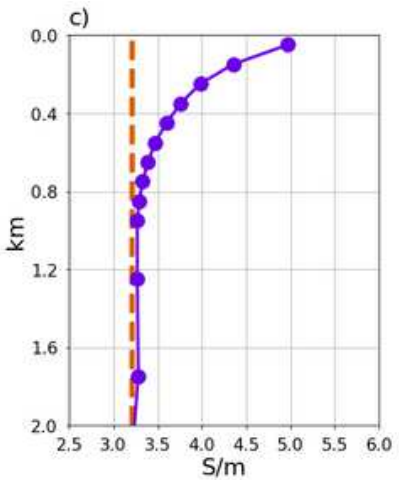

- Modelled with constant oceanic conductivity

- - Modelled with depth-varying oceanic conductivity

-๑- Observed
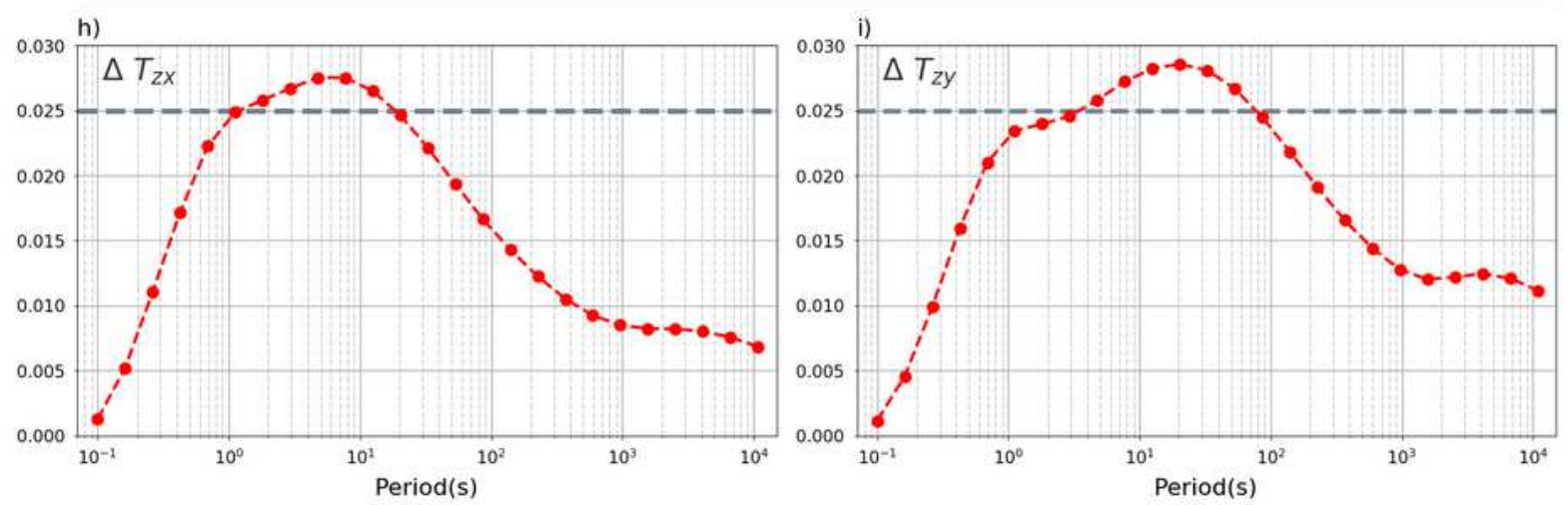

Figure 12

Same as Figure 5, but for St. Helena observatory (SHE). 

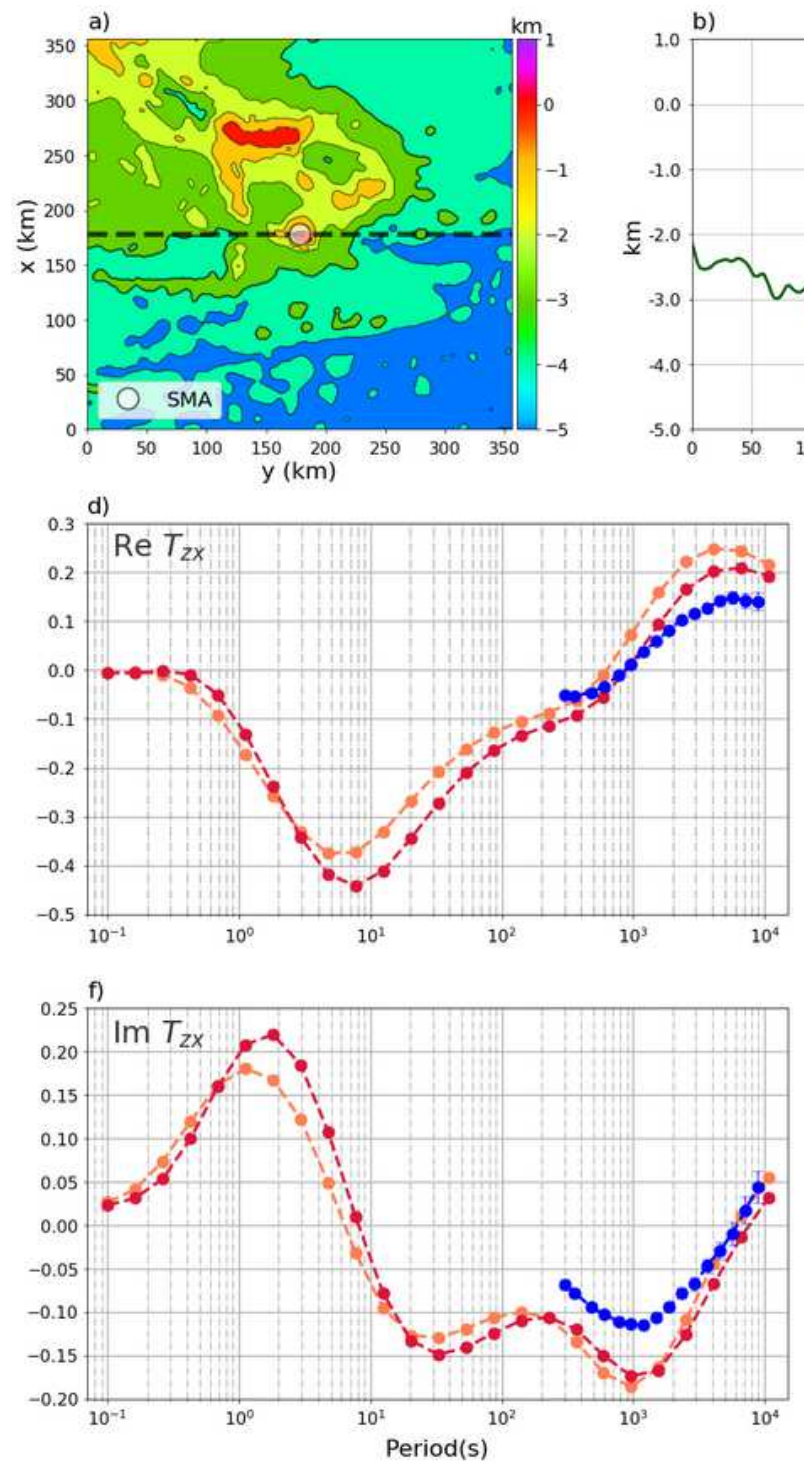

Period(s)

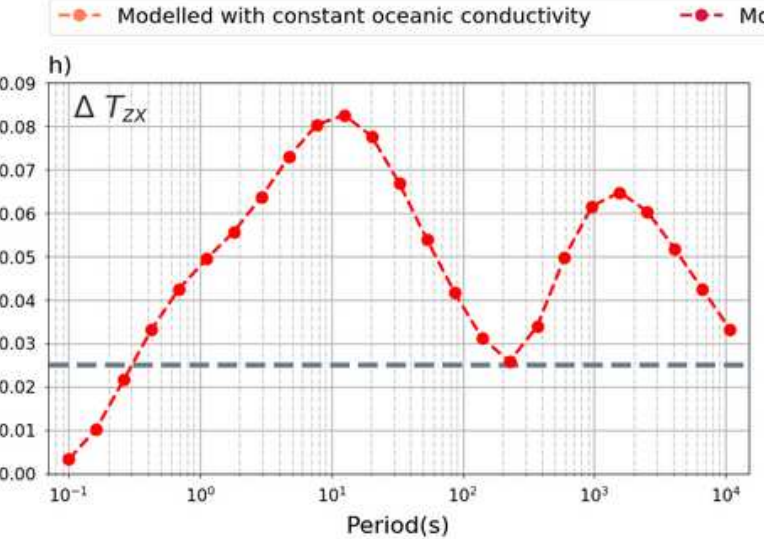

- Modelled with depth-varying oceanic conductivity
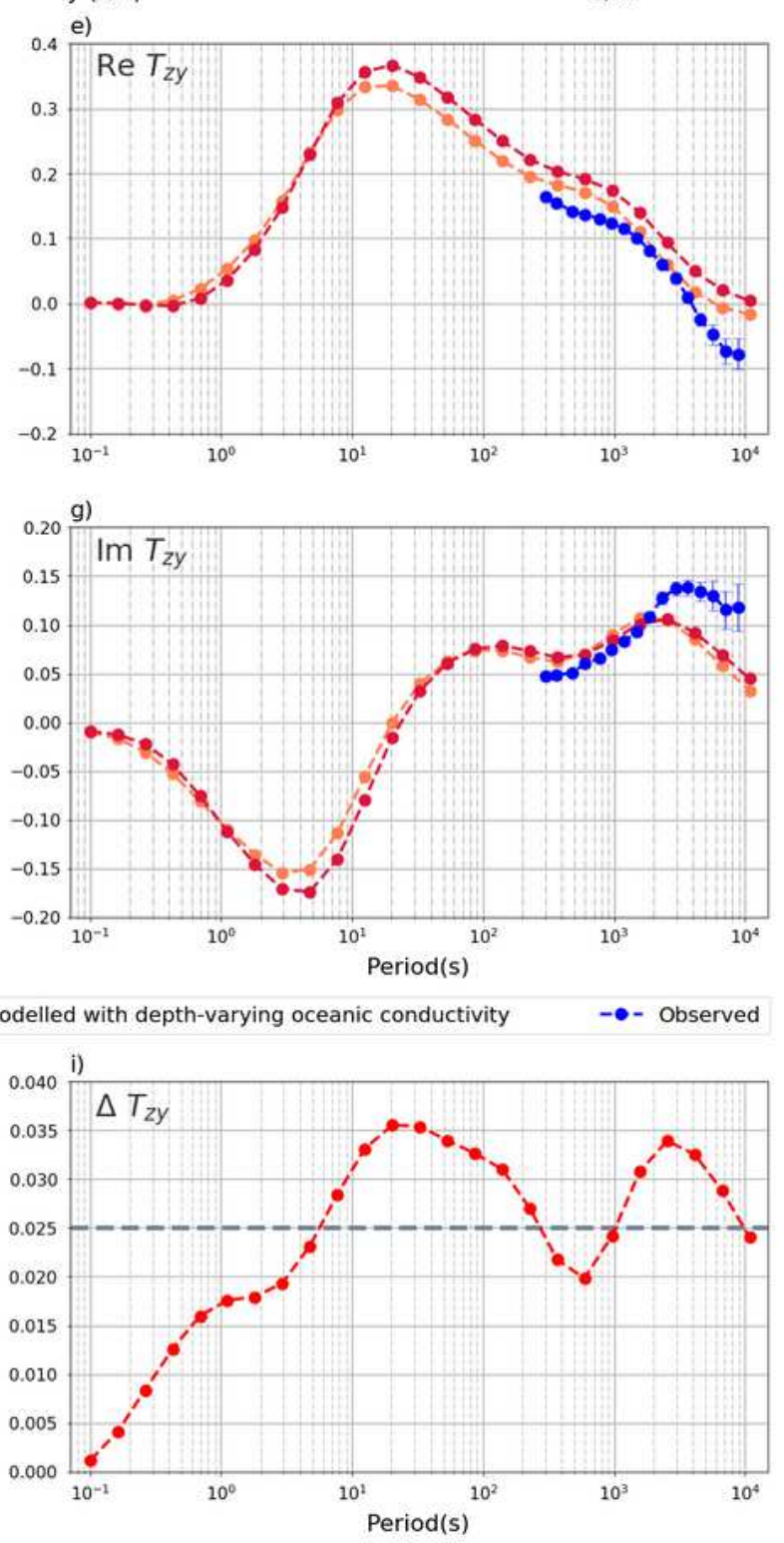

\section{Figure 13}

Same as Figure 5, but for Santa-Maria/Azores observatory (SMA). 

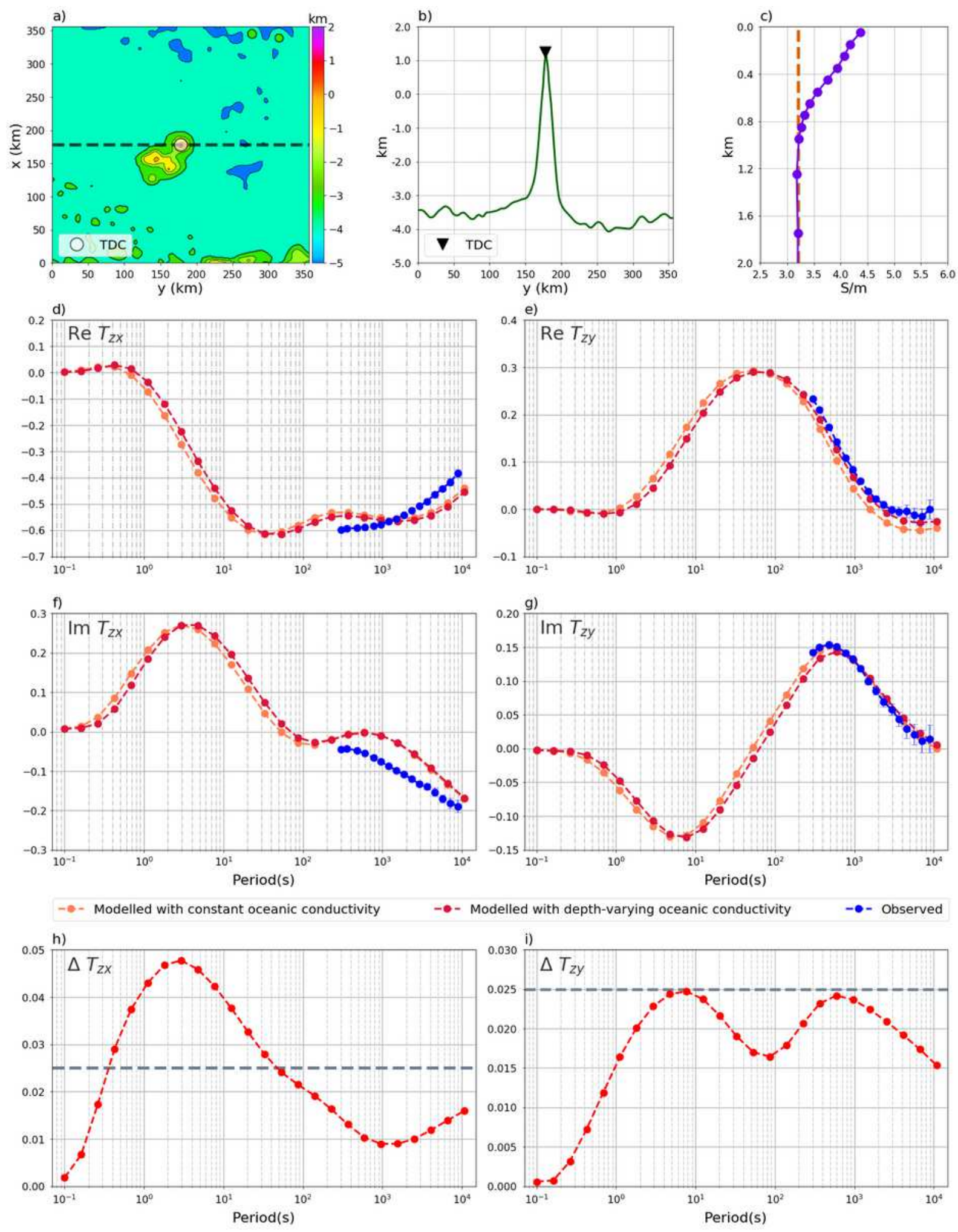

Modelled with depth-varying oceanic conductivity

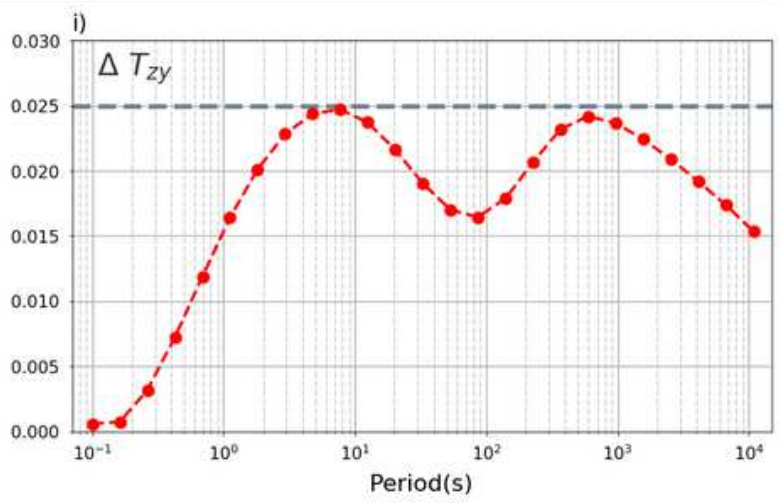

Figure 14

Same as Figure 5, but for Tristan da Cunha observatory (TDC). 

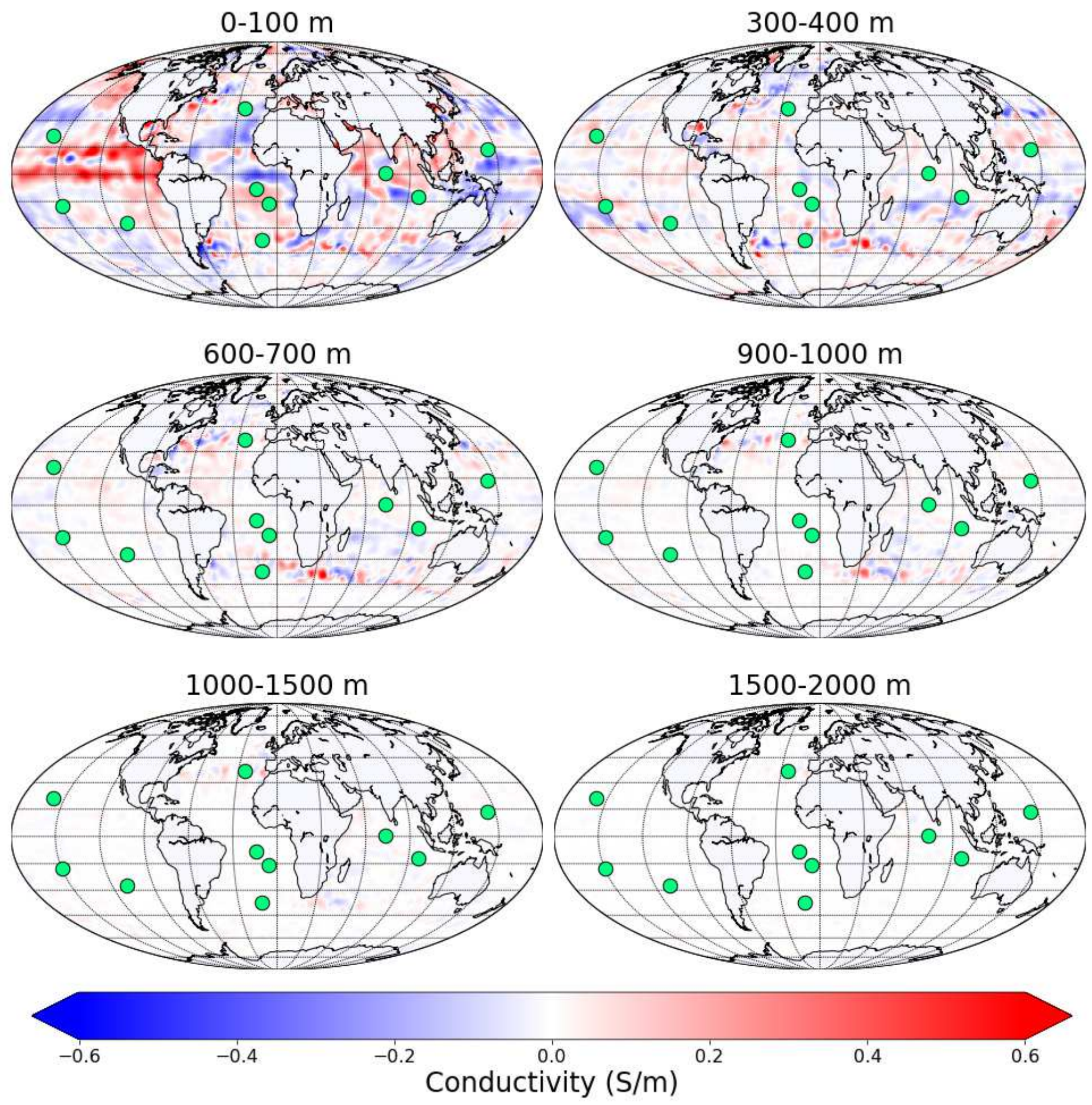

Figure 15

Global maps of difference between 2015 December and June oceanic conductivity models at six depth intervals. Green circles denote locations of geomagnetic observatories used in this study. 

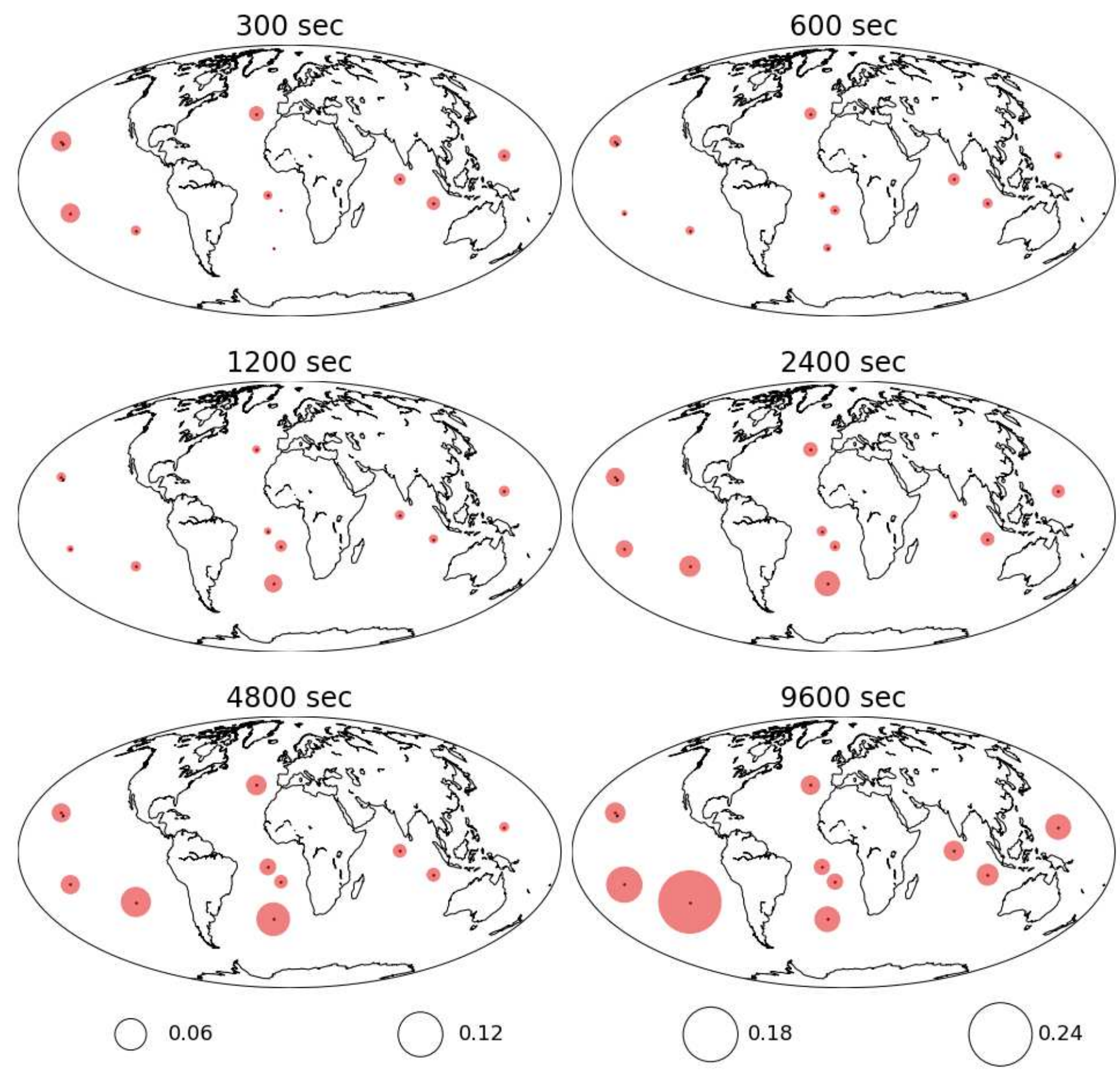

Figure 16

Difference between "December" and "June" Tzx at island observatories, shown as filled circles.

"Experimental" and "modeled" differences are colored by light and dark red, respectively. The size of circles below the plots indicates four ranges of differences. 

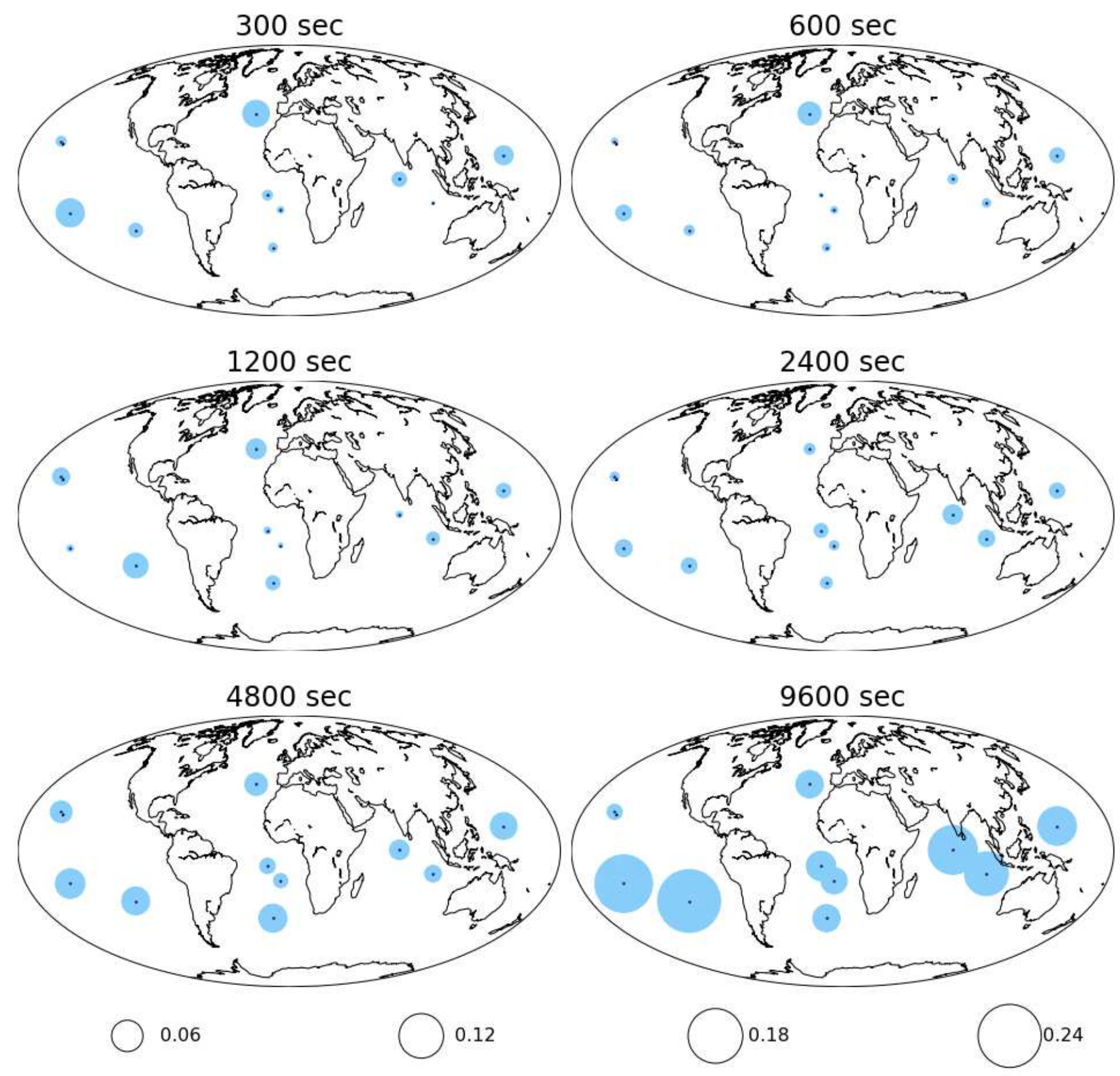

Figure 17

Same as Figure 16, but for Tzy.

\section{Supplementary Files}

This is a list of supplementary files associated with this preprint. Click to download.

- GASubmission.png 